TRANSACTIONS OF THE

AMERICAN MATHEMATICAL SOCIETY

Volume 364, Number 1, January 2012, Pages 447-491

S 0002-9947(2011)05452-8

Article electronically published on August 9, 2011

\title{
GROUND STATE AND NON-GROUND STATE SOLUTIONS OF SOME STRONGLY COUPLED ELLIPTIC SYSTEMS
}

\author{
DENIS BONHEURE, EDERSON MOREIRA DOS SANTOS, AND MIGUEL RAMOS
}

\begin{abstract}
We study an elliptic system of the form $L u=|v|^{p-1} v$ and $L v=$ $|u|^{q-1} u$ in $\Omega$ with homogeneous Dirichlet boundary condition, where $L u:=$ $-\Delta u$ in the case of a bounded domain and $L u:=-\Delta u+u$ in the cases of an exterior domain or the whole space $\mathbb{R}^{N}$. We analyze the existence, uniqueness, sign and radial symmetry of ground state solutions and also look for sign changing solutions of the system. More general non-linearities are also considered.
\end{abstract}

\section{Contents}

1. Introduction and statement of the results 448

2. The case of a bounded domain 452

3. The problem in the whole of $\mathbb{R}^{N} \quad 456$

4. The problem in the whole of $\mathbb{R}^{N}$ revisited 459

5. The case of an exterior domain $\quad 470$

5.1. Some technical estimates 471

5.2. Proof of Theorems 1.10 and $1.11 \quad 478$

Appendix A. Regularity and further estimates 481

A.1. Proof of Theorem $1.7 \quad 481$

A.2. Additional estimates 484

Acknowledgment $\quad 489$

References

Received by the editors October 16, 2009 and, in revised form, August 10, 2010.

2000 Mathematics Subject Classification. Primary 35J55; Secondary 35J50, 58E05.

Key words and phrases. Elliptic system, ground state solution, positive solution, sign changing solution, symmetry.

The first and second authors were partially supported by the bilateral agreement F.R.S.-FNRS \& CNPq.

The second author was supported by CAPES \# 4316/07-0 and FAPESP \# 07/54872-8.

The third author was supported by FCT, Fundação para a Ciência e a Tecnologia, Financiamento Base 2008 - ISFL/1/209.

(C)2011 American Mathematical Society Reverts to public domain 28 years from publication 


\section{INTRODUCTION AND STATEMENT OF THE RESULTS}

Let $\Omega$ be a smooth bounded domain in $\mathbb{R}^{N}$ with $N \geq 1$. We consider the system

$$
\left\{\begin{aligned}
-\Delta u & =|v|^{p-1} v & & \text { in } \Omega, \\
-\Delta v & =|u|^{q-1} u & & \text { in } \Omega \\
u, v & =0 & & \text { on } \partial \Omega
\end{aligned}\right.
$$

with $p>0$ and $q>0$.

One of the first results concerning positive solutions of (1.1) in the superlinear regime appeared in [9. The approach followed in this paper is based on topological arguments. In [16, an existence result is proved by use of variational arguments relying on a linking theorem. For the model case (1.1), this result applies if $p, q>$ 1 and satisfy the hypothesis (H1) described below. Since then, much effort has been devoted to the variational study of such elliptic systems leading to strongly indefinite functionals. We refer to the surveys [15, 32. It seems however that less attention has been paid to the existence of ground state solutions for strongly indefinite systems and their qualitative properties; see however [17, 33, 1]. This is partly due to the fact that simple tools available for analyzing a single equation cannot be used in a direct way to treat these systems.

In this paper, we are interested in the existence, positivity and uniqueness of ground state solutions for (1.1); in case $\Omega$ is a ball, we study their radial symmetry. We also analyze the cases where $\Omega$ is the whole space $\mathbb{R}^{N}$ or else an exterior domain of $\mathbb{R}^{N}$. We stress that the knowledge of the above-mentioned properties is important in our search of sign changing solutions for the system (1.6) below. We also believe that besides their own interests, the properties of the ground state solutions could be useful in other situations.

It is well known that the solution set of (1.1) is strongly affected by the values of the couple $(p, q)$. In the case when $N \geq 3$, as independently introduced by Mitidieri 25. and van der Vorst 37. (soon after considered by several authors, including Clément et al. 9] and Peletier-van der Vorst [27]), the so-called critical hyperbola plays a very important role. For instance, in the case of a smooth starshaped bounded domain $\Omega$, Mitidieri [25, 26] proved that (1.1) has no positive solution whenever

$$
\frac{1}{p+1}+\frac{1}{q+1} \leq 1-\frac{2}{N}
$$

On the other hand, in the case where

$$
p, q>0 \text { and } \frac{1}{p+1}+\frac{1}{q+1}>1-\frac{2}{N}
$$

is satisfied, then (1.1) can be treated variationally (assuming in addition $p, q>1$ ) as done in [16, 22] exploiting the strongly indefiniteness of the associated functional by means of a minimax method of Benci and Rabinowitz. Here we follow a different (more direct) approach which works fine under (H1). Indeed, see [19, Theorem 1.1] and Proposition 2.1 hereafter, it is known that (1.1) is equivalent to

$$
\left\{\begin{aligned}
\Delta\left(|\Delta u|^{\frac{1}{p}-1} \Delta u\right) & =|u|^{q-1} u & & \text { in } \Omega, \\
u=\Delta u & =0 & & \text { on } \partial \Omega,
\end{aligned}\right.
$$

in the sense that weak solutions of (1.2) correspond to classical solutions of (1.1). The idea of such a reduction goes back at least to P.-L. Lions 24]; see also [38, 23, 10, 11, 18. It turns out that (H1), the hypothesis for subcriticality for (1.1), is 
the right hypothesis to ensure a subcritical variational framework for dealing with (1.2).

Definition 1.1. Assume (H1). Let $E=W^{2, \frac{p+1}{p}}(\Omega) \cap W_{0}^{1, \frac{p+1}{p}}(\Omega)$ be endowed with the norm

$$
\|u\|=\left(\int_{\Omega}|\Delta u|^{\frac{p+1}{p}} d x\right)^{\frac{p}{p+1}}, u \in E .
$$

We say that $u \in E$ is a weak solution of (1.2) if

$$
\int_{\Omega}|\Delta u|^{\frac{1}{p}-1} \Delta u \Delta v d x=\int_{\Omega}|u|^{q-1} u v d x, \quad \forall v \in E .
$$

One easily checks that, under (H1), weak solutions of (1.2) are precisely the critical points of the $C^{1}(E, \mathbb{R})$ functional $J: E \rightarrow \mathbb{R}$ defined by

$$
J(u)=\frac{p}{p+1} \int_{\Omega}|\Delta u|^{\frac{p+1}{p}} d x-\frac{1}{q+1} \int_{\Omega}|u|^{q+1} d x .
$$

We make a preliminary remark in the case when $p q=1$. Let

$$
\lambda_{1, p}:=\inf \left\{\frac{\int_{\Omega}|\Delta u|^{\frac{p+1}{p}} d x}{\int_{\Omega}|u|^{\frac{p+1}{p}} d x}: u \in E \backslash\{0\}\right\} .
$$

Clearly, if $\lambda_{1, p}>1$, then (1.2) has no non-trivial weak solutions. Moreover, in general, $J(u)=0$ for any such weak solution $u \in E$; in particular, the value $J(u)$ does not distinguish weak solutions of (1.2) in the case when $p q=1$.

By virtue of this remark, and since we will be dealing with least energy solutions of (1.2), in the sequel we always assume that $p q \neq 1$. We now introduce the definition of a ground state solution. We anticipate that different equivalent choices are possible; see Propositions 2.1, 2.2, 3.1 and 4.7.

Definition 1.2. Assume (H1) and $p q \neq 1$. We say that $u \in E \backslash\{0\}$ is a ground state solution for (1.1) if $J$ a ttains its smallest non-zero critical value at $u$.

The next four theorems summarize the main results of this paper concerning (1.1). In connection with Theorem 1.5 below, we point out that (H1) holds in case $p q<1$ (recall that we always assume $p, q>0$ ).

Theorem 1.3. Assume (H1) and $p q \neq 1$. Then (1.1) has a ground state solution.

Theorem 1.4. Assume (H1) and $p q \neq 1$. Let $u \in E \backslash\{0\}$ be a ground state solution for (1.1) and let $v:=|\Delta u|^{\frac{1}{p}-1}(-\Delta u)$. Then $(u, v)$ is a classical solution for (1.1) and $u v>0$ in $\Omega$.

By analogy with the case of a single equation, one could think that the fact that any ground state solution is signed is somehow a trivial observation. It appears to be not so obvious for the ground state solutions of the system.

Theorem 1.5. Assume $p q<1$. Then (1.1) has, up to sign, a unique ground state solution $u$.

Theorem 1.6. Assume (H1), $p q \neq 1$ and $\Omega=B_{R}(0)$. Then (1.1) has, up to sign, a unique ground state solution $u$. Furthermore, by letting $u>0$ and $v:=$ $|\Delta u|^{\frac{1}{p}-1}(-\Delta u)>0$ we have that both $u$ and $v$ are radially symmetric and radially decreasing with respect to the origin. 
We mention that in [14, Theorem 1.2 (i)] the existence of a positive solution of (1.1) under hypotheses (H1) and $p q \neq 1$ is proved. Such a result is extended by combining Theorems 1.3 and 1.4 above. Also, we stress that our proof is much simpler. In order to prove Theorem 1.6, we extend some results in [20, Theorem 1], which deals with the case $p=1$. As for the uniqueness properties above, they turn out to be straightforward consequences of the results in [13, 14, once the remaining properties (positivity and symmetry) have been established.

Next we turn our attention to the system

$$
\left\{\begin{aligned}
-\Delta u+u=|v|^{p-1} v & \text { in } \mathbb{R}^{N} \\
-\Delta v+v=|u|^{q-1} u & \text { in } \mathbb{R}^{N}
\end{aligned}\right.
$$

and to the equation

$$
L\left(|L u|^{\frac{1}{p}-1} L u\right)=|u|^{q-1} u \text { in } \mathbb{R}^{N},
$$

where we denote $L u:=-\Delta u+u$.

Our results concerning (1.4) are similar to those stated for (1.1). However, here we have to restrict ourselves to the case when $p q>1$; this condition can be seen as a relaxed formulation of a superlinear behavior of the system. The main reason for this restriction is due to the following regularity result.

Theorem 1.7. Assume (H1) and $p q>1$. Let $u \in W^{2, \frac{p+1}{p}}\left(\mathbb{R}^{N}\right)$ be a weak solution of (1.5) and let $v:=|L u|^{\frac{1}{p}-1} L u$. Then $u \in W^{2, s}\left(\mathbb{R}^{N}\right)$ and $v \in W^{2, t}\left(\mathbb{R}^{N}\right)$ for all $s$ and $t$ in the range: $\max \left\{1, p^{-1}\right\}<s<\infty, \max \left\{1, q^{-1}\right\}<t<\infty$. Moreover, $u \in C^{2, \alpha}\left(\mathbb{R}^{N}\right)$ and $v \in C^{2, \beta}\left(\mathbb{R}^{N}\right)$ for all $\alpha$ and $\beta$ in the range: $0<\alpha \leq \min \{1, p\}$ and $0<\beta \leq \min \{1, q\}$, and $(u, v)$ is a classical solution for (1.4).

We stress that, even in the case $p, q>1$, Theorem 1.7 provides a sharper regularity result than the one in [33, Theorem 1]. The converse of Theorem 1.7 is given by Theorem 3.1

A word on the notation. In working with the system (1.4) we use the space $E=W^{2, \frac{p+1}{p}}\left(\mathbb{R}^{N}\right)$, equipped with the norm $\|u\|:=\left(\int_{\mathbb{R}^{N}}|-\Delta u+u|^{\frac{p+1}{p}} d x\right)^{\frac{p}{p+1}}$; it is known (see e.g. [21, Theorem 9.15 and Lemma 9.17]) that this norm is equivalent, on $E$, to the norm

$$
\left(\int_{R^{N}}\left|D^{2} u\right|^{\frac{p+1}{p}}+|\nabla u|^{\frac{p+1}{p}}+|u|^{\frac{p+1}{p}} d x\right)^{\frac{p}{p+1}} .
$$

As before, we say that $u \in E$ is a weak solution for (1.5) if

$$
\int_{\mathbb{R}^{N}}|-\Delta u+u|^{\frac{1}{p}-1}(-\Delta u+u)(-\Delta v+v) d x=\int_{\mathbb{R}^{N}}|u|^{q-1} u v d x, \quad \forall v \in E,
$$

that is, if $u$ is a critical point of the $C^{1}(E, \mathbb{R})$ functional

$$
J(u)=\frac{p}{p+1} \int_{\mathbb{R}^{N}}|-\Delta u+u|^{\frac{p+1}{p}} d x-\frac{1}{q+1} \int_{\mathbb{R}^{N}}|u|^{q+1} d x .
$$

Also, we say that $u \in E \backslash\{0\}$ is a ground state solution for (1.4) if $J$ attains its smallest non-zero critical value at $u$.

Theorem 1.8. Assume (H1) and $p q>1$. Then (1.4) has a ground state solution. 
Theorem 1.9. Assume (H1) and $p q>1$. Let $u \in E \backslash\{0\}$ be a ground state solution for (1.4) and let $v:=|L u|^{\frac{1}{p}-1}(L u)$. Then $(u, v)$ is a classical solution for (1.4) and $u v>0$ in $\mathbb{R}^{N}$.

We mention that in case $p(N-2), q(N-2)<(N+2)$ and $p, q>1$, then it follows from [17, Theorem 2.1] that solutions decay at infinity; as a consequence, according to [6. Theorem 2], in this case both $u$ and $v$ are radially symmetric and radially decreasing with respect to some point. We do not know whether this conclusion holds under the mere assumptions of Theorem 1.9. Indeed, when one of the powers is smaller than 1 , one cannot apply the moving plane method due to the lack of Lipschitz regularity.

At last, we consider the case of an exterior domain, namely

$$
\left\{\begin{aligned}
-\Delta u+u & =|v|^{p-1} v & & \text { in } \Omega, \\
-\Delta v+v & =|u|^{q-1} u & & \text { in } \Omega, \\
u, v & =0 & & \text { on } \partial \Omega,
\end{aligned}\right.
$$

with $\Omega=\mathbb{R}^{N} \backslash \omega$, where $\omega$ is a smooth and bounded domain of $\mathbb{R}^{N}, N \geq 3$. The notions of weak and ground state solutions are defined as above, by working in the natural space $E=W^{2, \frac{p+1}{p}}(\Omega) \cap W_{0}^{1, \frac{p+1}{p}}(\Omega)$ equipped with the norm $\|u\|:=$ $\left(\int_{\Omega}|L u|^{\frac{p+1}{p}} d x\right)^{\frac{p}{p+1}}$, with $L u:=-\Delta u+u$.

Following Cerami and Clapp [7, who deal with a single equation with homogeneous non-linearity, namely $-\Delta u+u=|u|^{p-1} u$ with $1<p<(N+2) /(N-2)$, we assume that $\Omega$ is invariant under the action of some closed subgroup $G$ of the group $O(N)$ of the orthogonal transformations in $\mathbb{R}^{N}, N \geq 3$. We denote by $G x:=\{g x: g \in G\}$ the orbit of $x$, by $\# G x$ its cardinality, and by $\ell=\ell(G):=$ $\min \left\{\# G x: x \in \mathbb{R}^{N} \backslash\{0\}\right\}$; we anticipate that in case $\ell=2$ we will exclude the $\mathbb{Z}_{2}$ symmetry. Accordingly, we define weak and ground state solutions by restricting the functional $J$ to the Sobolev space $E^{G}=W^{2, \frac{p+1}{p}}(\Omega)^{G} \cap W_{0}^{1, \frac{p+1}{p}}(\Omega)^{G}$ of $G$-invariant functions.

Theorem 1.10. Assume (H1) and $p, q>1$. Suppose moreover that $\ell>1$ and, in case $\ell=2$, that there exists a minimal orbit $G x$ such that $G x \neq\{x,-x\}$. Then (1.6) has a ground state solution, that is, a least energy $G$-invariant solution, with respect to the space $E^{G}$. Moreover, any such ground state solution $u$ is such that $u v>0$ in $\Omega$, where $v:=|L u|^{\frac{1}{p}-1}(L u)$.

A natural question arises whether the system admits further sign changing solutions, in the sense that both $u$ and $v$ change sign in $\Omega$. Again as in [7, this will be accomplished at the price of a further geometric hypothesis. We refer the reader to 7] for examples and a discussion on this assumption.

Theorem 1.11. Assume (H1) and $p, q>1$. Suppose moreover that $\ell>1$ and that $\mathbb{R}^{N} \backslash\{0\}$ contains a minimal $G$-orbit $G x$ with $|x|>\min \{|y-x|: y \in G x, y \neq x\}$. Then there exists a small $R_{0}>0$ such that if $\mathbb{R}^{N} \backslash B_{R_{0}}(0) \subset \Omega$, then (1.6) admits at least one $G$-invariant sign changing weak solution $(u, v)$.

The paper is organized as follows. Section 2 is devoted to the proof of Theorems 1.3 [1.6 and section 3 to the proof of Theorems 1.8 1.9. The latter results are 
partially extended in section 4 to general systems

$$
\left\{\begin{aligned}
-\Delta u+u & =g(v) \quad \text { in } \mathbb{R}^{N} \\
-\Delta v+v & =f(u) \quad \text { in } \mathbb{R}^{N}
\end{aligned}\right.
$$

under suitable assumptions on $f$ and $g$; see mainly Theorem 4.1. We have in mind examples such as $f(s) s=A|s|^{\alpha}+B|s|^{\beta}, g(s) s=A^{\prime}|s|^{\alpha^{\prime}}+B^{\prime}|s|^{\beta^{\prime}}$ with $A, B, A^{\prime}, B^{\prime}>0$ and $2<\beta \leq \alpha, 2<\beta^{\prime} \leq \alpha^{\prime}, 1 / \alpha+1 / \alpha^{\prime}>(N-2) / N$. The proofs are somehow more involved but they also serve as a preliminary step in the proof of Theorems 1.10 1.11. These are presented in section 5 and subsection $\mathrm{A} .2$ in the appendix. The proof of Theorem 1.7 is given in subsection A.1 of the appendix.

\section{The CASE OF A BOUNDED DOMAIN}

In this section we are mostly concerned with system (1.1) and the proofs of Theorems 1.3 1.6. In order to clarify our presentation and justify our previous definition of ground state solutions we begin with some regularity results.

Let us fix some notation and terminology. We consider the system

$$
\left\{\begin{aligned}
L u & =|v|^{p-1} v & & \text { in } \Omega \\
L v & =|u|^{q-1} u & & \text { in } \Omega \\
u, v & =0 & & \text { on } \partial \Omega
\end{aligned}\right.
$$

where either $L u=-\Delta u+u$ or $L u=-\Delta u$. We assume (H1) holds and we say that $(u, v)$ is a strong solution for (2.1) if $u \in W^{2, \frac{p+1}{p}}(\Omega) \cap W_{0}^{1, \frac{p+1}{p}}(\Omega), v \in W^{2, \frac{q+1}{q}}(\Omega) \cap$ $W_{0}^{1, \frac{q+1}{q}}(\Omega)$ and $(u, v)$ satisfies (2.1) a.e. in $\Omega$. We also consider the problem

$$
\left\{\begin{aligned}
L\left(|L u|^{\frac{1}{p}-1} L u\right) & =|u|^{q-1} u & & \text { in } \Omega, \\
u=\Delta u & =0 & & \text { on } \partial \Omega .
\end{aligned}\right.
$$

We define the weak solutions for $(2.2)$ as the critical points of the $C^{1}(E, \mathbb{R})$ functional

$$
J(u)=\frac{p}{p+1} \int_{\Omega}|L u|^{\frac{p+1}{p}} d x-\frac{1}{q+1} \int_{\Omega}|u|^{q+1} d x, \quad u \in E,
$$

where $E=W^{2, \frac{p+1}{p}}(\Omega) \cap W_{0}^{1, \frac{p+1}{p}}(\Omega)$, endowed with the norm defined by

$$
\|u\|=\left(\int_{\Omega}|L u|^{\frac{p+1}{p}} d x\right)^{\frac{p}{p+1}}
$$

In case $p(N-2), q(N-2)<N+2$, we define the weak solutions for (2.1) as the critical points of the $C^{1}\left(H_{0}^{1}(\Omega) \times H_{0}^{1}(\Omega), \mathbb{R}\right)$ functional

$$
I(u, v)=\langle u, v\rangle-\int_{\Omega}\left(\frac{|u|^{q+1}}{q+1}+\frac{|v|^{p+1}}{p+1}\right) d x, \quad u, v \in H_{0}^{1}(\Omega),
$$

where $\langle u, v\rangle:=\int_{\Omega}(\langle\nabla u, \nabla v\rangle+u v) d x$ if $L u=-\Delta u+u$ and $\langle u, v\rangle:=\int_{\Omega}\langle\nabla u, \nabla v\rangle d x$ if $L u=-\Delta u$.

Proposition 2.1. Assume (H1) holds. Let $u \in E$ and set $v:=|L u|^{\frac{1}{p}-1} L u$. The following statements are equivalent:

(i) $u$ is a critical point of $J$.

(ii) $(u, v)$ is a strong solution of (2.1).

(iii) $u, v \in W^{2, s}(\Omega)$ for all $1 \leq s<\infty$ and $(u, v)$ is a strong solution of (2.1).

(iv) $u, v \in C^{2}(\bar{\Omega}) \cap C_{0}(\bar{\Omega})$ and $(u, v)$ is a classical solution of (2.1). 
(v) $u \in C^{2, \alpha}(\bar{\Omega})$ and $v \in C^{2, \beta}(\bar{\Omega})$ for all $\alpha$ and $\beta$ in the range: $0<\alpha \leq \min \{1, p\}$ and $0<\beta \leq \min \{1, q\}$, and $(u, v)$ is a classical solution of (2.1).

In any such case, we have that $J(u)=I(u, v)$.

Proposition 2.2. Assume $p(N-2), q(N-2)<N+2$. Let $u, v \in H_{0}^{1}(\Omega)$. The following statements are equivalent:

(i) $(u, v)$ is a critical point of $I$.

(ii) $u$ is a critical point of $J$ and $v=|L u|^{\frac{1}{p}-1} L u$.

In any such case, we have that $J(u)=I(u, v)$.

The proofs for Propositions 2.1 and 2.2 are similar, though easier, to the proof of Theorem 1.7 see subsection A.1. The identity $J(u)=I(u, v)$ is obtained in a straightforward way; see also (4.9) hereafter.

In the remainder of this section we will be working on the system (1.1) or on the equation (1.2).

In the sequel we assume that (H1) holds and $p q \neq 1$. We denote by $\mathcal{N}_{J}$ the Nehari manifold associated to the functional $J$, namely

$$
\mathcal{N}_{J}:=\left\{u \in E \backslash\{0\}: J^{\prime}(u) u=0\right\},
$$

and introduce the minimization problems

$$
c_{J}:=\inf _{u \in \mathcal{N}_{J}} J(u)
$$

and

$$
\alpha_{p, q}:=\inf \left\{\int_{\Omega}|\Delta u|^{\frac{p+1}{p}} d x: u \in E,|u|_{q+1}^{q+1}=1\right\},
$$

where we denote $|u|_{q+1}^{q+1}:=\int_{\Omega}|u|^{q+1}$. Note that if it is achieved, $1 /\left(\alpha_{p, q}\right)^{p /(p+1)}$ is the optimal constant for the embedding of $E$ into $L^{q+1}(\Omega)$.

We start by observing that given $u \in E \backslash\{0\}$ there exists a unique $t=t(u)>0$ such that $t(u) u \in \mathcal{N}_{J}$, which is explicitly given by

$$
t(u)=\left(\frac{\|u\|^{\frac{p+1}{p}}}{|u|_{q+1}^{q+1}}\right)^{\frac{p}{p q-1}} .
$$

Now, let $u \in \mathcal{N}_{J}$. Then $0=\left\langle J^{\prime}(u), u\right\rangle=\|u\|^{\frac{p+1}{p}}-|u|_{q+1}^{q+1}$, and therefore

$$
J(u)=\frac{p}{p+1}\|u\|^{\frac{p+1}{p}}-\frac{1}{q+1}|u|_{q+1}^{q+1}=\frac{p q-1}{(p+1)(q+1)}\|u\|^{\frac{p+1}{p}} .
$$

Furthermore,

$$
\frac{\|u\|^{\frac{p+1}{p}}}{|u|_{q+1}^{\frac{p+1}{p}}}=\frac{\|u\|^{\frac{p+1}{p}}}{\|u\|^{\left(\frac{p+1}{p}\right)^{2} \frac{1}{q+1}}}=\left(\frac{(p+1)(q+1)}{p q-1} J(u)\right)^{\frac{p q-1}{p(q+1)}} .
$$

Lemma 2.3. The minimization problems (2.3) and (2.4) are equivalent in the sense that:

(i) Given a minimizing sequence $\left(u_{n}\right) \subset \mathcal{N}_{J}$ for (2.3), $\left(\left|u_{n}\right|_{q+1}^{-1} u_{n}\right)$ is a minimizing sequence for (2.4).

(ii) Given a minimizing sequence $\left(\bar{u}_{n}\right)$ for (2.4),$\left(\left\|\bar{u}_{n}\right\|^{\frac{p+1}{p q-1}} \bar{u}_{n}\right) \subset \mathcal{N}_{J}$ is a minimizing sequence for (2.3). 
(iii) We have the equality

$$
c_{J}=\frac{p q-1}{(p+1)(q+1)} \alpha_{p, q}^{\frac{p(q+1)}{p q-1}} .
$$

(iv) The optimal constant $\alpha_{p, q}$ is attained if and only if $c_{J}$ is attained. In addition, if $\bar{u}$ is a solution for (2.4), then $\|\bar{u}\|^{\frac{p+1}{p q-1}} \bar{u}=\alpha_{p, q}^{\frac{p}{p q-1}} \bar{u}$ is a solution for (2.3). Conversely, if $u$ is a solution for (2.3), then $|u|_{q+1}^{-1} u$ is a solution for (2.4).

Proof. Let $\left(u_{n}\right) \subset \mathcal{N}_{J}$ be a minimizing sequence for (2.3). Then, by (2.7),

$$
\begin{aligned}
\alpha_{p, q} \leq \lim _{n \rightarrow \infty} \frac{\left\|u_{n}\right\|^{\frac{p+1}{p}}}{\left|u_{n}\right|_{q+1}^{\frac{p+1}{p}}}=\lim _{n \rightarrow \infty}\left(\frac{(p+1)(q+1)}{p q-1}\right. & \left.J\left(u_{n}\right)\right)^{\frac{p q-1}{p(q+1)}} \\
& =\left(\frac{(p+1)(q+1)}{p q-1} c_{J}\right)^{\frac{p q-1}{p(q+1)}} .
\end{aligned}
$$

On the other hand, let $\left(\bar{u}_{n}\right)$ be a minimizing sequence for (2.4). Then, by (2.5), $\left(\left\|\bar{u}_{n}\right\|^{\frac{p+1}{p q-1}} \bar{u}_{n}\right) \subset \mathcal{N}_{J}$ and so, by (2.6),

$$
\begin{aligned}
c_{J} \leq \lim _{n \rightarrow \infty} J\left(\left\|\bar{u}_{n}\right\|^{\frac{p+1}{p q-1}} \bar{u}_{n}\right)=\frac{p q-1}{(p+1)(q+1)} \lim _{n \rightarrow \infty} & \left\|\bar{u}_{n}\right\|^{\frac{p(q+1)}{p q-1} \frac{p+1}{p}} \\
& =\frac{p q-1}{(p+1)(q+1)} \alpha_{p, q}^{\frac{p(q+1)}{p q-1}} .
\end{aligned}
$$

The proof for (i)-(iii) follows from (2.9)-(2.10).

Now, suppose that $\bar{u} \in E$ is such that $|\bar{u}|_{q+1}=1$ and $\alpha_{p, q}=\|\bar{u}\|^{\frac{p+1}{p}}$. Then, by (2.5), $\|\bar{u}\|^{\frac{p+1}{p q-1}} \bar{u}=\alpha_{p, q}^{\frac{p}{p q-1}} \bar{u} \in \mathcal{N}_{J}$. Furthermore, for every $u \in \mathcal{N}_{J}$, we see from (2.7) that

$$
\begin{aligned}
\left(\frac{(p+1)(q+1)}{p q-1} J\left(\alpha_{p, q}^{\frac{p}{p q-1}} \bar{u}\right)\right)^{\frac{p q-1}{p(q+1)}} & =\alpha_{p, q} \\
& \leq \frac{\|u\|^{\frac{p+1}{p}}}{|u|_{q+1}^{\frac{p+1}{p}}}=\left(\frac{(p+1)(q+1)}{p q-1} J(u)\right)^{\frac{p q-1}{p(q+1)}}
\end{aligned}
$$

that is, $J\left(\alpha_{p, q}^{\frac{p}{p q-1}} \bar{u}\right) \leq J(u)$. Therefore, $J\left(\alpha_{p, q}^{\frac{p}{p q-1}} \bar{u}\right)=c_{J}$.

Conversely, suppose that $u \in \mathcal{N}_{J}$ is such that $J(u)=c_{J}$. Then, by (2.7) and (2.8),

$$
\frac{\|u\|^{\frac{p+1}{p}}}{|u|_{q+1}^{\frac{p+1}{p}}}=\left(\frac{(p+1)(q+1)}{p q-1} J(u)\right)^{\frac{p q-1}{p(q+1)}}=\left(\frac{(p+1)(q+1)}{p q-1} c_{J}\right)^{\frac{p q-1}{p(q+1)}}=\alpha_{p, q} .
$$

This completes the proof of (iv).

Lemma 2.4. The optimal constant $\alpha_{p, q}$ is attained; i.e., there exists $u \in E$ such that $|u|_{q+1}=1$ and $\|u\|^{\frac{p+1}{p}}=\alpha_{p, q}$.

Proof. It is a straightforward consequence of the fact that $E$ is compactly embedded into $L^{q+1}(\Omega)$, since $\Omega$ is a bounded smooth domain.

Our next lemma shows that the minimization problem (2.3) is a natural method for finding ground state solutions for (1.1). 
Lemma 2.5. If $u \in \mathcal{N}_{J}$ is such that $J(u)=c_{J}$, then $u$ is a ground state solution for (1.1). Conversely, if $u$ is a ground state solution for (1.1), then $J(u)=c_{J}$.

Proof. Let $G(u)=J^{\prime}(u) u$. Then $G^{\prime}(u) u=\frac{1-p q}{p}\|u\|^{\frac{p+1}{p}} \neq 0$ for every $u \in \mathcal{N}_{J}$, and the first conclusion follows from the Lagrange multiplier theorem. In particular, thanks also to Lemmas 2.3 (iv) and 2.4, we have that there exists $\bar{u} \in \mathcal{N}_{J}$ such that $J(\bar{u})=c_{J}$ and $J^{\prime}(\bar{u})=0$, and this yields our second conclusion.

Lemma 2.6. Let $u \in \mathcal{N}_{J}$ be such that $J(u)=c_{J}$. Then $u,-\Delta u>0$ in $\Omega$, or else $u,-\Delta u<0$ in $\Omega$.

Proof. We infer from Lemma 2.5 and Proposition 2.1 that the couple $(u, v)$ with $v:=|\Delta u|^{\frac{1}{p}-1}(-\Delta u)$ classically solves the problem (1.1) and we have that $u, v \in$ $C^{2, \alpha}(\bar{\Omega})$ for a suitable $\alpha \in(0,1)$. By using the strong maximum principle, we will be done if we show that $-\Delta u$ does not change sign in $\Omega$.

Now, we use an argument that goes back at least to van der Vorst 36. Namely, let $w \in E$ be such that $-\Delta w=|\Delta u|$, so that $-\Delta(w \pm u) \geq 0$. Arguing by contradiction, suppose that $-\Delta u$ does change sign in $\Omega$. Then $-\Delta(w \pm u) \neq 0$ and the strong maximum principle implies that $w>|u|$. Then, also using Lemma 2.3 (iv), we have that

$$
\int_{\Omega}\left|\Delta\left(\frac{w}{|w|_{q+1}}\right)\right|^{\frac{p+1}{p}} d x=\int_{\Omega}\left(\frac{|\Delta u|^{\frac{p+1}{p}}}{|w|_{q+1}^{\frac{p+1}{p}}}\right) d x<\int_{\Omega}\left(\frac{|\Delta u|^{\frac{p+1}{p}}}{|u|_{q+1}^{\frac{p+1}{p}}}\right) d x=\alpha_{p, q} .
$$

This contradicts the definition of $\alpha_{p, q}$ and completes the proof.

Remark 2.7. Lemma 2.6 implies that the mountain-pass level of $J$ is achieved by a function $u$ satisfying $u,-\Delta u>0$ or $u,-\Delta u<0$. In [11, in order to find a positive critical point of $J$, the authors consider the functional $J^{+}$defined by

$$
J^{+}(u)=\frac{p}{p+1} \int_{\Omega}|\Delta u|^{\frac{p+1}{p}} d x-\frac{1}{q+1} \int_{\Omega}\left|u^{+}\right|^{q+1} d x .
$$

Indeed, using a clever trick, the authors prove that every critical point of $J^{+}$satisfies $u,-\Delta u>0$ so that it is also a critical point of $J$. A direct consequence of Lemma 2.6 is that the mountain-pass levels of $J$ and $J^{+}$coincide.

We denote by $B_{R}$ the open ball in $\mathbb{R}^{N}$ of radius $R$ centered at the origin and, for a given function $f \in C\left(\overline{B_{R}}\right), f \geq 0$, we denote by $f^{*}$ the Schwarz symmetric function associated to $f$, namely the radially symmetric, radially non-increasing function, equi-measurable with $f$. We next recall a result on the properties of the Schwarz symmetrization. The first conclusion in the lemma below can be found in [34, Theorem 1] and the second one is a particular case of [3, Theorem 1].

Lemma 2.8. Let $f \in C\left(\overline{B_{R}}\right), f \geq 0$, and $u, w$ satisfy

$$
\left\{\begin{array} { r l l } 
{ - \Delta u } & { = f } & { \text { in } B _ { R } , } \\
{ u } & { = } & { \text { on } \partial B _ { R } , }
\end{array} \quad \left\{\begin{array}{rlll}
-\Delta w & = & f^{*} & \text { in } B_{R}, \\
w & = & 0 & \text { on } \partial B_{R} .
\end{array}\right.\right.
$$

Then $u^{*} \leq w$ in $B_{R}$. Furthermore,

$$
\left|u^{*}<w\right|=0 \text { if and only if } f=f^{*} .
$$


Proof of Theorems 1.3 1.6. The conclusion in Theorem 1.3 follows from Lemma 2.3 (iv), Lemma 2.4 and Lemma 2.5. Thanks also to Lemma 2.6. we have proved Theorem 1.4. The uniqueness property of Theorem 1.5 is then a direct consequence of [13, Theorem 3]. As for Theorem [1.6, once the radial symmetry is established, the uniqueness of the ground state follows from [14. Theorem 1.1 (i)]. Now, let $u \in E$ be a ground state solution for (1.1) such that $u,-\Delta u>0$ in $\Omega$, and set $f:=-\Delta u \in C\left(\overline{B_{R}}\right)$. Let $w$ be such that $-\Delta w=f^{*}$ in $B_{R}, w=0$ on $\partial B_{R}$. In order to complete our proof we must show that $f=f^{*}$. Arguing by contradiction, suppose $f \neq f^{*}$. It follows then from Lemma 2.8 that $|w|_{q+1}>\left|u^{*}\right|_{q+1}$. Thus, also using Lemma 2.3 (iv), we have that

$$
\begin{aligned}
\int_{B_{R}}\left|\Delta\left(\frac{w}{|w|_{q+1}}\right)\right|^{\frac{p+1}{p}} & d x=\int_{B_{R}}\left(\frac{|\Delta u|^{\frac{p+1}{p}}}{|w|_{q+1}^{\frac{p+1}{p}}}\right) d x \\
& <\int_{B_{R}}\left(\frac{|\Delta u|^{\frac{p+1}{p}}}{\left|u^{*}\right|_{q+1}^{\frac{p+1}{p}}}\right) d x=\int_{B_{R}}\left|\Delta\left(\frac{u}{|u|_{q+1}}\right)\right|^{\frac{p+1}{p}} d x=\alpha_{p, q} .
\end{aligned}
$$

This contradicts the definition of $\alpha_{p, q}$ and completes the argument.

Remark 2.9. The fact that the ground state solutions $(u, v)$ of (1.1) with $\Omega=$ $B_{R}(0)$ are radially symmetric and radially decreasing with respect to the origin can be deduced from a more general result of Troy [35, based on the moving plane method, once it is known that $u$ and $v$ are positive. However, the approach based on symmetrization techniques is more direct and natural for ground state solutions of (1.1).

\section{The PRoblem in the Whole of $\mathbb{R}^{N}$}

This section is devoted to the proof of Theorems 1.8 and 1.9. Unless otherwise stated, we assume that (H1) holds and $p q>1$. As in the previous section, we start with some regularity results, namely a converse of Theorem 1.7 together with some extra information.

Proposition 3.1. Assume (H1) and $p q>1$. Let $(u, v)$ be a strong solution of (1.4). Then $u, v$ are as regular as in Theorem 1.7, $u$ is a weak solution of (1.5), and $J(u)=I(u, v)$.

Proposition 3.2. Assume $p, q>1$ and $p(N-2), q(N-2)<N+2$. Let $u, v \in$ $H^{1}\left(\mathbb{R}^{N}\right)$. The following statements are equivalent:

(i) $(u, v)$ is a critical point of $I$.

(ii) $u$ is a critical point of $J$ and $v=|L u|^{\frac{1}{p}-1} L u$. In any such case, $I(u, v)=$ $J(u)$.

The proofs for Propositions 3.1 and 3.2 are similar to the proof of Theorem 1.7 The identity $I(u, v)=J(u)$ is obtained in a straightforward way; see also (4.9). We mention that by combining Proposition 3.2 with [17, Theorem 2.2], we deduce that, in case $p, q>1$ and $p(N-2), q(N-2)<N+2$, the critical points of $I$ decay exponentially at infinity. 
As in the previous section, we introduce the Nehari manifold $\mathcal{N}_{J}$ associated to $J, c_{J}:=\inf _{\mathcal{N}_{J}} J$ and

$$
\alpha_{p, q}:=\inf \left\{\int_{\mathbb{R}^{N}}|L u|^{\frac{p+1}{p}} d x: u \in E,|u|_{q+1}^{q+1}=1\right\}
$$

where we denote $L u:=-\Delta u+u$. It is clear that statements similar to Lemma 2.3 and Lemma 2.5 can be formulated in this setting.

Lemma 3.3. Let $\Omega \subset \mathbb{R}^{N}$ be a smooth bounded domain and let $B$ be the open ball of $\mathbb{R}^{N}$ centered at the origin such that $|B|=|\Omega|$. Let $p, q>0$ be such that $\frac{1}{p+1}+\frac{1}{q+1} \geq 1-\frac{2}{N}$. Let $f \in L^{\frac{p+1}{p}}(\Omega)$ and let $u$ and $w$ be the strong solutions of

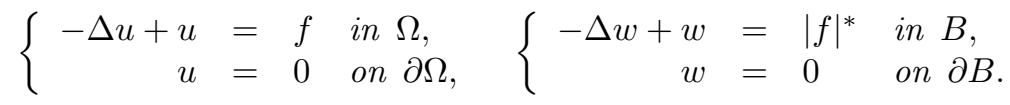

Then

$$
\int_{\Omega}|u|^{q+1} d x \leq \int_{B}|w|^{q+1} d x .
$$

Proof. In case $f$ is smooth this is inequality (9) in 2. In the general case one can use a density argument based on the continuity of the Schwarz symmetrization $*: L^{\frac{p+1}{p}}(\Omega) \rightarrow L^{\frac{p+1}{p}}(B)$ (see [8, 40]) and the continuous embeddings $W^{2, \frac{p+1}{p}}(\Omega) \hookrightarrow$ $L^{q+1}(\Omega), W^{2, \frac{p+1}{p}}(B) \hookrightarrow L^{q+1}(B)$.

Lemma 3.4. Let $p, q>0$ be such that $p q \geq 1$ and $\frac{1}{p+1}+\frac{1}{q+1} \geq 1-\frac{2}{N}$. Let $f \in L^{\frac{p+1}{p}}\left(\mathbb{R}^{N}\right)$ and let $u$ and $\bar{u}$ be the strong solutions of

$$
-\Delta u+u=f, \quad-\Delta \bar{u}+\bar{u}=|f|^{*} \text { in } \mathbb{R}^{N} .
$$

Then

$$
\int_{\mathbb{R}^{N}}|u|^{q+1} d x \leq \int_{\mathbb{R}^{N}}|\bar{u}|^{q+1} d x
$$

Proof. We define $f_{n}:=\left.f\right|_{B_{n}}$, where $B_{n}$ stands for the open ball in $\mathbb{R}^{N}$ with center 0 and radius $n$. We denote by $\widetilde{h}$ the extension by zero of a given function $h: B_{n} \rightarrow \mathbb{R}$. With this notation, it is easy to see that

$$
\widetilde{\left|f_{n}\right|^{*}}=\widetilde{\left|f_{n}\right|^{*}}{ }^{*}=\left|\widetilde{f_{n}}\right|^{*} \text { in } \mathbb{R}^{N} \text {. }
$$

Indeed, the first identity holds true because $\widetilde{\left|f_{n}\right|^{*}}$ is radially symmetric and radially decreasing with respect to the origin, while the second one holds since $\widetilde{\left|f_{n}\right|^{*}}$ and $\left|\widetilde{f_{n}}\right|$ share the same distribution function. We observe that we have used the same notation $*$ to represent both Schwarz symmetrizations in $\mathbb{R}^{N}$ and in $B_{n}$.

Now, for each $n \in \mathbb{N}$, let $u_{n}, \bar{u}_{n}$ and $w_{n}$ be the strong solutions of

$$
\left\{\begin{array} { r l l } 
{ - \Delta u _ { n } + u _ { n } = f _ { n } } & { \text { in } B _ { n } , } \\
{ u _ { n } = 0 } & { \text { on } \partial B _ { n } , }
\end{array} \quad \left\{\begin{array}{rl}
-\Delta w_{n}+w_{n}=\left|f_{n}\right|^{*} & \text { in } B_{n}, \\
w_{n}=0 & \text { on } \partial B_{n},
\end{array}\right.\right.
$$

and

$$
-\Delta \bar{u}_{n}+\bar{u}_{n}=\widetilde{\left|f_{n}\right|^{*}} \text { in } \mathbb{R}^{N}
$$


It follows from the maximum principle that $\bar{u}_{n} \geq w_{n}$ a.e. in $B_{n}$. Combining this with Lemma 3.3 yields

$$
\int_{B_{n}}\left|u_{n}\right|^{q+1} d x \leq \int_{B_{n}}\left|w_{n}\right|^{q+1} d x \leq \int_{\mathbb{R}^{N}}\left|\bar{u}_{n}\right|^{q+1} d x, \quad \forall n \in \mathbb{N} .
$$

By the continuity of the Schwarz symmetrization $*: L^{\frac{p+1}{p}}\left(\mathbb{R}^{N}\right) \rightarrow L^{\frac{p+1}{p}}\left(\mathbb{R}^{N}\right)$ and since $\widetilde{f_{n}} \rightarrow f$ in $L^{\frac{p+1}{p}}\left(\mathbb{R}^{N}\right)$, it follows from (3.2) that $\bar{u}_{n} \rightarrow \bar{u}$ in $W^{2, \frac{p+1}{p}}\left(\mathbb{R}^{N}\right)$. In particular,

$$
\lim _{n \rightarrow \infty} \int_{\mathbb{R}^{N}}\left|\bar{u}_{n}\right|^{q+1} d x=\lim _{n \rightarrow \infty} \int_{\mathbb{R}^{N}}|\bar{u}|^{q+1} d x .
$$

Now, it is clear that $\widetilde{u_{n}} \rightarrow u$ weakly in $L^{q+1}\left(\mathbb{R}^{N}\right)$. Indeed, we have that

$$
\lim _{n \rightarrow \infty} \int_{\mathbb{R}^{N}}\left(u-\widetilde{u_{n}}\right)(-\Delta \varphi+\varphi) d x=0, \quad \forall \varphi \in \mathcal{D}\left(\mathbb{R}^{N}\right),
$$

and since $-\Delta+I: W^{2, q^{\prime}+1}\left(\mathbb{R}^{N}\right) \rightarrow L^{q^{\prime}+1}\left(\mathbb{R}^{N}\right)$ is an isometric isomorphism (here $q^{\prime}>0$ is such that $\frac{1}{q+1}+\frac{1}{q^{\prime}+1}=1$ ), we have that $A:=\left\{-\Delta \varphi+\varphi: \varphi \in \mathcal{D}\left(\mathbb{R}^{N}\right)\right\}$ is a dense subspace in $L^{q^{\prime}+1}\left(\mathbb{R}^{N}\right)$, and our claim follows. Therefore, from (3.3)-(3.4),

$$
\int_{\mathbb{R}^{N}}|u|^{q+1} d x \leq \liminf _{n \rightarrow \infty} \int_{B_{n}}\left|u_{n}\right|^{q+1} d x \leq \int_{\mathbb{R}^{N}}|\bar{u}|^{q+1} d x .
$$

Lemma 3.5. The optimal constant $\alpha_{p, q}$ is attained; i.e., there exists $u \in E$ such that $|u|_{q+1}=1$ and $\|u\|^{\frac{p+1}{p}}=\alpha_{p, q}$.

Proof. Let $E_{r}=\{u \in E: u$ is radially symmetric $\}$ and set

$$
\alpha_{p, q, r}:=\inf \left\{\|u\|^{\frac{p+1}{p}}: u \in E_{r},|u|_{q+1}=1\right\} .
$$

From the compact embedding of $E_{r}$ into $L^{q+1}\left(\mathbb{R}^{N}\right)$, it follows that $\alpha_{p, q, r}$ is attained. Also, it is clear that

$$
\alpha_{p, q} \leq \alpha_{p, q, r}
$$

To prove the reverse inequality, take a minimizing sequence $\left(u_{n}\right) \subset E$ for $\alpha_{p, q}$. For each $n \in \mathbb{N}$, set $f_{n}:=L u_{n}$, let $\bar{u}_{n} \in E$ be the strong solution of $L \bar{u}_{n}=\left|f_{n}\right|^{*}$ in $\mathbb{R}^{N}$ and set $w_{n}:=\frac{\bar{u}_{n}}{\left|\bar{u}_{n}\right|_{q+1}}$. In particular, for each $n \in \mathbb{N}, w_{n} \in E_{r}$ and $\left|w_{n}\right|_{q+1}=1$. Moreover, by Lemma 3.4 .

$$
\alpha_{p, q, r} \leq\left\|w_{n}\right\|^{\frac{p+1}{p}}=\frac{\left\|\bar{u}_{n}\right\|^{\frac{p+1}{p}}}{\left|\bar{u}_{n}\right|_{q+1}^{\frac{p+1}{p}}} \leq \frac{\left\|\bar{u}_{n}\right\|^{\frac{p+1}{p}}}{\left|u_{n}\right|_{q+1}^{\frac{p+1}{p}}}=\left\|\bar{u}_{n}\right\|^{\frac{p+1}{p}}=\left\|u_{n}\right\|^{\frac{p+1}{p}} \rightarrow \alpha_{p, q}
$$

as $n \rightarrow \infty$. Hence the conclusion follows.

Proof of Theorems 1.8 1.9, The conclusion in Theorem 1.8 follows from what precedes. As for Theorem [1.9, we can argue as in the proof of Lemma 2.6. taking into account the regularity stated in Theorem 1.7. Here we rely on the auxiliary function $L w=|L u|$ in $\mathbb{R}^{N}$ and on the maximum principle as stated in 21, Theorem $3.5]$. 


\section{The problem in the Whole of $\mathbb{R}^{N}$ Revisited}

This section is devoted to a problem of the form

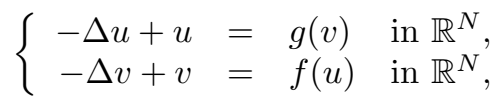

where $f, g \in C^{1}(\mathbb{R})$. Our structural assumptions on $f$ and $g$ are the following:

(fg1) $f(0)=g(0)=f^{\prime}(0)=g^{\prime}(0)=0$;

(fg2) there exist $p, q>1$ satisfying $(\mathrm{H} 1)$ such that

$$
|f(s)| \leq C\left(1+|s|^{q}\right) \text { and }|g(s)| \leq C\left(1+|s|^{p}\right)
$$

(fg3) there exists $\delta^{\prime}>0$ such that

$$
0<\left(1+\delta^{\prime}\right) f(s) s \leq f^{\prime}(s) s^{2} \text { and } 0<\left(1+\delta^{\prime}\right) g(s) s \leq g^{\prime}(s) s^{2} ;
$$

(fg4) $f$ and $g$ are odd symmetric functions;

(g1) there exist $a>0$ and $\alpha>0$ such that $a|s|^{\alpha+1} \leq g(s) s$ near the origin.

Of course, the assumption in (g1) could be replaced by a corresponding one on the function $f$. Observe also that, in order to be compatible, (fg2) and (fg3) imply $\delta^{\prime} \leq \min \{p-1, q-1\}$ whereas we can assume without loss of generality that $\delta^{\prime}<4 /(N-2)$ and $\alpha>p$.

These assumptions include, as a particular case, the system (1.4) treated in the previous section, provided $p, q>1$, and (H1) holds. More generally, we have in mind model non-linearities of the form $f(s) s=A|s|^{\alpha}+B|s|^{\beta}, g(s) s=A^{\prime}|s|^{\alpha^{\prime}}+B^{\prime}|s|^{\beta^{\prime}}$ with $A, B, A^{\prime}, B^{\prime}>0$ and $2<\beta \leq \alpha, 2<\beta^{\prime} \leq \alpha^{\prime}, 1 / \alpha+1 / \alpha^{\prime}>(N-2) / N$. As a further relevant example covered by our framework, we can allow $g(s) s$ (or $f(s) s)$ to behave near the origin like an arbitrary power $|s|^{r}(r>2$ arbitrarily large) provided it has "subcritical growth" at infinity, in the sense displayed in the condition (fg2). However, it is convenient for us to relax as much as possible the assumptions on $f$ and $g$, since our proofs will rely on truncation arguments, and we need to emphasize which properties of the non-linearities are preserved under these truncations.

We formally define the energy functional

$$
I(u, v)=\int_{\mathbb{R}^{N}}(\langle\nabla u, \nabla v\rangle+u v-F(u)-G(v)) d x,
$$

where $F(s):=\int_{0}^{s} f(\xi) d \xi$ and $G(s):=\int_{0}^{s} g(\xi) d \xi$. By a strong solution of the system we mean a pair $(u, v)$ satisfying (4.1) almost everywhere in $\mathbb{R}^{N}$, and $u \in$ $W^{2, \frac{p+1}{p}}\left(\mathbb{R}^{N}\right), v \in W^{2, \frac{q+1}{q}}\left(\mathbb{R}^{N}\right)$. Under assumptions (fg1)-(fg2), strong solutions of the system are in $L^{\infty}\left(\mathbb{R}^{N}\right) \cap H^{1}\left(\mathbb{R}^{N}\right) \cap C^{2}\left(\mathbb{R}^{N}\right)$ (see [33. Theorem 1(a)]), and therefore the following quantity (ground state critical level) is well defined:

$$
c_{0}\left(\mathbb{R}^{N}\right):=\inf \{I(u, v):(u, v) \text { is a non-zero strong solution of (4.1) }\} .
$$

It is known (see [30, Section 2]) that under assumptions (fg1)-(fg3) there exists a strong solution $(u, v)$ of the system, such that $u>0$ in $\mathbb{R}^{N}$ and $v>0$ in $\mathbb{R}^{N}$; it corresponds to a ground state critical level for the system $-\Delta u+u=g\left(v^{+}\right),-\Delta v+$ $v=f\left(u^{+}\right)$, where $s^{+}:=\max \{s, 0\} \forall s \in \mathbb{R}$. The following theorem complements this existence result; it implies that, under the conditions below, the energy level of the solution $(u, v)$ found in $\left[30\right.$ is precisely $c_{0}\left(\mathbb{R}^{N}\right)$. 
Theorem 4.1. Assume (fg1)-(fg4) and $(\mathrm{g} 1)$. Then $c_{0}\left(\mathbb{R}^{N}\right)$ is attained at some strong solution $(u, v)$ of (4.1). Moreover, any such ground state solution $(u, v)$ is such that uv $>0$ in $\mathbb{R}^{N}$.

In order to make more apparent the relation between Theorem 4.1 and Theorems 1.8 1.9. let us consider for a moment the special case where $f(s) s=|s|^{q+1}$ and $g(s) s=|s|^{p+1}$ with $p q>1$ and $(\mathrm{H} 1)$ and let $c_{J}$ be the ground state level as defined in the previous section. Then we have that $c_{J}=c_{0}\left(\mathbb{R}^{N}\right)$. This follows from Theorem 1.7 and Proposition 3.1. Theorem 4.1 can thus be seen as a partial extension of Theorems 1.8 [1.9 to problems with non-pure power non-linearities; the results are not completely comparable, since Theorem 4.1 does not cover the case where $p q>1$. We also mention that a statement slightly more general than Proposition 3.2 can be derived, in case $p, q>1$ and (H1) are satisfied, provided we replace the space $H^{1}\left(\mathbb{R}^{N}\right) \times H^{1}\left(\mathbb{R}^{N}\right)$ with suitable Sobolev fractional spaces $E^{s} \times E^{t}$ as in the approach followed in [16, 22].

As a further remark, suppose that (fg2) and (fg3) hold with $p(N-2)<(N+2)$, $q(N-2)<(N+2), p, q>1$. Then the energy functional is well defined in $H^{1}\left(\mathbb{R}^{N}\right) \times H^{1}\left(\mathbb{R}^{N}\right)$, and by the usual elliptic regularity theory we see that

$$
c_{0}\left(\mathbb{R}^{N}\right)=\inf \left\{I(u, v): u, v \in H^{1}\left(\mathbb{R}^{N}\right),(u, v) \neq 0, I^{\prime}(u, v)=0\right\} .
$$

In particular, as shown in [30, in this case the existence of a ground state is insured. Moreover, see [30, Proposition 2.4], we have the following variational characterizations of $c_{0}\left(\mathbb{R}^{N}\right)$ :

$$
\begin{aligned}
c_{0}\left(\mathbb{R}^{N}\right)= & \inf \left\{I(u, v): u, v \in H^{1}\left(\mathbb{R}^{N}\right),(u, v) \neq(0,0),\right. \\
& \left.I^{\prime}(u, v)(u+\psi, v-\psi)=0 \quad \forall \psi \in H^{1}\left(\mathbb{R}^{N}\right)\right\} \\
= & \inf _{(u, v) \neq(0,0)} \sup \left\{I(t u+\psi, t v-\psi): t \geq 0, \psi \in H^{1}\left(\mathbb{R}^{N}\right)\right\} \\
= & \inf _{u \neq-v} \sup \left\{I(t u+\psi, t v-\psi): t \geq 0, \psi \in H^{1}\left(\mathbb{R}^{N}\right)\right\} .
\end{aligned}
$$

In proving Theorem 4.1 we will exploit both the variational characterizations of $c_{J}$ and $c_{0}\left(\mathbb{R}^{N}\right)$. However, under our general assumptions (fg1)-(fg4) and (g1), $c_{J}$ is not properly defined and the above characterization of $c_{0}\left(\mathbb{R}^{N}\right)$ is meaningless. To overcome this, we will prove an apparently weaker result.

Proposition 4.2. Assume (fg1)-(fg4) and (g1) hold. Suppose moreover that

$($ fg2' $)$ there exist $1<r<(N+2) /(N-2), 0<\ell_{1}<\infty$ and $0<\ell_{2}<\infty$ such that

$$
\lim _{|s| \rightarrow \infty} f^{\prime}(s) /|s|^{r-1}=\ell_{1} \text { and } \lim _{|s| \rightarrow \infty} g^{\prime}(s) /|s|^{r-1}=\ell_{2},
$$

and $C>0$ such that for every $s \in \mathbb{R}$,

$$
0 \leq f(s) s \leq C|s|^{r+1} \text { and } 0 \leq g(s) s \leq C|s|^{r+1} .
$$

Then the conclusion of Theorem 4.1 holds.

We anticipate that the additional assumption $\left(\mathrm{fg} 2^{\prime}\right)$ will allow us to play with the above characterization of $c_{0}\left(\mathbb{R}^{N}\right)$. Before turning to the proof of Proposition 4.2. we first show how the conclusion of Theorem 4.1 can be deduced from it. To this aim we introduce a family of truncated problems.

Assumption (fg3) implies that for every $s \geq 1, f(s) \geq f(1) s^{1+\delta^{\prime}}$ whereas $f(s) \leq$ $f(1) s^{1+\delta^{\prime}}$ for every $s \leq 1$. On the other hand, assumption (fg2) yields the existence 
of a sequence $R_{n} \rightarrow \infty$ such that $f^{\prime}\left(R_{n}\right) / R_{n}^{q-1}$ is bounded. Accordingly, let $f_{n}$ be the odd symmetric $C^{1}$ function defined by

$$
f_{n}(s)=\left\{\begin{array}{cl}
f(s) & \text { if } 0 \leq s \leq R_{n}, \\
\frac{f^{\prime}\left(R_{n}\right)}{\left(1+\delta^{\prime}\right) R_{n}^{\delta^{\prime}}}\left(s^{1+\delta^{\prime}}-R_{n}^{1+\delta^{\prime}}\right)+f\left(R_{n}\right) & \text { if } s \geq R_{n},
\end{array}\right.
$$

where $\delta^{\prime}$ is given by (fg3). Define $g_{n}$ in a similar way, namely

$$
g_{n}(s)=\left\{\begin{array}{cl}
g(s) & \text { if } 0 \leq s \leq R_{n}^{\prime}, \\
\frac{g^{\prime}\left(R_{n}^{\prime}\right)}{\left(1+\delta^{\prime}\right) R_{n}^{\prime \delta^{\prime}}}\left(s^{1+\delta^{\prime}}-R_{n}^{\prime 1+\delta^{\prime}}\right)+g\left(R_{n}^{\prime}\right) & \text { if } s \geq R_{n}^{\prime},
\end{array}\right.
$$

where the sequence $R_{n}^{\prime} \rightarrow \infty$ is such that $g^{\prime}\left(R_{n}\right) / R_{n}^{p-1}$ is bounded.

Then, by construction, there exists a universal constant $C>0$ such that for every $|s| \geq 1$,

$$
\left|f_{n}(s)\right| \leq C|s|^{q} \text { and }\left|g_{n}(s)\right| \leq C|s|^{p} .
$$

Simple computations also show that the properties displayed in (fg1) $-(\mathrm{fg} 4)$ and (g1) are preserved under these truncations. At last, observe that there exists $C_{n}>0$ such that for every $s$,

$$
0 \leq f_{n}(s) s \leq C_{n}|s|^{2+\delta^{\prime}} \text { and } 0 \leq g_{n}(s) s \leq C_{n}|s|^{2+\delta^{\prime}} .
$$

Consider now the auxiliary systems

$$
\left\{\begin{aligned}
-\Delta u+u & =g_{n}(v) \quad \text { in } \mathbb{R}^{N} \\
-\Delta v+v & =f_{n}(u) \text { in } \mathbb{R}^{N}
\end{aligned}\right.
$$

Since the growth of $f_{n}$ and $g_{n}$ is subcritical (in the sense of the equation), as pointed out above, we can define the associated energy functional in $H^{1}\left(\mathbb{R}^{N}\right) \times H^{1}\left(\mathbb{R}^{N}\right)$. Now, as explained in [30, Section 2], working with these truncated problems $\left(S_{n}\right)$ gives rise to ground state critical levels $c_{0}^{n}\left(\mathbb{R}^{N}\right)$. However, these critical levels are bounded uniformly with respect to $n$, and so the corresponding ground state solutions are bounded in $L^{\infty}\left(\mathbb{R}^{N}\right)$ uniformly in $n$ and are therefore (strong) solutions of our original system. We make this precise in the next lemma.

Lemma 4.3. The set of ground state solutions of the systems $S_{n}$ is bounded in the $L^{\infty} \times L^{\infty}$-topology.

Proof. As explained in [30, Lemma 5.1], since the critical levels associated to the auxiliary systems $\left(S_{n}\right)$ are, by construction, bounded uniformly with respect to $n$, we infer that there exists $C>0$ such that

$$
\int_{\mathbb{R}^{N}} f_{n}\left(u_{n}\right) u_{n}+\int_{\mathbb{R}^{N}} g_{n}\left(v_{n}\right) v_{n} \leq C
$$

for any ground state solution $\left(u_{n}, v_{n}\right) \in H^{1}\left(\mathbb{R}^{N}\right) \times H^{1}\left(\mathbb{R}^{N}\right)$. Since $f_{n}$ and $g_{n}$ satisfy (4.5) for every $s$, we infer that $u_{n}, v_{n} \in W^{2,\left(\delta^{\prime}+2\right) /\left(\delta^{\prime}+1\right)}\left(\mathbb{R}^{N}\right)$ for each $n$; i.e., these are strong solutions of the truncated system. Observe now that since the truncated non-linearities satisfy the assumptions of Proposition 4.2, any ground state is positive. Then, by [17, Theorem 2.2], both $u_{n}$ and $v_{n}$ decay exponentially; 
in particular, $u_{n}, v_{n} \in L^{1}\left(\mathbb{R}^{N}\right)$. Integrating the first equation of the system, we find that, for a small $\delta>0$,

$$
\begin{aligned}
\left\|u_{n}\right\|_{L^{1}\left(\mathbb{R}^{N}\right)} & =\int_{\mathbb{R}^{N}} u_{n}=\int_{\mathbb{R}^{N}} g_{n}\left(v_{n}\right) \leq \frac{1}{4} \int_{v_{n} \leq \delta} v_{n}+\int_{v_{n} \geq \delta} g_{n}\left(v_{n}\right) \\
& \leq \frac{1}{4} \int_{\mathbb{R}^{N}} v_{n}+\frac{1}{\delta} \int_{\mathbb{R}^{N}} g_{n}\left(v_{n}\right) v_{n} \leq \frac{1}{4} \int_{\mathbb{R}^{N}} v_{n}+C^{\prime}
\end{aligned}
$$

Arguing in the same way with the second equation of the system, we deduce that

$$
\left\|u_{n}\right\|_{L^{1}\left(\mathbb{R}^{N}\right)}+\left\|v_{n}\right\|_{L^{1}\left(\mathbb{R}^{N}\right)} \leq C
$$

with $C$ independent of $n$. Next, by observing that $g_{n}\left(v_{n}\right)^{(p+1) / p} \leq C g_{n}\left(v_{n}\right) v_{n}$ on

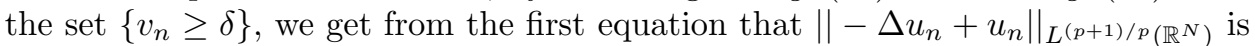
bounded. Also using the second equation, we conclude that $\left(u_{n}, v_{n}\right)$ is bounded in $W^{2, \frac{p+1}{p}}\left(\mathbb{R}^{N}\right) \times W^{2, \frac{q+1}{q}}\left(\mathbb{R}^{N}\right)$. At last, by then using a bootstrap argument (see 33 , pp. 1450-1451]), we conclude that $\left(u_{n}, v_{n}\right)$ is bounded in $L^{\infty}\left(\mathbb{R}^{N}\right) \times L^{\infty}\left(\mathbb{R}^{N}\right)$, as claimed.

It follows from the preceding lemma that the original system and the truncated ones share the same ground state critical levels, provided $n$ is taken sufficiently large. We can therefore complete the proof of Theorem 4.1.

Proof of Theorem 4.1. We first recall that, as emphasized above, strong solutions of the system (4.1) are in $L^{\infty}\left(\mathbb{R}^{N}\right)$. Let $R>0$ be the bound obtained in Lemma 4.3 and choose $n$ large enough so that $R_{n}>R$. Then, arguing by contradiction, it is clear that if $(u, v)$ is a ground state solution of $\left(S_{n}\right)$, then $(u, v)$ is a ground state solution of (4.1) and reciprocally. Hence the conclusion follows from Proposition 4.2 .

Remark 4.4. In section [5] we deal with a problem similar to (4.1) in an exterior domain $\Omega$, under the assumptions (fg1)-(fg6), where

(fg5) both $f^{\prime}(s)$ and $g^{\prime}(s)$ are non-decreasing functions for $s>0$;

(fg6) there exists $C_{0}>0$ such that for all $s \in \mathbb{R}$,

$$
f^{\prime}(2 s) s^{2} \leq C_{0} f(s) s \text { and } g^{\prime}(2 s) s^{2} \leq C_{0} g(s) s .
$$

Observe that (fg6) implies (g1). The conclusion in Lemma 4.3, which leads to the fact that in proving (extended versions of) Theorems 1.10 and 1.11 we may assume that (fg $2^{\prime}$ ) holds, remains true in this case. This can be checked by going through the above argument and by also taking into account the following three points:

(i) the constant $C_{0}$ which appears in (fg6) is independent of $R_{n}$ and $R_{n}^{\prime}$;

(ii) in case $u_{n}>0, \int_{\Omega}\left(-\Delta u_{n}\right) \geq 0$ by the Hopf lemma;

(iii) the argument in the proof of Lemma 4.3 concerning the bound of $\left(u_{n}, v_{n}\right)$ in the space $W^{2, \frac{p+1}{p}}\left(\mathbb{R}^{N}\right) \times W^{2, \frac{q+1}{q}}\left(\mathbb{R}^{N}\right)$ does not apply for sign changing solutions, but it does apply in case for every $n \in \mathbb{N}$ and $x \in \Omega$,

$$
g_{n}\left(v_{n}(x)\right)^{(p+1) / p} \leq C g_{n}\left(v_{n}(x)\right) v_{n}(x)
$$

and a similar inequality holds for $f_{n}$. This is the case considered in Theorem 1.11 
We also mention that in the present section we have avoided arguments based on the radial symmetry of the solutions or Palais-Smale sequences, as we did in section 3 , so that our conclusions can be straightforwardly adapted to the case of an exterior domain.

Our main concern in the remainder of this section is the proof of Proposition 4.2. We will proceed in several steps. Unless otherwise stated, we assume from now on that (fg4)-(g1) holds, as well as $\left(\mathrm{fg} 2^{\prime}\right)$. In particular, the energy functional

$$
I(u, v)=\int_{\mathbb{R}^{N}}(\langle\nabla u, \nabla v\rangle+u v-F(u)-G(v))
$$

is now a well-defined $C^{2}$ functional in $H^{1}\left(\mathbb{R}^{N}\right) \times H^{1}\left(\mathbb{R}^{N}\right)$. In the sequel we will also work in large balls $B_{R}(0) \subset \mathbb{R}^{N}$; that is, we will consider the problem

$$
-\Delta u+u=g(v), \quad-\Delta v+v=f(u), \quad u, v \in H_{0}^{1}\left(B_{R}(0)\right) .
$$

We denote by $c_{0}\left(B_{R}(0)\right)$ the corresponding ground state critical level associated to the energy functional

$$
I_{R}(u, v):=\int_{B_{R}(0)}(\langle\nabla u, \nabla v\rangle+u v-F(u)-G(v)), \quad u, v \in H_{0}^{1}\left(B_{R}(0)\right),
$$

namely

$$
c_{0}\left(B_{R}(0)\right):=\inf \left\{I(u, v): u, v \in H_{0}^{1}\left(B_{R}(0)\right),(u, v) \neq(0,0), I^{\prime}(u, v)=0\right\} .
$$

We stress that since the Palais-Smale condition holds, this is a well-defined positive critical level for the functional $I_{R}$ (see e.g. [30, Section 2]). Let $h(s):=g^{-1}(s)$ and $H(s):=\int_{0}^{s} h(\xi) d \xi$. We recall that, by assumption, for every $s \in \mathbb{R}$,

$$
0 \leq g(s) s \leq C|s|^{r+1},
$$

while

$$
g(s) s \geq c|s|^{r+1} \text { for }|s| \geq 1 \text { and } g(s) s \geq a|s|^{\alpha+1} \text { for }|s| \leq 1,
$$

where $\alpha>0$ is a constant that we can assume to be large, namely $\alpha>r$. As a consequence, we deduce that there exist $a_{0}, C>0$ such that for every $s \in \mathbb{R}$,

$$
a_{0}|s|^{(r+1) / r} \leq h(s) s \leq C\left(|s|^{(r+1) / r}+|s|^{(\alpha+1) / \alpha}\right) .
$$

Accordingly, define the Banach space $E:=W^{2, \frac{r+1}{r}}\left(\mathbb{R}^{N}\right) \cap W^{2, \frac{\alpha+1}{\alpha}}\left(\mathbb{R}^{N}\right)$, equipped with the norm $\| u||:=|L u|_{(r+1) / r}+|L u|_{(\alpha+1) / \alpha}$, where $L u:=-\Delta u+u$ and $|u|_{s}:=$ $\left(\int_{\mathbb{R}^{N}}|u|^{s}\right)^{1 / s}$. We observe that $E$ is continuously imbedded in $L^{2}\left(\mathbb{R}^{N}\right) \cap L^{r+1}\left(\mathbb{R}^{N}\right)$. Let $J: E \rightarrow \mathbb{R}$ be given by

$$
J(u):=\int_{\mathbb{R}^{N}} H(L u)-\int_{\mathbb{R}^{N}} F(u), \quad u \in E .
$$

Then $J$ is a $C^{1}$ functional and

$$
J^{\prime}(u) \varphi=\int_{\mathbb{R}^{N}} h(L u) L \varphi-\int_{\mathbb{R}^{N}} f(u) \varphi, \quad \forall u, \varphi \in E .
$$

Our main goal is to prove Lemma 4.12 below by using the least energy critical level of $J$. However, under our assumptions it is not clear whether such least energy critical level is well defined. Due to this fact, we first restrict ourselves to the bounded domain case (cf. Lemma 4.8) and then carefully pass to the limit in increasing large balls of $\mathbb{R}^{N}$. Two basic properties of the functional $J$ are presented in our next lemma. 
Lemma 4.5. (a) For every $u \in E, u \neq 0, J(t u) \rightarrow-\infty$ as $t \rightarrow+\infty$.

(b) If $J^{\prime}(u) u=0$, then $J(u)=\sup _{t \geq 0} J(t u)$.

Proof. Property $(a)$ easily follows from the observation that $F(s) \geq c_{1}|s|^{r+1}-c_{2} s^{2}$ for some $c_{1}, c_{2}>0$ (cf. $\left.\left(\mathrm{fg}^{\prime}\right)\right)$ and $\alpha>1 / r$.

As for $(b)$, let us fix $u \in E \backslash\{0\}$ such that $J^{\prime}(u) u=0$. We now use assumption (fg3) in a weaker form. Namely, it follows from (fg3) that there exists $\mu:=-\delta^{\prime} /(1+$ $\left.\delta^{\prime}\right) \in\left(-1, \delta^{\prime}\right)$ such that

$$
0<\left(1+\delta^{\prime}\right) f(s) s \leq f^{\prime}(s) s^{2} \text { and } 0<\frac{1}{1+\mu} g(s) s \leq g^{\prime}(s) s^{2} .
$$

Using the very definition of $h$ and the last inequality, we infer that for every $s \neq 0$,

$$
0<h^{\prime}(s) s^{2} \leq(1+\mu) h(s) s .
$$

We rephrase this as follows. Let $h_{0}(s):=h(s) s$ and $f_{0}(s):=f(s) s$. Then $f_{0}, h_{0} \in$ $C^{1}(\mathbb{R})$ and for every $s \in \mathbb{R}$,

$$
0 \leq h_{0}^{\prime}(s) s \leq(2+\mu) h_{0}(s) \quad \text { and } \quad f_{0}^{\prime}(s) s \geq\left(2+\delta^{\prime}\right) f_{0}(s) .
$$

Let $\theta(t):=J(t u), t \geq 0$. By assumption, $\theta^{\prime}(1)=0$. In order to prove that $J(u)=\sup _{t>0} J(t u)$ it is enough to show that if $\theta^{\prime}\left(t_{0}\right)=0$ and $t_{0}>0$, then $\left(t_{0}-t\right) \theta^{\prime}(t)>0$ for every $t \neq t_{0}$ close to $t_{0}$. For that purpose, we define $\gamma(t):=t \theta^{\prime}(t)$ for $t \geq 0$. Namely,

$$
\gamma(t)=\int_{\mathbb{R}^{N}} h_{0}(t L u)-\int_{\mathbb{R}^{N}} f_{0}(t u)
$$

We observe that there exists $C>0$ such that for every $s \in \mathbb{R}$,

$$
0 \leq h_{0}(s) \leq C\left(|s|^{(r+1) / r}+|s|^{(\alpha+1) / \alpha}\right)
$$

and therefore, by (4.8), we infer that there exists $C^{\prime}>0$ such that for every $s \in \mathbb{R}$,

$$
0 \leq h_{0}^{\prime}(s) s \leq C^{\prime}\left(|s|^{(r+1) / r}+|s|^{(\alpha+1) / \alpha}\right) .
$$

In particular, $\gamma$ is of class $C^{1}$ and

$$
t \gamma^{\prime}(t)=\int_{\mathbb{R}^{N}} h_{0}^{\prime}(t L u) t L u-\int_{\mathbb{R}^{N}} f_{0}^{\prime}(t u) t u .
$$

Now, using the inequalities (4.8), we conclude that

$$
t \gamma^{\prime}(t) \leq(2+\mu) \gamma(t)+\left(\mu-\delta^{\prime}\right) \int_{\mathbb{R}^{N}} f_{0}(t u)
$$

where we recall that $\mu<\delta^{\prime}$. By assumption, $\gamma\left(t_{0}\right)=0$, and therefore $\gamma^{\prime}\left(t_{0}\right)<0$. This implies $\left(t_{0}-t\right) \gamma(t)>0$ for every $t \neq t_{0}$ close to $t_{0}$, yielding our claim.

Proposition 4.6. Assume (fg1), (fg2'), (fg3), (fg4), (g1). The following statements are equivalent:

(i) $(u, v)$ is a critical point of $I$.

(ii) $u$ is a critical point of $J$ and $v=h(L u)$.

(iii) $u, v \in E$ and $(u, v)$ is a strong solution of (4.1).

(iv) $u, v$ are as in (iii) and $u, v \in W^{2, s}\left(\mathbb{R}^{N}\right)$ for all $1<s<\infty$.

In any such case, $I(u, v)=J(u)$. 
The proof for Proposition 4.6 is similar to the proof of Theorem 1.7. The identity $I(u, v)=J(u)$ follows as in (4.9) below.

We now turn our attention to a similar framework, but restricted to large balls $B_{R}(0) \subset \mathbb{R}^{N}$. In this case, since $\alpha \geq r$, we can replace the space $E$ by the Sobolev space $E_{R}:=W^{2, \frac{r+1}{r}}\left(B_{R}(0)\right) \cap W_{0}^{1, \frac{r+1}{r}}\left(B_{R}(0)\right)$ and work with the $C^{1}$ functional

$$
J_{R}(u):=\int_{B_{R}(0)} H(L u)-\int_{B_{R}(0)} F(u), \quad u \in E_{R} .
$$

Proposition 4.7. Assume (fg1), (fg2'), (fg3), (fg4), (g1). The following statements are equivalent:

(i) $(u, v)$ is a critical point of $I_{R}$.

(ii) $u$ is a critical point of $J_{R}$ and $v=h(L u)$.

(iii) $u, v \in E_{R}$ and $(u, v)$ is a strong solution of (4.6).

(iv) $u, v$ are as in (iii) and $u, v \in W^{2, s}\left(B_{R}(0)\right)$ for all $1 \leq s<\infty$.

(v) $u, v \in C^{2}\left(\bar{B}_{R}(0)\right) \cap C_{0}\left(\bar{B}_{R}(0)\right)$ and $(u, v)$ is a classical solution of (4.6).

(vi) $u, v$ are as in (v) and $u, v \in C^{2, \alpha}\left(\bar{B}_{R}(0)\right)$ for all $0<\alpha \leq 1$.

In any such case, $I_{R}(u, v)=J_{R}(u)$.

The proof for Proposition 4.7 is similar to the proof of Theorem 1.7 and Proposition 4.6 .

Lemma 4.8. For every $R>0$,

$$
c_{0}\left(B_{R}(0)\right)=\inf \left\{J_{R}(u): u \in E_{R} \backslash\{0\}, J_{R}^{\prime}(u) u=0\right\} .
$$

Proof. We define $\mathcal{N}:=\left\{u \in E_{R} \backslash\{0\}, J_{R}^{\prime}(u) u=0\right\}$ and $c_{1}\left(B_{R}(0)\right):=\inf _{\mathcal{N}} J_{R}$. Let $(u, v) \in H_{0}^{1}\left(B_{R}(0)\right) \times H_{0}^{1}\left(B_{R}(0)\right)$ be a solution of the system associated to the critical level $c_{0}\left(B_{R}(0)\right)$. By Proposition 4.7, $u \in E_{R}, v=h(L u)$, and $J_{R}^{\prime}(u)=0$. This shows that $c_{0}\left(B_{R}(0)\right) \geq c_{1}\left(B_{R}(0)\right)$.

In order to prove the reversed inequality, it is sufficient to show that $c_{1}\left(B_{R}(0)\right)$ is a critical value of the functional $J_{R}$; that is, there exists $u \in E_{R}$ such that $J_{R}(u)=c_{1}\left(B_{R}(0)\right)$ and $J_{R}^{\prime}(u)=0$. Then, by Proposition 4.7. $u \in H_{0}^{1}\left(B_{R}(0)\right)$, $v:=h(L u) \in H_{0}^{1}\left(B_{R}(0)\right)$, and the pair $(u, v)$ is a non-zero solution of the system. Clearly, in this case, observing that for every $s \in \mathbb{R}$ we have $G(h(s))=h(s) s-H(s)$, we infer that

$$
\begin{aligned}
c_{0}\left(B_{R}(0)\right) \leq I_{R}(u, v) & =\int_{B_{R}(0)}(\langle\nabla u, \nabla v\rangle+u v-F(u)-G(v)) \\
= & \int_{B_{R}(0)}(\langle\nabla u, \nabla v\rangle+u v-F(u)+H(L u)-g(v) v) \\
& =\int_{B_{R}(0)}(-F(u)+H(L u)):=J_{R}(u)=c_{1}\left(B_{R}(0)\right),
\end{aligned}
$$

and the lemma follows.

In order to prove that $c_{1}\left(B_{R}(0)\right)$ is a critical value of $J_{R}$, one can first argue as in the proof of Lemma4.5 to prove that any $u \in \mathcal{N}$ does satisfy $J_{R}(u)=\sup _{t \geq 0} J_{R}(t u)$ and for every $u \in E_{R}$,

$$
(2+\mu) J_{R}(u) \geq J_{R}^{\prime}(u) u+\left(\delta^{\prime}-\mu\right) \int_{B_{R}(0)} F(u),
$$

for some $\mu<\delta^{\prime}$. Moreover, we can write $\mathcal{N}=\left\{u \in E_{R}, u \neq 0: T(u)=0\right\}$, where $T$ is the $C^{1}$ map defined by $T(u):=\int_{B_{R}(0)} h(L u) L u-\int_{B_{R}(0)} f(u) u$, and for every 
$u \in \mathcal{N}, T^{\prime}(u) u<0$. In particular, the manifold $\mathcal{N}$ is a natural constraint for the functional $J_{R}$. Finally, since there exists $a_{0}>0$ such that $a_{0}|s|^{(p+1) / p} \leq h(s) s$, we deduce that if $\rho>0$ is sufficiently small, then

$$
\inf \left\{J_{R}(u): u \in E_{R},\|u\|=\rho\right\}>0 .
$$

Recall that, here, we use the norm $\|u\|:=|L u|_{(r+1) / r}$ rather than $|L u|_{(r+1) / r}+$ $|L u|_{(\alpha+1) / \alpha}$. In particular, $c_{1}\left(B_{R}(0)\right)>0$. Now, let $\left(u_{n}\right)_{n} \subset \mathcal{N}$ be a minimizing sequence for $c_{1}\left(B_{R}(0)\right)$. The inequality (4.10) shows that we have the estimate

$$
\int_{B_{R}(0)} F\left(u_{n}\right) \leq C\left(1+\left\|u_{n}\right\|\right),
$$

and therefore

$$
\left\|u_{n}\right\|^{(r+1) / r} \leq C^{\prime} \int_{B_{R}(0)} H\left(L u_{n}\right) \leq C^{\prime \prime}\left(1+\left\|u_{n}\right\|\right) .
$$

So $\left(u_{n}\right)_{n}$ is bounded and, up to a subsequence, we have that $u_{n} \rightarrow u$ weakly in $E_{R}$. Since $\int_{B_{R}(0)} h\left(L u_{n}\right) L u_{n}=\int_{B_{R}(0)} f\left(u_{n}\right) u_{n}$, it follows from the fact that $c_{1}\left(B_{R}(0)\right)>0$ and the compact embedding of $E_{R}$ into $L^{r+1}\left(B_{R}(0)\right)$ that $u \neq 0$. At last, let us fix $\bar{t}>0$ such that $\bar{t} u \in \mathcal{N}$. Since $H$ is a convex function and since $\int_{B_{R}(0)} F\left(\bar{t} u_{n}\right) \rightarrow \int_{B_{R}(0)} F(\bar{t} u)$, we have that $J_{R}(\bar{t} u) \leq \lim \inf J_{R}\left(\bar{t} u_{n}\right)$. By recalling that $\left(u_{n}\right)_{n} \subset \mathcal{N}$, we deduce that

$$
c_{1}\left(B_{R}(0)\right) \leq J_{R}(\bar{t} u) \leq \liminf J_{R}\left(\bar{t} u_{n}\right) \leq \liminf J_{R}\left(u_{n}\right)=c_{1}\left(B_{R}(0)\right) .
$$

This shows that $w:=\bar{t} u \in \mathcal{N}$ does satisfy $J_{R}(w)=c_{1}\left(B_{R}(0)\right)$. Since $\mathcal{N}$ is a natural constraint for the functional $J_{R}$, we conclude that $J_{R}^{\prime}(w)=0$, and this completes the proof.

Lemma 4.9. For any ground state solution $(u, v) \in H_{0}^{1}\left(B_{R}(0)\right) \times H_{0}^{1}\left(B_{R}(0)\right)$ of the system, we have that $u v>0$ in $B_{R}(0)$.

Proof. By Proposition 4.7 we have that $u \in E_{R}$, and it follows from Lemma 4.8 that

$$
J_{R}(u)=\min \left\{J_{R}(w): w \in E_{R} \backslash\{0\}, J_{R}^{\prime}(w) w=0\right\} .
$$

Let $w:=L^{-1}(|L u|) \in E_{R}$. It follows from the maximum principle that $w>0$ and $w \geq|u|$. Let us fix $t>0$ such that $J_{R}^{\prime}(t w)(t w)=0$. Since $F$ and $H$ are even functions and thanks to an analogous version of Lemma 4.5 (b) for $J_{R}$, we deduce that

$$
J_{R}(u) \leq J_{R}(t w)=J_{R}(t u)+\int_{B_{R}(0)}(F(t|u|)-F(t w)) \leq J_{R}(t u) \leq J_{R}(u) .
$$

Thus $\int_{B_{R}(0)}(F(t|u|)-F(t w))=0$. Since $w \geq|u|$, this implies $|u|=w>0$. We conclude that $u$ does not change sign. Going back to the system, we see that the same holds for $v$; in fact, by the maximum principle, $u$ and $v$ have the same sign.

Lemma 4.10. We have that $c_{0}\left(B_{R}(0)\right) \geq c_{0}\left(B_{\bar{R}}(0)\right)$ for every $\bar{R}>R>0$.

Proof. This is essentially proved in [28]. Indeed, let $(u, v) \in H_{0}^{1}\left(B_{R}(0)\right) \times H_{0}^{1}\left(B_{R}(0)\right)$ be a ground state solution with energy level $c_{0}\left(B_{R}(0)\right)$. By Lemma 4.9] we can choose $(u, v)$ in such a way that $u>0$ and $v>0$ in $B_{R}(0)$. We denote by $\bar{u}, \bar{v}$ their extensions by zero, so that $(\bar{u}, \bar{v}) \in H_{0}^{1}\left(B_{\bar{R}}(0)\right) \times H_{0}^{1}\left(B_{\bar{R}}(0)\right)$. Since $u>0$ and $v>0$ in $B_{R}(0)$, it follows as in [28, Proposition 2.2] that $I_{\bar{R}}(\bar{u}, \bar{v}) \geq c_{0}\left(B_{\bar{R}}(0)\right)$. Since $I_{\bar{R}}(\bar{u}, \bar{v})=I(u, v)$, we are done. 
Remark 4.11. An alternate, more direct, proof of Lemma 4.10 goes as follows. As shown in the proof of Lemma 4.8, it is enough to prove that $c_{1}\left(B_{R}(0)\right) \geq c_{1}\left(B_{\bar{R}}(0)\right)$. Let $u \in E_{R}, u>0$, be such that $J_{R}(u)=c_{1}\left(B_{R}(0)\right)$, and denote by $\overline{L u}$ the extension by zero of $L u$. By letting $w \in E_{\bar{R}}$ be such that $L w=\overline{L u}$ in $B_{\bar{R}}(0)$ and $w=0$ on $\partial B_{\bar{R}}(0)$ it follows that $w \geq u$, and so we have for every $t \geq 0$,

$$
J_{\bar{R}}(t w) \leq J_{R}(t u) \leq J_{R}(u)=c_{1}\left(B_{R}(0)\right),
$$

yielding that $c_{1}\left(B_{\bar{R}}(0)\right) \leq c_{1}\left(B_{R}(0)\right)$.

Let $c_{0}\left(\mathbb{R}^{N}\right)$ be as in (4.2). Since (fg2 $\left.2^{\prime}\right)$ holds, the equalities in (4.3) and (4.4) hold.

Lemma 4.12. We have that $c_{0}\left(B_{R}(0)\right) \geq c_{0}\left(\mathbb{R}^{N}\right)$ for every $R>0$.

Proof. We introduce the auxiliary problem

$$
-\Delta u+u=g(v), \quad-\Delta v+v=\frac{1}{1+\lambda|x|^{2}} f(u), \quad u, v \in H_{0}^{1}\left(B_{R}(0)\right),
$$

where $\lambda>0$ is a small parameter, and denote by $c_{0}^{\lambda}\left(B_{R}(0)\right)$ the associated ground state critical level. We can repeat word by word the arguments in the proofs of Lemmas 4.8, 4.9 and 4.10, to conclude that $c_{0}^{\lambda}\left(B_{R}(0)\right) \geq c_{0}^{\lambda}\left(B_{\bar{R}}(0)\right)$ for every $\bar{R}>R>0$. We consider a similar auxiliary problem in the whole space $\mathbb{R}^{N}$, we denote by $I_{\lambda}$ the associated energy functional,

$$
I_{\lambda}(u, v)=\int_{\mathbb{R}^{N}}\left(\langle\nabla u, \nabla v\rangle+u v-\frac{1}{1+\lambda|x|^{2}} F(u)-G(v)\right), \quad u, v \in H^{1}\left(\mathbb{R}^{N}\right),
$$

and we let

$$
\begin{aligned}
c_{0}^{\lambda}\left(\mathbb{R}^{N}\right):= & \inf \left\{I_{\lambda}(u, v): u, v \in H^{1}\left(\mathbb{R}^{N}\right),(u, v) \neq(0,0),\right. \\
& \left.I_{\lambda}^{\prime}(u, v)(u, v)=0, I_{\lambda}^{\prime}(u, v)(\psi,-\psi)=0 \forall \psi \in H^{1}\left(\mathbb{R}^{N}\right)\right\} \\
= & \inf _{(u, v) \neq(0,0)} \sup \left\{I_{\lambda}(t u+\psi, t v-\psi): t \geq 0, \psi \in H^{1}\left(\mathbb{R}^{N}\right)\right\} .
\end{aligned}
$$

Since $I \leq I_{\lambda}$ we deduce from (4.4) and (4.11) that $c_{0}\left(\mathbb{R}^{N}\right) \leq c_{0}^{\lambda}\left(\mathbb{R}^{N}\right)$. Similarly, $c_{0}\left(B_{R}(0)\right) \leq c_{0}^{\lambda}\left(B_{R}(0)\right)$. Moreover, using the compactness of the embedding of $H_{0}^{1}\left(B_{R}(0)\right)$ into $L^{r+1}\left(B_{R}(0)\right)$ it is easily shown that $c_{0}^{\lambda}\left(B_{R}(0)\right) \rightarrow c_{0}\left(B_{R}(0)\right)$ as $\lambda \rightarrow 0$. Therefore the conclusion follows if we show that $c_{0}^{\lambda}\left(B_{R}(0)\right) \geq c_{0}^{\lambda}\left(\mathbb{R}^{N}\right)$ for every $\lambda, R>0$.

In order to prove this, let us fix $\lambda>0$, any sequence $R_{n} \rightarrow \infty$ and critical points $\left(u_{n}, v_{n}\right) \neq(0,0)$ associated to the critical levels $c_{0}^{\lambda}\left(B_{R_{n}}(0)\right)$. As pointed out above, we have that $c_{0}^{\lambda}\left(B_{R}(0)\right) \geq c_{0}^{\lambda}\left(B_{R_{n}}(0)\right)$ along the sequence $\left(R_{n}\right)_{n}$ as soon as $R_{n}>R$. It then follows that the sequence $\left(\bar{u}_{n}, \bar{v}_{n}\right)_{n}$, the extensions by zero of $\left(u_{n}, v_{n}\right)$, is bounded in $H^{1}\left(\mathbb{R}^{N}\right) \times H^{1}\left(\mathbb{R}^{N}\right)$ and so, up to a subsequence, $\bar{u}_{n} \rightarrow u$ and $\bar{v}_{n} \rightarrow v$ weakly in $H^{1}\left(\mathbb{R}^{N}\right)$, with $I_{\lambda}^{\prime}(u, v)=0$. Using Fatou's lemma, we see that $I_{\lambda}(u, v) \leq \liminf I_{\lambda}\left(u_{n}, v_{n}\right)$.

We claim that $u \neq 0$. Indeed, suppose $u_{n} \rightarrow 0$ weakly in $H^{1}\left(\mathbb{R}^{N}\right)$. Then $\int_{\mathbb{R}^{N}} \frac{1}{1+\lambda|x|^{2}} f\left(u_{n}\right) v_{n} \rightarrow 0$. Since $I_{\lambda}^{\prime}\left(u_{n}, v_{n}\right)\left(v_{n}, 0\right) \rightarrow 0$, this implies that $v_{n} \rightarrow 0$ in $H^{1}\left(\mathbb{R}^{N}\right)$, and so $\int_{\mathbb{R}^{N}} g\left(v_{n}\right) u_{n} \rightarrow 0$. But then, since $I_{\lambda}^{\prime}\left(u_{n}, v_{n}\right)\left(0, u_{n}\right) \rightarrow 0$ we also conclude that $u_{n} \rightarrow 0$ in $H^{1}\left(\mathbb{R}^{N}\right)$. However, it follows readily from the identity $I_{\lambda}^{\prime}\left(u_{n}, v_{n}\right)\left(v_{n}, u_{n}\right)=0$ that there exists $\eta>0$ such that

$$
\left\|u_{n}\right\|_{H^{1}\left(\mathbb{R}^{N}\right)}+\left\|v_{n}\right\|_{H^{1}\left(\mathbb{R}^{N}\right)} \geq \eta .
$$


This contradiction shows that $u \neq 0$. Since $I_{\lambda}^{\prime}(u, v)=0$ and $(u, v) \neq(0,0)$ we have that $I_{\lambda}(u, v) \geq c_{0}^{\lambda}\left(\mathbb{R}^{N}\right)$, and this finishes the proof of Lemma 4.12.

Proof of Proposition 4.2. The existence of a ground state solution has been proved in 30. Let $(u, v) \in H^{1}\left(\mathbb{R}^{N}\right) \times H^{1}\left(\mathbb{R}^{N}\right)$ be such that $I^{\prime}(u, v)=0$ and $I(u, v)=$ $c_{0}\left(\mathbb{R}^{N}\right)$. By Proposition 4.6, $u$ lies in the space $E=W^{2, \frac{r+1}{r}}\left(\mathbb{R}^{N}\right) \cap W^{2, \frac{\alpha+1}{\alpha}}\left(\mathbb{R}^{N}\right)$ which was used in Lemma 4.5, and $J(u)=c_{0}\left(\mathbb{R}^{N}\right)$.

Let $w:=L^{-1}(|L u|) \in E$. We observe that $w>0$ and $w \geq|u|$. In the sequel, we use cutoff functions $\varphi_{R} \in \mathcal{D}\left(B_{R}(0)\right)$ such that $\varphi_{R}(x)=1$ for every $x \in B_{R / 2}(0)$ and $|\nabla \varphi(x)| \leq C / R$ for every $x \in \mathbb{R}^{N}$. For a fixed sequence $R \rightarrow \infty$, let $t_{R}>0$ be such that $J_{R}^{\prime}\left(t_{R} \varphi_{R} w\right)\left(t_{R} \varphi_{R} w\right)=0$. According to Lemmas 4.12 and 4.8 .

$$
c_{0}\left(\mathbb{R}^{N}\right) \leq c_{0}\left(B_{R}(0)\right) \leq J_{R}\left(t_{R} \varphi_{R} w\right)=J\left(t_{R} \varphi_{R} w\right) .
$$

In particular (cf. Lemma 4.5 $(a)$ ), the sequence $\left(t_{R}\right)$ is bounded and therefore, up to a subsequence, there exists $t_{0} \geq 0$ such that $t_{R} \rightarrow t_{0}$. Taking the limit as $R \rightarrow \infty$, we infer that

$$
c_{0}\left(\mathbb{R}^{N}\right) \leq J\left(t_{0} w\right),
$$

and in particular, $t_{0}>0$. Since $F$ and $H$ are even functions, we deduce that

$$
c_{0}\left(\mathbb{R}^{N}\right) \leq J\left(t_{0} u\right)+\int_{\mathbb{R}^{N}}\left(F\left(t_{0}|u|\right)-F\left(t_{0} w\right)\right) .
$$

Now, observe that, thanks to Lemma $4.5(b), \int_{\mathbb{R}^{N}}\left(F\left(t_{0}|u|\right)-F\left(t_{0} w\right)\right) \geq 0$. Since $w \geq|u|$, we may conclude as in the proof of Lemma 4.9 that $u v>0$ in $\mathbb{R}^{N}$.

We close this section by stating some results which will be used in section 5

Lemma 4.13. Consider the problem (1.4) with $p q>1$ satisfying (H1). The problem admits no sign changing strong solution $(u, v)$ such that $c_{0}\left(\mathbb{R}^{N}\right) \leq I(u, v)<$ $2 c_{0}\left(\mathbb{R}^{N}\right)$.

Proof. Let $(u, v)$ be a strong sign changing solution of (1.4). By Theorem 3.1, $J^{\prime}(u)=0, I(u, v)=J(u)$, and we recall from (3.1) and (2.8) that

$$
c_{0}\left(\mathbb{R}^{N}\right)=\frac{p q-1}{(p+1)(q+1)} \alpha_{p, q}^{\frac{p(q+1)}{p q-1}} .
$$

Let $u_{1}:=L^{-1}\left((L u)^{+}\right) \in W^{2, \frac{p+1}{p}}\left(\mathbb{R}^{N}\right), u_{2, \rho}:=-L^{-1}\left((L u)^{-}\right) \in W^{2, \frac{p+1}{p}}\left(\mathbb{R}^{N}\right)$, so that $u_{2, \rho} \leq 0 \leq u_{1}, u_{1, \rho}, u_{2, \rho} \neq 0, u=u_{1, \rho}+u_{2, \rho}$, and

$$
|u|^{q-1} u u_{i} \leq\left|u_{i}\right|^{q+1}, \quad i=1,2 .
$$

In particular,

$$
\alpha_{p, q}\left|u_{i}\right|_{q+1}^{\frac{p+1}{p}} \leq\left\|u_{i}\right\|^{\frac{p+1}{p}}=\int_{\mathbb{R}^{N}}|L u|^{\frac{1}{p}-1} L u L u_{i}=\int_{\mathbb{R}^{N}}|u|^{q-1} u u_{i} \leq \int_{\mathbb{R}^{N}}\left|u_{i}\right|^{q+1},
$$

that is,

$$
\left|u_{i}\right|_{q+1}^{\frac{p+1}{p}} \geq \alpha_{p, q}^{\frac{p+1}{p q-1}}, \quad i=1,2 .
$$


We then compute

$$
\begin{aligned}
I(u, v)=J(u) & =\frac{p}{p+1} \int_{\mathbb{R}^{N}}|L u|^{\frac{p+1}{p}}-\frac{1}{q+1} \int_{\mathbb{R}^{N}}|u|^{q+1} \\
& =\frac{p q-1}{(p+1)(q+1)} \|\left. u\right|^{\frac{p+1}{p}} \\
& =\frac{p q-1}{(p+1)(q+1)}\left(\left\|u_{1, \rho}\right\|^{\frac{p+1}{p}}+\left\|u_{2, \rho}\right\|^{\frac{p+1}{p}}\right) \\
& \geq \frac{p q-1}{(p+1)(q+1)} \alpha_{p, q}\left(\left|u_{1, \rho}\right|_{q+1}^{\frac{p+1}{p}}+\left|u_{2, \rho}\right|_{q+1}^{\frac{p+1}{p}}\right) \\
& \geq 2 \frac{p q-1}{(p+1)(q+1)} \alpha_{p, q} \alpha_{p, q}^{\frac{p+1}{p q-1}} \\
& =2 \frac{p q-1}{(p+1)(q+1)} \alpha_{p, q}^{\frac{p(q+1)}{p q-1}}=2 c_{0}\left(\mathbb{R}^{N}\right) .
\end{aligned}
$$

We state a compactness result for Palais-Smale sequences of the functional $I$. We first need a variant of Lemma 4.12, We use the same notation $I$ for the energy functional defined in the Sobolev space $H_{0}^{1}(\Omega) \times H_{0}^{1}(\Omega)$, where $\Omega$ is an exterior domain. Similarly, let $J$ be defined as in (4.7) in the space $W^{2, \frac{r+1}{r}}(\Omega) \cap W^{2, \frac{\alpha+1}{\alpha}}(\Omega) \cap$ $W_{0}^{1, \frac{r+1}{r}}(\Omega) \cap W_{0}^{1, \frac{\alpha+1}{\alpha}}(\Omega)$.

Lemma 4.14. We have that

$$
c_{0}\left(\mathbb{R}^{N}\right) \leq \inf \left\{I(u, v): u, v \in H_{0}^{1}(\Omega),(u, v) \neq(0,0), I^{\prime}(u, v)=0\right\} .
$$

Proof. Let us denote the right-hand side of the inequality by $c_{0}(\Omega)$. We may assume that $c_{0}(\Omega)$ is finite; that is, there exists $(u, v) \in H_{0}^{1}(\Omega) \times H_{0}^{1}(\Omega),(u, v) \neq(0,0)$, such that $I^{\prime}(u, v)=0$ and $I(u, v) \leq c_{0}(\Omega)+\varepsilon$, with $\varepsilon>0$ arbitrarily small. Since $I^{\prime}(u, v)=0$, we have that $u \in W^{2, \frac{r+1}{r}}(\Omega) \cap W^{2, \frac{\alpha+1}{\alpha}}(\Omega) \cap W_{0}^{1, \frac{r+1}{r}}(\Omega) \cap W_{0}^{1, \frac{\alpha+1}{\alpha}}(\Omega)$ and $I(u, v)=J(u)=\sup _{t>0} J(t u)$. We consider the ground state critical levels $c_{0}\left(B_{R}(0)\right)$ and $c_{0}\left(B_{R}(0) \cap \Omega\right)$. An inspection of the proof of Lemmas 4.84 .12 shows that $c_{0}\left(B_{R}(0)\right) \leq c_{0}\left(B_{R}(0) \cap \Omega\right)$ and $c_{0}\left(\mathbb{R}^{N}\right) \leq c_{0}\left(B_{R}(0) \cap \Omega\right)$ for every $R>0$.

We use the same cutoff functions $\varphi_{R} \in \mathcal{D}\left(B_{R}(0)\right)$ as in the proof of Theorem 4.1 above. For a fixed sequence $R \rightarrow \infty$, let $t_{R}>0$ be such that $J_{R}^{\prime}\left(t_{R} \varphi_{R} w\right)\left(t_{R} \varphi_{R} w\right)=$ 0 . Then

$$
c_{0}\left(\mathbb{R}^{N}\right) \leq c_{0}\left(B_{R}(0) \cap \Omega\right) \leq J_{R}\left(t_{R} \varphi_{R} u\right)=J\left(t_{R} \varphi_{R} u\right) .
$$

In particular, the sequence $\left(t_{R}\right)$ is bounded and so, up to a subsequence, there exists $t_{0} \geq 0$ such that $t_{R} \rightarrow t_{0}$. Taking the limit as $R \rightarrow \infty$, we conclude that

$$
c_{0}\left(\mathbb{R}^{N}\right) \leq J\left(t_{0} u\right) \leq J(u)=I(u, v) \leq c_{0}(\Omega)+\varepsilon,
$$

and we are done.

We keep using the notation $I$ for the energy functional defined in the symmetric Sobolev space $H_{0}^{1}(\Omega)^{G} \times H_{0}^{1}(\Omega)^{G}$. The number $\ell=\ell(G) \geq 1$ was defined in the Introduction. Our statement $(a)$ below will be used in the proof of Theorem 1.10 , while $(b)$ concerns the proof of Theorem 1.11. We denote by $P$ the positive cone $P:=\left\{u \in H^{1}\left(\mathbb{R}^{N}\right): u \geq 0\right\}$. 
Proposition 4.15. Consider the problem (1.4) with $p, q>1$ and $p(N-2)<N+2$, $q(N-2)<N+2$. Let $\left(u_{n}, v_{n}\right) \subset H_{0}^{1}(\Omega)^{G} \times H_{0}^{1}(\Omega)^{G}$ be a Palais-Smale sequence at level $c$, namely $I\left(u_{n}, v_{n}\right) \rightarrow c \in \mathbb{R}$ and $I^{\prime}\left(u_{n}, v_{n}\right) \rightarrow 0$.

(a) If $c<\ell c_{0}\left(\mathbb{R}^{N}\right)$, then, up to a subsequence, $\left(u_{n}, v_{n}\right)$ converges strongly to a critical point of $I$.

(b) If $c<(\ell+1) c_{0}\left(\mathbb{R}^{N}\right)$, then, up to a subsequence, either $\left(u_{n}, v_{n}\right)$ converges strongly to a critical point of $I$ or else $d\left(u_{n}+v_{n}, P \cup(-P)\right) \rightarrow 0$ as $n \rightarrow \infty$.

Proof. We use the characterization of the Palais-Smale sequences as given in [4, see also [39, by also taking the symmetry into account. Namely, since $c<2 \ell c_{0}\left(\mathbb{R}^{N}\right)$ we have that, for some $k$ points $g_{1}, \ldots, g_{k} \in G$, with $k \geq \ell$,

$$
\left(u_{n}, v_{n}\right)=\left(u_{0}, v_{0}\right)+\sum_{j=1}^{k}(u, v)\left(g_{j}^{-1} x-y^{(n)}\right)+\mathrm{o}(1) \quad \text { in } H^{1}\left(\mathbb{R}^{N}\right) \times H^{1}\left(\mathbb{R}^{N}\right),
$$

where $\left(u_{0}, v_{0}\right) \in H_{0}^{1}(\Omega)^{G} \times H_{0}^{1}(\Omega)^{G}, I^{\prime}\left(u_{0}, v_{0}\right)=0,(u, v) \in H^{1}\left(\mathbb{R}^{N}\right) \times H^{1}\left(\mathbb{R}^{N}\right)$, $I^{\prime}(u, v)=0$, and $\left(y^{(n)}\right)$ is an unbounded sequence in $\mathbb{R}^{N}$. Moreover, it follows that

$$
c=I\left(u_{0}, v_{0}\right)+k I(u, v),
$$

and either we have strong convergence $\left(u_{n}, v_{n}\right) \rightarrow\left(u_{0}, v_{0}\right)$ or else we can assert that $(u, v) \neq(0,0)$. From this the conclusion in $(a)$ follows immediately. As for $(b)$, assume that strong convergence does not hold. Then we have that $\left(u_{0}, v_{0}\right)=(0,0)$; otherwise, by Lemma 4.14, $c \geq c_{0}\left(\mathbb{R}^{N}\right)+k c_{0}\left(\mathbb{R}^{N}\right) \geq(1+\ell) c_{0}\left(\mathbb{R}^{N}\right)$, in contradiction with our assumption that $c<(\ell+1) c_{0}\left(\mathbb{R}^{N}\right)$. Now, since $\left(u_{0}, v_{0}\right)=(0,0)$, we have $c=k I(u, v) \geq \ell I(u, v)$. This yields $I(u, v)<(\ell+1) c_{0}\left(\mathbb{R}^{N}\right) / \ell \leq 2 c_{0}\left(\mathbb{R}^{N}\right)$, implying that $u+v$ does not change sign in $\mathbb{R}^{N}$ (cf. Lemma4.13). Since $u_{n}+v_{n}=$ $\sum_{j=1}^{k}(u+v)\left(g_{j}^{-1} x-y^{(n)}\right)+\mathrm{o}(1)$, the conclusion follows.

Remark 4.16. We have used several equivalent definitions of ground state solutions of (1.1) or (1.4). One could also have used the dual variational principle of Clarke and Ekeland; see e.g. 12 for the application of the method to our framework. It is worth pointing out that Alves et al. 1] have proved, in the case of the system (1.4) with $p, q>1$, that any critical point of the dual action at the least energy level (of the dual action) leads to a solution $(u, v)$ of the system (1.4) such that either $\left(u^{+}, v^{+}\right)=(0,0)$ or $\left(u^{-}, v^{-}\right)=(0,0)$. This brings another way of proving that the ground state solutions of (1.4) satisfy $\left(u^{+}, v^{+}\right)=(0,0)$ or $\left(u^{-}, v^{-}\right)=(0,0)$. Indeed, one can easily show that the critical points of the dual action at the least energy level give rise to ground state solutions of (1.4) in the sense previously defined and vice versa. However, the duality method requires $p, q>1$ while Theorem 1.9 applies under the assumption $p q>1$. The duality method can also be used to treat (1.1) in a bounded domain.

\section{THE CASE OF AN EXTERIOR DOMAIN}

This section is devoted to the proof of Theorems 1.10 and 1.11. As in the preceding section, we will introduce a further degree of generalization, and we consider a more general system

$$
\left\{\begin{aligned}
-\Delta u+u & =g(v) & & \text { in } \Omega \\
-\Delta v+v & =f(u) & & \text { in } \Omega, \\
u, v & =0 & & \text { on } \partial \Omega
\end{aligned}\right.
$$


with $\Omega=\mathbb{R}^{N} \backslash \omega$, where $\omega$ is a smooth and bounded domain of $\mathbb{R}^{N}, N \geq 3$. Both functions $f$ and $g$ are assumed to be of class $C^{1}$ and satisfy the following set of assumptions:

(fg1) $f(0)=g(0)=f^{\prime}(0)=g^{\prime}(0)=0$;

(fg2) there exist $p, q>1$ satisfying (H1) such that

$$
|f(s)| \leq C\left(1+|s|^{q}\right) \text { and }|g(s)| \leq C\left(1+|s|^{p}\right) ;
$$

(fg3) there exists $\delta^{\prime}>0$ such that

$$
0<\left(1+\delta^{\prime}\right) f(s) s \leq f^{\prime}(s) s^{2} \text { and } 0<\left(1+\delta^{\prime}\right) g(s) s \leq g^{\prime}(s) s^{2} ;
$$

(fg4) $f$ and $g$ are odd symmetric functions;

(fg5) $f^{\prime}(s)$ and $g^{\prime}(s)$ are non-decreasing functions for $s>0$;

(fg6) there exists $C_{0}>0$ such that for every $s \in \mathbb{R}$,

$$
f^{\prime}(2 s) s^{2} \leq C_{0} f(s) s \text { and } g^{\prime}(2 s) s^{2} \leq C_{0} g(s) s .
$$

We stress that it is useful for us to allow more general non-linear terms in the system since, even in the case when we start from a system with pure power nonlinear terms, we end up working with systems having non-pure power ones; see the modified system $\left(S_{n}\right)$ and Lemma 4.3. In particular (see also Remark 4.4), besides (fg1)-(fg6), in the sequel we assume that (fg2') holds. Before turning to the complete proofs of Theorems 1.10 and 1.11, which will be worked out in subsection 5.2. we establish some crucial estimates. These are elementary but at some points specific to the fact that we are dealing with a system rather than a single equation. We will present them in detail, so as to insure ourselves that they are independent of the truncation procedure performed to derive Theorem 4.1 from Proposition 4.2 .

\subsection{Some technical estimates.}

Lemma 5.1. There exist $\mu>1$ and $C_{1}>0$ such that for every $t, a \geq 0$,

$$
\frac{\mu}{2}(f(t) a+f(a) t) \leq F(a+t)-F(t)-F(a) \leq C_{1}(f(t) a+f(a) t) .
$$

Proof. Take $\mu=\frac{2\left(1+\delta^{\prime}\right)}{2+\delta^{\prime}}$, where $\delta^{\prime}$ is given by (fg3). Hence, for every $s \in \mathbb{R}$, we have

$$
f^{\prime}(s) s^{2} \geq \frac{\mu}{2-\mu} f(s) s .
$$

Define $\theta_{a}: \mathbb{R} \rightarrow \mathbb{R}$ by

$$
\theta_{a}(t)=F(a+t)-F(t)-F(a)-\frac{\mu}{2}(f(t) a+f(a) t) .
$$

We may assume without loss of generality that $t \geq a$. Then, since $f^{\prime}$ is nondecreasing, we infer that

$$
\begin{aligned}
\theta_{a}^{\prime}(t) & =f(a+t)-f(t)-\frac{\mu}{2} f^{\prime}(t) a-\frac{\mu}{2} f(a) \geq f^{\prime}(t) a-\frac{\mu}{2} f^{\prime}(t) a-\frac{\mu}{2} f(a) \\
& =\frac{2-\mu}{2} f^{\prime}(t) a-\frac{\mu}{2} f(a) \geq \frac{2-\mu}{2} f^{\prime}(a) a-\frac{\mu}{2} f(a) \geq 0 .
\end{aligned}
$$

In order to prove the inequality on the left-hand side of (5.2), it is then sufficient to show that $\theta_{a}(a) \geq 0$ for all $a \geq 0$, that is,

$$
\Theta(a):=\theta_{a}(a)=F(2 a)-2 F(a)-\mu f(a) a \geq 0 .
$$


Now, since $\Theta(0)=0$ and

$$
\begin{aligned}
\Theta^{\prime}(s) & =2 f(2 s)-2 f(s)-\mu s f^{\prime}(s)-\mu f(s) \\
& \geq 2 s f^{\prime}(s)-\mu s f^{\prime}(s)-\mu f(s)=(2-\mu) s f^{\prime}(s)-\mu f(s) \geq 0,
\end{aligned}
$$

the left inequality in the statement of Lemma 5.1 follows. On the other hand, we deduce from (fg3) and (fg6) that for all $s \geq 0, f(2 s) \leq C_{1} f(s)$, where $C_{1}=$ $2 C_{0} /\left(1+\delta^{\prime}\right)$. This yields

$$
F(a+t)-F(t)-F(a) \leq F(a+t)-F(t) \leq f(a+t) a \leq f(2 t) a \leq C_{1} f(t) a .
$$

Lemma 5.2. For every $t, a \geq 0$, we have

$$
-f(t) a-f(a) t \leq F(t-a)-F(t)-F(a) \leq 0 .
$$

Moreover, for every $R>0$, there exists $C>0$ such that

$$
(f(t-a)-f(t)+f(a))^{2} \leq C(f(a) t+f(t) a)
$$

for $0 \leq a, t \leq R$.

Proof. We define $\eta_{a}: \mathbb{R} \rightarrow \mathbb{R}$ by $\eta_{a}(t)=F(t-a)-F(t)-F(a)+f(t) a+f(a) t$. As for the first two inequalities, since $F$ is even we may assume $t \geq a$. We observe that $f(t) t \geq F(t)$ since $f^{\prime} \geq 0$. It follows that $\eta_{a}(a)=-2 F(a)+2 f(a) a \geq 0$. On the other hand, for some $\xi \in] t-a, t[$, we have

$$
\eta_{a}^{\prime}(t)=f(t-a)-f(t)+f^{\prime}(t) a+f(a)=\left(f^{\prime}(t)-f^{\prime}(\xi)\right) a+f(a) \geq f(a) \geq 0,
$$

and this shows that $\eta_{a}(t) \geq 0$. Since $0 \leq t-a \leq t$, we also have

$$
F(t-a)-F(t)-F(a) \leq F(t-a)-F(t) \leq 0 .
$$

Concerning the final statement, since $f$ is odd we may assume that $\zeta_{a}$ defined by $\zeta_{a}(t)=f(t-a)-f(t)+f(a)$ is non-negative. In particular, we must have $t \leq a$, since $\zeta_{a}(a)=0, \zeta_{a}^{\prime}(a)=-f^{\prime}(a)<0$, and $\zeta_{a}^{\prime}(s)=f^{\prime}(s-a)-f^{\prime}(s) \leq 0$ for all $s \geq a$. Thus, for some $\xi \in] a-t, a[$, we have

$$
0 \leq \zeta_{a}(t)=f(a)-f(a-t)-f(t)=f^{\prime}(\xi) t-f(t) \leq f^{\prime}(a) t-f(t) \leq f^{\prime}(a) t .
$$

It follows from (fg5) $-(\mathrm{fg} 6)$ that $f^{\prime}(s) s \leq C_{0} f(s)$ for every $s \in \mathbb{R}$. Thus, by taking squares, we deduce the existence of $C_{R}>0$ such that

$$
\zeta_{a}^{2}(t) \leq C_{R} t^{2} f^{\prime}(a) \leq C_{R} t a f^{\prime}(a) \leq C_{R} C_{0} t f(a),
$$

for $0 \leq a, t \leq R$. This completes the proof.

Now, let us fix a positive ground state solution $(u, v)$ of the system

$$
-\Delta u+u=g(v), \quad-\Delta v+v=f(u), \quad u, v \in C^{2}\left(\mathbb{R}^{N}\right) \cap H^{1}\left(\mathbb{R}^{N}\right) ;
$$

that is, $I(u, v)=c_{0}\left(\mathbb{R}^{N}\right)$ and $u>0, v>0$ in $\mathbb{R}^{N}$. According to [6, Theorem 2] we can assume that both $u$ and $v$ are radially symmetric with respect to the origin. By [17, Theorem 2.2], both $u$ and $v$ decay exponentially to zero. In fact, since we deal with positive solutions of the system, by classical arguments it can be shown that for every $\varepsilon>0$ there exist $c_{1}, c_{2}>0$ such that for every $x \in \mathbb{R}^{N}$,

$$
c_{1} e^{-(1+\varepsilon)|x|} \leq u(x) \leq c_{2} e^{-(1-\varepsilon)|x|}
$$

and similarly for $v(x)$ (see e.g. [28, Lemma 3.3 and Lemma 3.4]). 
Let $y_{1}, y_{2} \in \mathbb{R}^{N}$ be such that $\left|y_{1}\right|=\left|y_{2}\right|=1,\left|y_{1}-y_{2}\right|>0$, and define $u_{1, \rho}, u_{2, \rho}, v_{1, \rho}, v_{2, \rho}$ by $u_{i, \rho}(x)=u\left(x-\rho y_{i}\right), v_{i, \rho}(x)=v\left(x-\rho y_{i}\right)$, where $i=1,2$ and $\rho>0$. Since

$$
I\left(u_{1, \rho}+u_{2, \rho}, v_{1, \rho}+v_{2, \rho}\right)=2 I(u, v)+\gamma_{f}\left(u_{1, \rho}, u_{2, \rho}\right)+\gamma_{g}\left(v_{1, \rho}, v_{2, \rho}\right),
$$

where

$$
\begin{aligned}
\gamma_{f}\left(u_{1, \rho}, u_{2, \rho}\right):=\frac{1}{2} \int_{\mathbb{R}^{N}}\left(f\left(u_{1, \rho}\right) u_{2, \rho}\right. & \left.+f\left(u_{2, \rho}\right) u_{1, \rho}\right) \\
& -\int_{\mathbb{R}^{N}}\left(F\left(u_{1, \rho}+u_{2, \rho}\right)-F\left(u_{1, \rho}\right)-F\left(u_{2, \rho}\right)\right)
\end{aligned}
$$

and similarly for $\gamma_{g}\left(v_{1, \rho}, v_{2, \rho}\right)$, we deduce from Lemma 5.1 that

$$
\begin{aligned}
& I\left(u_{1, \rho}+u_{2, \rho}, v_{1, \rho}+v_{2, \rho}\right) \leq 2 I(u, v) \\
& \quad-\delta_{0} \int_{\mathbb{R}^{N}}\left(F\left(u_{1, \rho}+u_{2, \rho}\right)-F\left(u_{1, \rho}\right)-F\left(u_{2, \rho}\right)\right) \\
& \quad-\delta_{0} \int_{\mathbb{R}^{N}}\left(G\left(v_{1, \rho}+v_{2, \rho}\right)-G\left(v_{1, \rho}\right)-G\left(v_{2, \rho}\right)\right),
\end{aligned}
$$

for some $\delta_{0}>0$. In particular, we infer that

$$
I\left(u_{1, \rho}+u_{2, \rho}, v_{1, \rho}+v_{2, \rho}\right)<2 I(u, v) .
$$

This is the basic estimate which is required in the case of a single equation (cf. [28, Lemma 6]). However, in order to deal with the system, we need a further estimate (see the proof of Lemma 5.5 below).

Lemma 5.3. Given $\delta>0$ we have, for every $x \in \mathbb{R}^{N}$ and every sufficiently large $\rho$,

$$
\left(f\left(u_{1, \rho}+u_{2, \rho}\right)-f\left(u_{1, \rho}\right)-f\left(u_{2, \rho}\right)\right)^{2} \leq \delta\left(F\left(u_{1, \rho}+u_{2, \rho}\right)-F\left(u_{1, \rho}\right)-F\left(u_{2, \rho}\right)\right) .
$$

Proof. Since $\lim _{|x| \rightarrow \infty} u(x)=0$, for every $\nu>0$ there exists $\rho_{0}>0$ such that, for every $x \in \mathbb{R}^{N}$ and every $\rho \geq \rho_{0}$, either $u_{1, \rho}(x) \leq \nu$ or $u_{2, \rho}(x) \leq \nu$. In particular, $\forall \nu>0 \exists \rho_{0}>0: \forall x \in \mathbb{R}^{N}, \rho \geq \rho_{0}$, either $u_{2, \rho}(x) \leq \nu$ or $u_{1, \rho}(x) \leq \nu u_{2, \rho}(x)$. In order to prove the lemma we can assume without loss of generality that, at a given point $x, u_{1, \rho} \leq u_{2, \rho}$. Next we observe that

$$
0 \leq f\left(u_{1, \rho}+u_{2, \rho}\right)-f\left(u_{1, \rho}\right)-f\left(u_{2, \rho}\right) \leq f\left(u_{1, \rho}+u_{2, \rho}\right)-f\left(u_{2, \rho}\right)
$$

and that, according to Lemma 5.1 .

$$
F\left(u_{1, \rho}+u_{2, \rho}\right)-F\left(u_{1, \rho}\right)-F\left(u_{2, \rho}\right) \geq \frac{\mu}{2} f\left(u_{2, \rho}\right) u_{1, \rho}
$$

for some $\mu>0$. It will thus be sufficient to show that, for sufficiently large $\rho>0$,

$$
\left(f\left(u_{1, \rho}+u_{2, \rho}\right)-f\left(u_{2, \rho}\right)\right)^{2} \leq \delta f\left(u_{2, \rho}\right) u_{1, \rho}, \quad \text { with } u_{1, \rho} \leq u_{2, \rho} .
$$

Since $f\left(u_{1, \rho}+u_{2, \rho}\right)-f\left(u_{2, \rho}\right) \leq f^{\prime}\left(2 u_{2, \rho}\right) u_{1, \rho}$, it is sufficient to show that

$$
\left(f^{\prime}\right)^{2}\left(2 u_{2, \rho}\right) u_{1, \rho} \leq \delta f\left(u_{2, \rho}\right)
$$

According to our previous remark, we can restrict our attention to the following two cases. Assuming first that case $u_{2, \rho} \leq \nu$ for a small $\nu>0$, then, thanks to (fg6), we conclude, since $f^{\prime}(0)=0$, that

$$
\left(f^{\prime}\right)^{2}\left(2 u_{2, \rho}\right) u_{1, \rho} \leq\left(f^{\prime}\right)^{2}\left(2 u_{2, \rho}\right) u_{2, \rho} \leq C_{0} f^{\prime}\left(2 u_{2, \rho}\right) f\left(u_{2, \rho}\right) \leq \delta f\left(u_{2, \rho}\right) .
$$


In the case that we have $u_{1, \rho} \leq \nu u_{2, \rho}$, then the fact that $\left\|u_{2, \rho}\right\|_{L^{\infty}\left(\mathbb{R}^{N}\right)}<\infty$ leads to

$$
\left(f^{\prime}\right)^{2}\left(2 u_{2, \rho}\right) u_{1, \rho} \leq \nu\left(f^{\prime}\right)^{2}\left(2 u_{2, \rho}\right) u_{2, \rho} \leq \nu C_{0} f^{\prime}\left(2 u_{2, \rho}\right) f\left(u_{2, \rho}\right) \leq \delta f\left(u_{2, \rho}\right) .
$$

This completes the proof of the lemma.

In the next lemma, $(u, v)$ is still a ground state solution of (4.1) and therefore of $\left(S_{n}\right)$ for $n$ large enough. We keep the notation $u_{i, \rho}(x)=u\left(x-\rho y_{i}\right), v_{i, \rho}(x)=$ $v\left(x-\rho y_{i}\right)$.

Lemma 5.4. For every $\rho>0$, the quantity

$$
\sup \left\{I\left(t\left(u_{1, \rho}+u_{2, \rho}\right)+\psi, t\left(v_{1, \rho}+v_{2, \rho}\right)-\psi\right): t \geq 0, \psi \in H^{1}\left(\mathbb{R}^{N}\right)\right\}
$$

is bounded from above by

$$
I\left(u_{1, \rho}+u_{2, \rho}, v_{1, \rho}+v_{2, \rho}\right)+C \nu(\rho)^{2},
$$

where

$$
\nu(\rho)=\sup \left\{I^{\prime}\left(u_{1, \rho}+u_{2, \rho}, v_{1, \rho}+v_{2, \rho}\right)(\varphi, \psi):\|\varphi\|_{H^{1}\left(\mathbb{R}^{N}\right)}+\|\psi\|_{H^{1}\left(\mathbb{R}^{N}\right)} \leq 1\right\} .
$$

The constant $C$ is independent of $\rho$ as $\rho \rightarrow \infty$, and also of the (sufficiently large) truncation procedure in Proposition 4.2 .

Proof. We adopt the notation $u_{\rho}=u_{1, \rho}+u_{2, \rho}, v_{\rho}=v_{1, \rho}+v_{2, \rho}$, and observe that these are bounded sequences in $H^{1}\left(\mathbb{R}^{N}\right) \cap L^{\infty}\left(\mathbb{R}^{N}\right)$ as $\rho \rightarrow \infty$. We claim that

$$
I\left(t u_{\rho}+\psi, t v_{\rho}-\psi\right) \rightarrow-\infty \text { as } t \rightarrow \infty,
$$

uniformly in $\psi \in H^{1}\left(\mathbb{R}^{N}\right)$ and $n$ large. Indeed, we recall that there exist some $\theta>2$ and $C>0$ independent of $n$ such that

$$
f_{n}(s) s \geq C|s|^{\theta} \text { and } g_{n}(s) s \geq C|s|^{\theta},
$$

for $|s| \geq 1$. Then, as shown in [29, Lemma 2.1], if our claim does not hold, we infer that $\int_{\mathbb{R}^{N}}\left(u_{\rho}+v_{\rho}\right)^{\theta} \rightarrow 0$ for some sequence $\rho \rightarrow \infty$. However, we have

$$
\liminf _{\rho \rightarrow \infty}\left\|u_{\rho}+v_{\rho}\right\|_{L^{\theta}\left(\mathbb{R}^{N}\right)} \geq\|u\|_{L^{\theta}\left(\mathbb{R}^{N}\right)}>0
$$

which is a contradiction. This proves our claim and shows that the supremum is attained at some $I\left(t_{\rho, n} u_{\rho}+\psi_{\rho, n}, t_{\rho, n} v_{\rho}-\psi_{\rho, n}\right)$ with $t_{\rho, n}$ positive and bounded, and $\psi_{\rho, n} \in H^{1}\left(\mathbb{R}^{N}\right)$. Since

$$
I\left(t_{\rho, n} u_{\rho}+\psi, t_{\rho, n} v_{\rho}-\psi\right) \leq\left\langle t_{\rho, n} u_{\rho}+\psi, t_{\rho, n} v_{\rho}-\psi\right\rangle \rightarrow-\infty
$$

as $\|\psi\|_{H^{1}\left(\mathbb{R}^{N}\right)} \rightarrow \infty$, where $\langle$,$\rangle stands for the usual inner product of H^{1}\left(\mathbb{R}^{N}\right)$, the sequence $\left(\psi_{\rho, n}\right)$ is bounded in $H^{1}\left(\mathbb{R}^{N}\right)$.

Now, the optimality of $\psi_{\rho, n}$ implies that for all $\phi \in H^{1}\left(\mathbb{R}^{N}\right)$,

$$
I^{\prime}\left(t_{\rho, n} u_{\rho}+\psi_{\rho, n}, t_{\rho, n} v_{\rho}-\psi_{\rho, n}\right)(\phi,-\phi)=0 .
$$

In particular, $\psi_{\rho, n}$ is the (unique) solution in $H^{1}\left(\mathbb{R}^{N}\right)$ of

$$
-2 \Delta \psi_{\rho, n}+2 \psi_{\rho, n}+a_{\rho, n}\left(x, \psi_{\rho, n}\right) \psi_{\rho, n}=b_{\rho, n}(x),
$$

where $b_{\rho, n}=g_{n}\left(t_{\rho, n} v_{\rho}\right)-f_{n}\left(t_{\rho, n} u_{\rho}\right)-t_{\rho, n} \Delta\left(v_{\rho}-u_{\rho}\right)$ and

$$
\begin{aligned}
a_{\rho, n}(x, s) & =\frac{g_{n}\left(t_{\rho, n} v_{\rho}(x)\right)-g_{n}\left(t_{\rho, n} v_{\rho}(x)-s\right)}{s}+\frac{f_{n}\left(t_{\rho, n} u_{\rho}(x)+s\right)-f_{n}\left(t_{\rho, n} u_{\rho}(x)\right)}{s} \\
& \geq 0 .
\end{aligned}
$$


Since $a_{\rho, n}(x, s) \geq 0$, we have $\left|\psi_{\rho, n}\right| \leq \phi_{\rho, n}$ in $\mathbb{R}^{N}$, where $\phi_{\rho, n}$ is the unique solution in $H^{1}\left(\mathbb{R}^{N}\right)$ of

$$
-2 \Delta \phi_{\rho, n}+2 \phi_{\rho, n}=\left|b_{\rho, n}(x)\right| .
$$

Since $b_{n, \rho}$ is bounded in every $L^{s}\left(\mathbb{R}^{N}\right)$ space independently of $\rho$ and $n$, by elliptic regularity we deduce that $\phi_{\rho, n}$ is bounded in $L^{\infty}\left(\mathbb{R}^{N}\right)$ independently of $\rho$ and $n$, and so is $\psi_{\rho, n}$.

At last, we conclude that the supremum is given by $I\left(t_{\rho, n} u_{\rho}+\psi_{\rho, n}, t_{\rho . n} v_{\rho}-\psi_{\rho, n}\right)$ with $\left\|t_{\rho . n} u_{\rho}+\psi_{\rho, n}\right\|_{L^{\infty}\left(\mathbb{R}^{N}\right)}$ and $\left\|t_{\rho, n} v_{\rho}-\psi_{\rho, n}\right\|_{L^{\infty}\left(\mathbb{R}^{N}\right)}$ bounded independently of $\rho$ and $n$, and of the truncations $f_{n}$ and $g_{n}$ for $n$ large. The conclusion then follows directly by [30, Proposition 2.5].

In the sequel, we drop the explicit dependence of $f$ and $g$ with respect to $n$. When necessary, we point out the independence of the estimates with respect to the truncations.

Lemma 5.5. There exists $\rho_{0}>0$ such that, for every $\rho \geq \rho_{0}$,

$$
\sup \left\{I\left(t\left(u_{1, \rho}+u_{2, \rho}\right)+\psi, t\left(v_{1, \rho}+v_{2, \rho}\right)-\psi\right): t \geq 0, \psi \in H^{1}\left(\mathbb{R}^{N}\right)\right\}<2 I(u, v) .
$$

Proof. According to Lemma 5.4 we may focus on estimates of the quantities $\nu(\rho)^{2}$ and $I\left(u_{1, \rho}+u_{2, \rho}, v_{1, \rho}+v_{2, \rho}\right)$ to reach the conclusion. It can be checked that, for every $\varphi \in H^{1}\left(\mathbb{R}^{N}\right)$,

$$
I^{\prime}\left(u_{1, \rho}+u_{2, \rho}, v_{1, \rho}+v_{2, \rho}\right)(\varphi, 0)=\int_{\mathbb{R}^{N}}\left(f\left(u_{1, \rho}\right)+f\left(u_{2, \rho}\right)-f\left(u_{1, \rho}+u_{2, \rho}\right)\right) \varphi,
$$

and similarly for $I^{\prime}\left(u_{1, \rho}+u_{2, \rho}, v_{1, \rho}+v_{2, \rho}\right)(0, \psi)$. We apply the Cauchy-Schwarz inequality and use Lemma 5.3 , by recalling the previous definitions of $\gamma_{f}\left(u_{1, \rho}, u_{2, \rho}\right)$ and $\gamma_{g}\left(v_{1, \rho}, v_{2, \rho}\right)$, we conclude that

$$
\begin{aligned}
& I\left(u_{1, \rho}+u_{2, \rho}, v_{1, \rho}+v_{2, \rho}\right)+C \nu(\rho)^{2} \leq 2 I(u, v) \\
& \quad-\delta\left(\int_{\mathbb{R}^{N}} f\left(u_{1, \rho}\right) u_{2, \rho}+f\left(u_{2, \rho}\right) u_{1, \rho}+g\left(v_{1, \rho}\right) v_{2, \rho}+g\left(v_{2, \rho}\right) v_{1, \rho}\right),
\end{aligned}
$$

for some small $\delta>0$ and every sufficiently large $\rho$. The conclusion follows.

Now, let $\xi_{R} \in C^{\infty}\left(\mathbb{R}^{N} ; \mathbb{R}\right)$ be such that $\xi_{R}=0$ in $B_{R}(0)$ and $\xi_{R}=1$ in $\mathbb{R}^{N} \backslash B_{2 R}(0)$, where $R>0$ is such that $\mathbb{R}^{N} \backslash \Omega \subset B_{R}(0)$.

Lemma 5.6. Assume $\left|y_{1}-y_{2}\right|<2$. There exists $\rho_{0}>0$ such that, for every $\rho \geq \rho_{0}$,

$$
\sup \left\{I\left(t\left(u_{1, \rho}+u_{2, \rho}\right) \xi_{R}+\psi, t\left(v_{1, \rho}+v_{2, \rho}\right) \xi_{R}-\psi\right): t \geq 0, \psi \in H_{0}^{1}(\Omega)\right\}<2 I(u, v) .
$$

Proof. As in the proof of Lemma 5.5. we estimate $I\left(\left(u_{1, \rho}+u_{2, \rho}\right) \xi_{R},\left(v_{1, \rho}+v_{2, \rho}\right) \xi_{R}\right)+$ $C \nu(\rho, R)^{2}$, where $\nu(\rho, R)$ is given by

$$
\sup \left\{I^{\prime}\left(\left(u_{1, \rho}+u_{2, \rho}\right) \xi_{R},\left(v_{1, \rho}+v_{2, \rho}\right) \xi_{R}\right)(\varphi, \psi):\|\varphi\|_{H^{1}\left(\mathbb{R}^{N}\right)}+\|\psi\|_{H^{1}\left(\mathbb{R}^{N}\right)} \leq 1\right\} .
$$

It is easy to see that

$$
\begin{aligned}
& I\left(\left(u_{1, \rho}+u_{2, \rho}\right) \xi_{R},\left(v_{1, \rho}+v_{2, \rho}\right) \xi_{R}\right)+\nu(\rho, R)^{2} \\
& \quad \leq I\left(u_{1, \rho}+u_{2, \rho}, v_{1, \rho}+v_{2, \rho}\right)+C \nu(\rho)^{2}+C \int_{|x| \leq 3 R}\left(u_{1, \rho}^{2}+u_{2, \rho}^{2}+v_{1, \rho}^{2}+v_{2, \rho}^{2}\right) .
\end{aligned}
$$


In view of the estimates in the proof of Lemma 5.5, in order to prove Lemma 5.6 it is therefore sufficient to show that, given $\delta>0$, there exists $\rho_{0}>0$ such that, for every $\rho \geq \rho_{0}$,

$$
\int_{|x| \leq 3 R}\left(u_{1, \rho}^{2}+u_{2, \rho}^{2}+v_{1, \rho}^{2}+v_{2, \rho}^{2}\right) \leq \delta \int_{\mathbb{R}^{N}} f\left(u_{1, \rho}\right) u_{2, \rho}
$$

Let us estimate the first integral, the remaining ones being estimated in a similar way. We use the asymptotic behavior

$$
c_{1} e^{-(1+\varepsilon)|x|} \leq u(x) \leq c_{2} e^{-(1-\varepsilon)|x|} .
$$

For a small $\varepsilon>0$, we have

$$
\int_{\mathbb{R}^{N}} f\left(u_{1, \rho}\right) u_{2, \rho} \geq c_{1}^{\prime} e^{-\rho\left|y_{1}-y_{2}\right|(1+\varepsilon)},
$$

while $\int_{\mathbb{R}^{N}} u_{1, \rho}^{2} \leq c_{2}^{\prime} e^{-2 \rho\left|y_{1}\right|(1-\varepsilon)}$. The conclusion follows from the assumption that $2\left|y_{1}\right|=2>\left|y_{1}-y_{2}\right|$.

Lemma 5.7. Assume $\left|y_{1}-y_{2}\right|<1$. Then, given $\delta>0$ we have, for every sufficiently large $\rho$,

$\int_{\mathbb{R}^{N}}\left(f\left(u_{1, \rho}+u_{2, \rho}\right) u+f(u)\left(u_{1, \rho}+u_{2, \rho}\right)\right) \leq \delta \int_{\mathbb{R}^{N}}\left(F\left(u_{1, \rho}+u_{2, \rho}\right)-F\left(u_{1, \rho}\right)-F\left(u_{2, \rho}\right)\right)$.

Proof. It follows from (fg3) and (fg6) that $f(2 s) \leq C_{1} f(s)$ for $s \geq 0$, where $C_{1}=$ $2 C_{0} /\left(1+\delta^{\prime}\right)$. As a consequence, we infer that

$$
\begin{aligned}
& \int_{\mathbb{R}^{N}} f\left(u_{1, \rho}+u_{2, \rho}\right) u \leq \int_{\mathbb{R}^{N}}(\left.f\left(2 u_{1, \rho}\right) u+f\left(2 u_{2, \rho}\right) u\right) \\
& \leq C_{1} \int_{\mathbb{R}^{N}}\left(f\left(u_{1, \rho}\right) u+f\left(u_{2, \rho}\right) u\right)=2 C_{1} \int_{\mathbb{R}} f\left(u_{1, \rho}\right) u,
\end{aligned}
$$

where in the last inequality we have used the fact that $u$ is radially symmetric and $\left|y_{1}\right|=\left|y_{2}\right|=1$. Thus, thanks also to Lemma 5.1, it is sufficient to prove that

$$
\int_{\mathbb{R}^{N}} f\left(u_{1, \rho}\right) u+\int_{\mathbb{R}^{N}} f(u) u_{1, \rho} \leq \delta \int_{\mathbb{R}^{N}} f\left(u_{1, \rho}\right) u_{2, \rho},
$$

for $\rho$ sufficiently large. We again use the asymptotic behavior (5.3). For a small $\varepsilon>0$, we have

$$
\int_{\mathbb{R}^{N}} f\left(u_{1, \rho}\right) u_{2, \rho} \geq c_{1}^{\prime} e^{-\rho\left|y_{1}-y_{2}\right|(1+\varepsilon)},
$$

while, since $\int_{\mathbb{R}^{N}} f(u(x)) e^{(1-\varepsilon)|x|} d x<\infty$, the two integrals on the left-hand side above are dominated by $c_{2}^{\prime} e^{-\rho\left|y_{1}\right|(1-\varepsilon)}$. The conclusion follows from the assumption that $\left|y_{1}\right|>\left|y_{1}-y_{2}\right|$.

Lemma 5.8. Assume $\left|y_{1}-y_{2}\right|<1$. There exists $\rho_{0}>0$ such that, for every $\rho \geq \rho_{0}$, we have

$$
\begin{aligned}
\sup \left\{I\left(s\left(u_{1, \rho}+u_{2, \rho}\right)-t u+\psi, s\left(v_{1, \rho}+v_{2, \rho}\right)-t v-\psi\right): t, s \geq 0, \psi\right. & \left.\in H^{1}\left(\mathbb{R}^{N}\right)\right\} \\
& <3 I(u, v) .
\end{aligned}
$$

Proof. For the sake of clarity, we split the proof into three steps. 
Step 1. It can be proved along the same lines of [30, Section 2] that the left-hand side above is dominated by

$$
I\left(u_{1, \rho}+u_{2, \rho}-u, v_{1, \rho}+v_{2, \rho}-v\right)+C \widetilde{\nu}(\rho)^{2},
$$

where

$\widetilde{\nu}(\rho)=\sup \left\{I^{\prime}\left(u_{1, \rho}+u_{2, \rho}-u, v_{1, \rho}+v_{2, \rho}-v\right)(\varphi, \psi):\|\varphi\|_{H^{1}\left(\mathbb{R}^{N}\right)}+\|\psi\|_{H^{1}\left(\mathbb{R}^{N}\right)} \leq 1\right\}$.

The constant $C$ is independent of $\rho$ as $\rho \rightarrow \infty$, and also of the truncations. Since the details are a bit technical, we present them in the Appendix.

Step 2. At first we estimate

$$
\begin{aligned}
& I\left(u_{1, \rho}+u_{2, \rho}-u, v_{1, \rho}+v_{2, \rho}-v\right) \\
& \quad \leq I\left(u_{1, \rho}+u_{2, \rho}, v_{1, \rho}+v_{2, \rho}\right)+I(u, v)+\gamma_{f}\left(u_{1, \rho}, u_{2, \rho}, u\right)+\gamma_{g}\left(v_{1, \rho}, v_{2, \rho}, v\right),
\end{aligned}
$$

where

$$
\gamma_{f}\left(u_{1, \rho}, u_{2, \rho}, u\right):=\int_{\mathbb{R}^{N}}\left(F\left(u_{1, \rho}+u_{2, \rho}\right)+F(u)-F\left(u_{1, \rho}+u_{2, \rho}-u\right)\right)
$$

and similarly for $\gamma_{g}\left(v_{1, \rho}, v_{2, \rho}, v\right)$. We have seen in the proof of Lemma 5.5 that

$$
I\left(u_{1, \rho}+u_{2, \rho}, v_{1, \rho}+v_{2, \rho}\right) \leq 2 I(u, v)-3 \delta \int_{\mathbb{R}^{N}}\left(F\left(u_{1, \rho}+u_{2, \rho}\right)-F\left(u_{1, \rho}\right)-F\left(u_{2, \rho}\right)\right)
$$

for some small $\delta>0$, while Lemma 5.2 implies that

$$
\gamma_{f}\left(u_{1, \rho}, u_{2, \rho}, u\right) \leq \int_{\mathbb{R}^{N}}\left(f\left(u_{1, \rho}+u_{2, \rho}\right) u+f(u)\left(u_{1, \rho}+u_{2, \rho}\right)\right) .
$$

It follows then from Lemma 5.7 that

$$
\begin{aligned}
& I\left(u_{1, \rho}+u_{2, \rho}-u, v_{1, \rho}+v_{2, \rho}-v\right) \\
& \leq 3 I(u, v)-2 \delta \int_{\mathbb{R}^{N}}\left(F\left(u_{1, \rho}+u_{2, \rho}\right)-F\left(u_{1, \rho}\right)-F\left(u_{2, \rho}\right)\right) \\
& \quad-2 \delta \int_{\mathbb{R}^{N}}\left(G\left(v_{1, \rho}+v_{2, \rho}\right)-G\left(v_{1, \rho}\right)-G\left(v_{2, \rho}\right)\right),
\end{aligned}
$$

provided $\rho$ is taken to be sufficiently large.

Step 3. Next we look at $\widetilde{\nu}(\rho)^{2}$. It can be checked that

$$
\begin{aligned}
I^{\prime}\left(u_{1, \rho}+u_{2, \rho}-u, v_{1, \rho}\right. & \left.+v_{2, \rho}-v\right)(\varphi, 0) \\
= & I^{\prime}\left(u_{1, \rho}+u_{2, \rho}, v_{1, \rho}+v_{2, \rho}\right)(\varphi, 0) \\
& \quad-\int_{\mathbb{R}^{N}}\left(f\left(u_{1, \rho}+u_{2, \rho}-u\right)-f\left(u_{1, \rho}+u_{2, \rho}\right)+f(u)\right) \varphi .
\end{aligned}
$$

The term $I^{\prime}\left(u_{1, \rho}+u_{2, \rho}, v_{1, \rho}+v_{2, \rho}\right)(\varphi, 0)$ was estimated in the proof of Lemma 5.5. On the other hand, by applying the Cauchy-Schwarz inequality and the second conclusion in Lemma 5.2 we see that the integral term above is dominated by $\left(\int_{\mathbb{R}^{N}}\left(f\left(u_{1, \rho}+u_{2, \rho}\right) u+f(u)\left(u_{1, \rho}+u_{2, \rho}\right)\right)\right)^{1 / 2}$. As before, it then follows from Lemma 5.7 that

$$
\begin{aligned}
\widetilde{\nu}(\rho)^{2} \leq \delta \int_{\mathbb{R}^{N}}\left(F\left(u_{1, \rho}+u_{2, \rho}\right)-F\right. & \left.\left(u_{1, \rho}\right)-F\left(u_{2, \rho}\right)\right) \\
& \quad+\delta \int_{\mathbb{R}^{N}}\left(G\left(v_{1, \rho}+v_{2, \rho}\right)-G\left(v_{1, \rho}\right)-G\left(v_{2, \rho}\right)\right),
\end{aligned}
$$


provided $\rho$ is taken to be sufficiently large. In conclusion,

$$
I\left(u_{1, \rho}+u_{2, \rho}-u, v_{1, \rho}+v_{2, \rho}-v\right)+C \widetilde{\nu}(\rho)^{2}<3 I(u, v),
$$

and this finishes the proof of Lemma 5.8 .

Lemma 5.9. Assume $\left|y_{1}-y_{2}\right|<1$. There exist $\rho_{0}, R_{0}>0$ such that, for every $R \leq R_{0}$ and $\rho \geq \rho_{0}$,

$$
\begin{array}{r}
\sup \left\{I\left(s\left(u_{1, \rho}+u_{2, \rho}\right) \xi_{R}-t u \xi_{R}+\psi, s\left(v_{1, \rho}+v_{2, \rho}\right) \xi_{R}-t v \xi_{R}-\psi\right): t, s \geq 0, \psi \in H_{0}^{1}(\Omega)\right\} \\
<3 I(u, v) .
\end{array}
$$

Proof. For a fixed $\rho$, the supremum is attained at some $\left(s_{R}, t_{R}, \psi_{R}\right)$. Moreover, $\left(s_{R}+t_{R}\right)_{R}$ and $\left(\left\|\psi_{R}\right\|_{H^{1}\left(\mathbb{R}^{N}\right)}\right)_{R}$ are bounded uniformly in $R$; this can be deduced by arguing as in the first step of the proof included in the Appendix (subsection A.2), by observing that, since $N>2, u \xi_{R} \rightarrow u$ in $H^{1}\left(\mathbb{R}^{N}\right)$ as $R \rightarrow 0$, for every $u \in H^{1}\left(\mathbb{R}^{N}\right)$. Then, up to subsequences, $s_{R} \rightarrow s_{0} \geq 0, t_{R} \rightarrow t_{0} \geq 0, \psi_{R} \rightarrow \psi_{0}$ weakly in $H^{1}\left(\mathbb{R}^{N}\right)$, and

$$
\begin{aligned}
\limsup _{R \rightarrow 0} I\left(s _ { R } \left(u_{1, \rho}+\right.\right. & \left.\left.u_{2, \rho}\right) \xi_{R}-t_{R} u \xi_{R}+\psi_{R}, s_{R}\left(v_{1, \rho}+v_{2, \rho}\right) \xi_{R}-t_{R} v \xi_{R}-\psi_{R}\right) \\
& \leq I\left(s_{0}\left(u_{1, \rho}+u_{2, \rho}\right)-t_{0} u+\psi_{0}, s_{0}\left(v_{1, \rho}+v_{2, \rho}\right)-t_{0} v-\psi_{0}\right) .
\end{aligned}
$$

The conclusion follows from Lemma 5.8

5.2. Proof of Theorems $\mathbf{1 . 1 0}$ and 1.11, Let us consider the system (1.4) with $p, q>1$ satisfying (H1). Thanks to Lemma 4.3 we aim at finding solutions of a truncated system (5.1), where the functions $f$ and $g$ satisfy (fg1)-(fg6) and (fg2').

Following the procedure in [5, 30] we will use the reduced $C^{2}$ functional $\widehat{I}$ : $H_{0}^{1}(\Omega) \rightarrow \mathbb{R}$ defined by

$$
\widehat{I}(\alpha)=I\left(\alpha+\psi_{\alpha}, \alpha-\psi_{\alpha}\right):=\max _{\psi \in H_{0}^{1}(\Omega)} I(\alpha+\psi, \alpha-\psi) .
$$

Arguing as in [5, 30, it can be shown that $\alpha$ is a critical point of $\widehat{I}$ iff $\left(\alpha+\psi_{\alpha}, \alpha-\psi_{\alpha}\right)$ is a critical point of $I$. Similarly to the proof in Lemma 5.4 it can be checked that $\widehat{I}(\alpha) \rightarrow-\infty$ as $\|\alpha\| \rightarrow \infty$, provided $\alpha$ lies in a finite-dimensional subspace of $H_{0}^{1}(\Omega)$.

From now on we fix a minimal $G$-orbit $G x=\left\{y_{1}, \ldots, y_{\ell}\right\}$ in $\mathbb{R}^{N} \backslash\{0\}$ with $\ell>1$. Without loss of generality we can assume that $\left|y_{i}\right|=1$ for every $i$. In case $\ell=2$, we also assume that $\left|y_{1}-y_{2}\right|<2$ (see the statement in Theorem 1.10).

Let

$$
c_{1}^{G}(\Omega):=\inf _{\alpha \in H_{0}^{1}(\Omega)^{G}, \alpha \neq 0} \sup \{\widehat{I}(t \alpha): t \geq 0\} .
$$

As in the previous subsection, we fix a positive, radially symmetric $C^{2}\left(\mathbb{R}^{N}\right) \cap$ $H^{1}\left(\mathbb{R}^{N}\right)$ ground state solution $(u, v)$ of (4.1). Following [7, let

$$
\sigma_{\rho}^{1}(x)=\sum_{i=1}^{\ell} u\left(x-\rho y_{i}\right) \text { and } \sigma_{\rho}^{2}(x)=\sum_{i=1}^{\ell} v\left(x-\rho y_{i}\right) .
$$

Clearly, $\sigma_{\rho}^{1}, \sigma_{\rho}^{2} \in H^{1}\left(\mathbb{R}^{N}\right)^{G}$. Also, let $\xi_{R} \in C^{\infty}\left(\mathbb{R}^{N} ; \mathbb{R}\right)$ be radially symmetric, $\xi_{R}=$ 0 in $B_{R}(0)$, and $\xi_{R}=1$ in $\mathbb{R}^{N} \backslash B_{2 R}(0)$, where $R>0$ is such that $\mathbb{R}^{N} \backslash \Omega \subset B_{R}(0)$. Our next proposition summarizes the content of Lemma 5.6. 
Proposition 5.10. Under the assumptions of Theorem 1.10, the inequality

$$
c_{1}(\Omega)^{G}<\ell c_{0}\left(\mathbb{R}^{N}\right)
$$

holds provided $\rho$ is large enough.

In fact, for simplicity of notation in the quoted lemma we have merely considered the case $\ell=2$. However, it would be a simple task to extend the conclusion to the general case $\ell>1$.

Proof of Theorem 1.10. We denote by $\widehat{N}$ the Nehari manifold associated to $\widehat{I}$ or rather, associated to its restriction to $H_{0}^{1}(\Omega)^{G}$; namely,

$$
\widehat{N}=\left\{\alpha \in H_{0}^{1}(\Omega)^{G}: \alpha \neq 0 \text { and } \widehat{I}^{\prime}(\alpha) \alpha=0\right\} .
$$

It follows from (4.4) that $c_{1}(\Omega)^{G}=\inf _{\widehat{N}} \widehat{I}$. Moreover, it can be shown as in 30, Lemma 2.3] that for every $\alpha \in \widehat{N}, \widehat{I}^{\prime \prime}(\alpha)(\alpha, \alpha)<0$, and so $\widehat{N}$ is a natural constraint for the functional $\widehat{I}$. By using the Ekeland variational principle we can find a Palais-Smale sequence $\left(\alpha_{n}\right)_{n} \subset H_{0}^{1}(\Omega)^{G}, \widehat{I}\left(\alpha_{n}\right) \rightarrow c_{1}(\Omega)^{G}$ and $\widehat{I}^{\prime}\left(\alpha_{n}\right) \rightarrow 0$. The corresponding sequence $\left(u_{n}, v_{n}\right)$ with $u_{n}=\alpha_{n}+\psi_{\alpha_{n}}, v_{n}=\alpha_{n}-\psi_{\alpha_{n}}$ is such that $I\left(u_{n}, v_{n}\right) \rightarrow c_{1}(\Omega)^{G}$ and $I^{\prime}\left(u_{n}, v_{n}\right) \rightarrow 0$. Thanks to Propositions 4.15 ( $(a)$ and 5.10 . up to a subsequence we have strong convergence $\alpha_{n} \rightarrow \alpha_{0}$ in $H_{0}^{1}(\Omega)$, for some $\alpha_{0} \in H_{0}^{1}(\Omega)^{G}$. We have shown that

$$
c_{1}(\Omega)^{G}=\min \left\{I(u, v): u, v \in H_{0}^{1}(\Omega)^{G},(u, v) \neq 0, I^{\prime}(u, v)=0\right\}:=c_{0}(\Omega)^{G} .
$$

Once we know that $c_{0}(\Omega)^{G}$ is attained, we can repeat mutatis mutandis the arguments presented in section 1 , by working now, of course, with the corresponding symmetric Sobolev spaces, so as to conclude that any pair $(u, v) \in H_{0}^{1}(\Omega)^{G} \times$ $H_{0}^{1}(\Omega)^{G}$ such that $I^{\prime}(u, v)=0$ and $I(u, v)=c_{0}(\Omega)^{G}$ satisfies $u v>0$ in $\Omega$. We can then finish as in the final paragraph of the proof of Theorem 4.1.

We now come to the proof of Theorem 1.11, We borrow from [7, Theorem 8] the underlying variational principle. However, since we deal with systems and moreover, due to our truncation technique, we do not work with the pure power case, less explicit computations are available in our setting; also, contrary to the single equation case, the "positive" Nehari manifold $\left\{\alpha: \widehat{I}^{\prime}(\alpha) \alpha^{+}=0\right\}$ will be of no use to us, since in general $\widehat{I}^{\prime}(\alpha) \alpha^{+} \neq \widehat{I}^{\prime}\left(\alpha^{+}\right) \alpha^{+}$. We first restate our previous Lemma 5.9

Proposition 5.11. Under the assumptions of Theorem 1.11, there exists a small $R_{0}>0$ such that if $\mathbb{R}^{N} \backslash \Omega \subset B_{R_{0}}(0)$, then it is possible to find $\rho, R$ in such a way that

$$
\sup \left\{\widehat{I}\left(s \alpha_{0}-t \beta_{0}\right): s, t \geq 0\right\}<(\ell+1) c_{0}\left(\mathbb{R}^{N}\right),
$$

where $\alpha_{0}=\xi_{R}\left(\sigma_{\rho}^{1}+\sigma_{\rho}^{2}\right) / 2$ and $\beta_{0}=\xi_{R}(u+v) / 2$.

Proof of Theorem 1.11. Let

$$
c:=\sup \left\{\widehat{I}\left(s \alpha_{0}-t \beta_{0}\right): s, t \geq 0\right\} .
$$

We assume that $\widehat{I}$ has no sign changing critical points $\alpha$ such that $\widehat{I}(\alpha) \leq c$ and we reach a contradiction in three steps. We point out that, arguing as in Lemma 4.3. see also Remark 4.4, this gives rise to a strong (sign changing) solution of our original system, by taking increasing large truncations of the non-linear terms, 
since the critical levels are bounded from above by a fixed constant, namely by $(\ell+1) c_{0}\left(\mathbb{R}^{N}\right)$

Step 1. For a given small $\varepsilon>0$, let

$$
\mathcal{P}_{\varepsilon}:=\left\{\alpha \in H_{0}^{1}(\Omega)^{G}: \operatorname{dist}(\alpha, P \cup(-P)) \leq \varepsilon\right\},
$$

where $P:=\left\{\alpha \in H_{0}^{1}(\Omega)^{G}: \alpha \geq 0\right\}$. For a given $a \in \mathbb{R}$, we set $\widehat{I}^{a}:=\{\alpha \in$ $\left.H_{0}^{1}(\Omega)^{G}: \widehat{I}(\alpha) \leq a\right\}$. Arguing as in [7, Lemma 11], by using the gradient flow $\eta^{\prime}(t)=-\nabla \widehat{I}(\eta(t))$ and the compactness property implied by Proposition 4.15 $(b)$, we can find a retraction map, $r: \widehat{I}^{c} \rightarrow \widehat{I}^{0} \cup \mathcal{P}_{\varepsilon}$. This means that $r$ is continuous and $r(\alpha)=\alpha$ for every $\alpha \in \widehat{I}^{0} \cup \mathcal{P}_{\varepsilon}$. We mention that in order to construct such a retraction map one must prove that $\mathcal{P}_{\varepsilon}$ is positively invariant with respect to the flow $\eta(t)$; this in turn was settled in [31, Section 4, Lemma 16].

Step 2. We introduce the auxiliary manifold

$$
N_{0}:=\left\{\alpha \in H_{0}^{1}(\Omega)^{G}: I^{\prime}(\alpha, \alpha)(\alpha, \alpha)=0, \alpha \neq 0\right\},
$$

associated to the elliptic equation $-2 \Delta \alpha+2 \alpha=f(\alpha)+g(\alpha)$ in $H_{0}^{1}(\Omega)^{G}$. It is well known that under our assumptions (fg1), (fg2 $\left.2^{\prime}\right)$ and (fg3), we have

$$
\inf _{\alpha \in N_{0}} I(\alpha, \alpha)>0 \quad \text { and } \quad \inf \left\{\|\alpha\|_{L^{r+1}(\Omega)}: \alpha \in N_{0}\right\}>0 .
$$

Also, for every $\alpha \in H_{0}^{1}(\Omega)^{G}, \alpha \neq 0$, there exists a unique positive number $\lambda(\alpha)$ such that $\lambda(\alpha) \alpha \in N_{0}$; since $\inf _{\alpha \in N_{0}} I(\alpha, \alpha)>0$, we have that $\lambda(\alpha) \rightarrow+\infty$ as $\alpha \rightarrow 0$. Finally, it also holds that for every $\alpha \in H_{0}^{1}(\Omega)^{G}$,

$$
\lambda(\alpha)^{r-1} \int_{\mathbb{R}^{N}}|\alpha|^{r+1} \leq C\|\alpha\|_{H_{0}^{1}(\Omega)}^{2} .
$$

In particular, if $\alpha$ lies in a finite-dimensional subspace of $H_{0}^{1}(\Omega)^{G}$ and $\|\alpha\|_{H_{0}^{1}(\Omega)} \rightarrow$ $\infty$, then $\min \left\{\lambda\left(\alpha^{+}\right), \lambda\left(\alpha^{-}\right)\right\} \rightarrow 0$. Without loss of generality, we may assume that $\alpha_{0}, \beta_{0} \in N_{0}$.

Step 3. Our final argument follows the one in [7, Lemma 12]. Let $Q:=\{(s, t) \in$ $\left.\mathbb{R}^{2}: s, t \geq 0\right\}$ and $T:=\{(s, t) \in Q: s+t \leq 1\}$. We introduce the continuous map $\sigma: Q \rightarrow Q$,

$$
\sigma(t, s)=\left(\frac{1}{\lambda\left(r\left(s \alpha_{0}-t \beta_{0}\right)^{+}\right)}, \frac{1}{\lambda\left(r\left(s \alpha_{0}-t \beta_{0}\right)^{-}\right)}\right) .
$$

Clearly, $\sigma(s, t)=(s, t)$ if either $s=0$ or $t=0$. Moreover, as $s+t \rightarrow+\infty$, we have that $\widehat{I}\left(s \alpha_{0}-t \beta_{0}\right)<0$ since $-\widehat{I}$ is coercive over finite-dimensional subspaces of $H_{0}^{1}(\Omega)^{G}$, and so $r\left(s \alpha_{0}-t \beta_{0}\right)=s \alpha_{0}-t \beta_{0}$. It then follows that

$$
\min \left\{\lambda\left(r\left(s \alpha_{0}-t \beta_{0}\right)^{+}\right), \lambda\left(r\left(s \alpha_{0}-t \beta_{0}\right)^{-}\right)\right\} \rightarrow 0,
$$

and therefore

$$
\frac{1}{\lambda\left(r\left(s \alpha_{0}-t \beta_{0}\right)^{+}\right)}+\frac{1}{\lambda\left(r\left(s \alpha_{0}-t \beta_{0}\right)^{+}\right)} \rightarrow+\infty .
$$

As a consequence, we can fix $d_{0}$ sufficiently large so that if $s+t=d_{0}$, then

$$
\frac{1}{\lambda\left(r\left(s \alpha_{0}-t \beta_{0}\right)^{+}\right)}+\frac{1}{\lambda\left(r\left(s \alpha_{0}-t \beta_{0}\right)^{-}\right)}>2 .
$$

Finally, we let $\widetilde{\sigma}: T \rightarrow T$ be given by

$$
\widetilde{\sigma}(s, t)=p\left(\sigma\left(\frac{d_{0}}{2}(s, t)\right)\right)
$$


where $p$ is the natural projection of $Q$ onto $T$ (namely, $p(s, t)=(s, t)$ if $s+t \leq 2$ and $p(s, t)=\frac{2}{s+t}(s, t)$ if $\left.s+t \geq 2\right)$. We see that $\widetilde{\sigma}$ is the identity in the vertices of $T$ and that it maps each side of $T$ into itself. As a consequence, $\widetilde{\sigma}$ is onto; in particular, it assumes the value $(1,1)$, and so we can find $(s, t) \in Q$ such that $\sigma(s, t)=(1,1)$. Going through the definitions, this means that $v:=r(s \alpha-t \beta)$ is such that $v^{ \pm} \neq 0$ and $I^{\prime}\left(v^{ \pm}, v^{ \pm}\right)\left(v^{ \pm}, v^{ \pm}\right)=0$. As pointed out in Step 2 above, we must have that $\operatorname{dist}(v, \mathcal{P})>\varepsilon$ provided $\varepsilon$ was chosen sufficiently small. Since $v \in \widehat{I}^{0} \cup \mathcal{P}_{\varepsilon}$, we deduce that $\widehat{I}(v) \leq 0$. However, since $I\left(v^{ \pm}, v^{ \pm}\right)>0$ and by the very definition of the functional $\widehat{I}$, we have

$$
\widehat{I}(v) \geq I(v, v)=I\left(v^{+}, v^{+}\right)+I\left(v^{-}, v^{-}\right)>0 .
$$

This contradiction completes the proof of Theorem 1.11.

\section{Appendix A. Regularity and Further estimates}

A.1. Proof of Theorem 1.7. This subsection is devoted to the proof of the following two regularity results, from which Theorem 1.7 follows.

Theorem A.1. Assume (H1) and $p q>1$. Let $u \in W^{2, \frac{p+1}{p}}\left(\mathbb{R}^{N}\right)$ be a weak solution of (1.5) and let $v:=|L u|^{\frac{1}{p}-1}$ Lu. Then $u \in W^{2, s}\left(\mathbb{R}^{N}\right)$ and $v \in W^{2, t}\left(\mathbb{R}^{N}\right)$ for all $s$ and $t$ in the range: $\max \left\{1, p^{-1}\right\}<s<\infty, \max \left\{1, q^{-1}\right\}<t<\infty$.

Corollary A.2. Assume $(\mathrm{H} 1)$ and $p q>1$. Let $u \in W^{2, \frac{p+1}{p}}\left(\mathbb{R}^{N}\right)$ be a weak solution of (1.5) and let $v:=|L u|^{\frac{1}{p}-1} L u$. Then $u \in C^{2, \alpha}\left(\mathbb{R}^{N}\right)$ and $v \in C^{2, \beta}\left(\mathbb{R}^{N}\right)$ for all $\alpha$ and $\beta$ in the range: $0<\alpha \leq \min \{1, p\}$ and $0<\beta \leq \min \{1, q\}$, and $(u, v)$ is a classical solution for (1.4).

Proof of Theorem A.1. The argument follows the lines of [19, Theorem 1.1] in the bounded domain case. We split the proof into two cases, according to whether $p(N-2)-2 \leq 0$ or $p(N-2)-2>0$.

Case 1. $p(N-2)-2 \leq 0$. The condition $p(N-2)-2 \leq 0$ guarantees, by means of some Sobolev embedding, that $W^{2, \frac{p+1}{p}}\left(\mathbb{R}^{N}\right) \hookrightarrow L^{r}\left(\mathbb{R}^{N}\right)$ for all $\frac{p+1}{p} \leq r<\infty$. On the other hand, [21, Theorem 9.15] insures the existence of a unique strong solution of $L w=|u|^{q-1} u$ in $\mathbb{R}^{N}$ and $w \in W^{2, t}\left(\mathbb{R}^{N}\right)$ for all

$$
\infty>t \begin{cases}\geq \frac{p+1}{p} \frac{1}{q} & \text { if } \quad \frac{p+1}{p} \frac{1}{q}>1 \\ >1 & \text { if } \quad \frac{p+1}{p} \frac{1}{q} \leq 1 .\end{cases}
$$

We note that in case $\frac{p+1}{p} \frac{1}{q} \leq 1$ we have that $|u|^{q-1} u \in L^{t}\left(\mathbb{R}^{N}\right)$ for all $1 \leq t<\infty$. Now, again from [21, Theorem 9.15], there exists a unique strong solution of $L z=$ $|w|^{p-1} w$ in $R^{N}$ and $z \in W^{2, s}\left(\mathbb{R}^{N}\right)$ for all

$$
\infty>s \begin{cases}\geq \frac{p+1}{p} \frac{1}{p q} & \text { if } \quad \frac{p+1}{p} \frac{1}{q}>1 \text { and } \frac{p+1}{p} \frac{1}{p q}>1 \\ >\max \left\{1, p^{-1}\right\} & \text { if } \quad \frac{p+1}{p q} \leq 1 \text { or } \frac{p+1}{p} \frac{1}{p q} \leq 1 .\end{cases}
$$


Since $p q>1$, it follows that $z \in W^{2, \frac{p+1}{p}}\left(\mathbb{R}^{N}\right)$. Given $\psi \in \mathcal{D}\left(\mathbb{R}^{N}\right)$, let $\varphi \in$ $W^{2, \frac{p+1}{p}}\left(\mathbb{R}^{N}\right)$ be the strong solution of $L \varphi=\psi$ in $\mathbb{R}^{N}$. So, for all $\psi=L \varphi \in \mathcal{D}\left(\mathbb{R}^{N}\right)$,

$$
\begin{aligned}
\int_{\mathbb{R}^{N}}|L z|^{\frac{1}{p}-1} L z L \varphi d x=\int_{\mathbb{R}^{N}} w L \varphi d x & =\int_{\mathbb{R}^{N}}(L w) \varphi d x \\
& =\int_{\mathbb{R}^{N}}|u|^{q-1} u \varphi d x=\int_{\mathbb{R}^{N}}|L u|^{\frac{1}{p}-1} L u L \varphi d x .
\end{aligned}
$$

It follows that $z=u$ and $w=|L z|^{\frac{1}{p}-1} L z=|L u|^{\frac{1}{p}-1} L u=v$. Thus $(u, v)$ is a strong solution of (1.4) and

$$
u \in W^{2, s}\left(\mathbb{R}^{N}\right) \text { for all } \infty>s \begin{cases}\geq \frac{p+1}{p} \frac{1}{p q} & \text { if } \quad \frac{p+1}{p} \frac{1}{q}>1 \text { and } \frac{p+1}{p} \frac{1}{p q}>1 \\ >\max \left\{1, p^{-1}\right\} & \text { if } \quad \frac{p+1}{p} \frac{1}{q} \leq 1 \text { or } \frac{p+1}{p} \frac{1}{p q} \leq 1 .\end{cases}
$$

Let $n=\min \left\{m \in \mathbb{N}: \frac{p+1}{p} \frac{1}{q} \frac{1}{(p q)^{m-1}} \leq 1\right.$ or $\left.\frac{p+1}{p} \frac{1}{(p q)^{m}} \leq 1\right\}$. By applying $n$ consecutive times Lemma A.3 hereafter to the system (1.4), based on the fact that $p q>1$, it follows that $u \in W^{2, s}\left(\mathbb{R}^{N}\right), v \in W^{2, t}\left(\mathbb{R}^{N}\right)$ for all $\max \left\{1, p^{-1}\right\}<s<\infty$, $\max \left\{1, q^{-1}\right\}<t<\infty$.

Case 2. $p(N-2)-2>0$. Without loss of generality, one can also assume that $q(N-2)-2>0$. Otherwise, one can proceed similarly as in Case 1 . In particular we can fix $\bar{q}>q$ and $\bar{p}>p$ in such a way that

$$
\frac{1}{p+1}+\frac{1}{\bar{q}+1}=1-\frac{2}{N}, \frac{1}{\bar{p}+1}+\frac{1}{q+1}=1-\frac{2}{N} \text {. }
$$

We observe that $W^{2, \frac{p+1}{p}}\left(\mathbb{R}^{N}\right) \hookrightarrow L^{\bar{q}+1}\left(\mathbb{R}^{N}\right)$. Let $w$ be defined as in Case 1 . Then

$$
w \in W^{2, t}\left(\mathbb{R}^{N}\right) \text { for all } \frac{\bar{q}+1}{q} \geq t \begin{cases}\geq \frac{p+1}{p} \frac{1}{q} & \text { if } \quad \frac{p+1}{p} \frac{1}{q}>1 \\ >1 & \text { if } \quad \frac{p+1}{p} \frac{1}{q} \leq 1\end{cases}
$$

Let $z$ be defined as in Case 1. By using the Sobolev embedding of $W^{2, \frac{\bar{q}+1}{q}}\left(\mathbb{R}^{N}\right)$, it follows that $z \in W^{2, s}\left(\mathbb{R}^{N}\right)$ for all values of $s$ in the following ranges:

$$
\left\{\begin{array}{l}
s \geq \frac{p+1}{p} \frac{1}{p q}, \text { if } \frac{p+1}{p} \frac{1}{q}>1 \text { and } \frac{p+1}{p} \frac{1}{p q}>1 \\
s>\max \left\{1, p^{-1}\right\}, \text { if } \frac{p+1}{p q} \leq 1 \text { or } \frac{p+1}{p} \frac{1}{p q} \leq 1 \\
\text { and } \\
s \leq \frac{\tilde{q}+1}{p}:=\frac{(\bar{q}+1) N}{q N-2(\bar{q}+1)} \frac{1}{p}, \text { if } q N-2(\bar{q}+1)>0 \\
s<\infty, \quad \text { if } q N-2(\bar{q}+1) \leq 0 .
\end{array}\right.
$$

As for the fraction $\frac{\tilde{q}+1}{p}$ corresponding to the case when $q N-2(\bar{q}+1)>0$, we have that $\frac{\tilde{q}+1}{p}>\frac{p+1}{p}$. Indeed,

$$
\begin{aligned}
\frac{\tilde{q}+1}{p+1}=\frac{(\bar{q}+1) N}{q N-2(\bar{q}+1)} \frac{1}{p+1} & =\frac{\bar{q}+1}{p+1} \frac{N}{q(N-2)-2(\bar{q}-q)-2} \\
& >\frac{\bar{q}+1}{p+1} \frac{N}{q(N-2)-2}=\frac{\bar{q}+1}{p+1} \frac{\bar{p}+1}{q+1}=: \gamma>1 .
\end{aligned}
$$

We conclude that $z \in W^{2, \frac{p+1}{p}}\left(\mathbb{R}^{N}\right)$, and it follows as in Case 1 that $z=u$. Thus, by setting $v:=|L u|^{\frac{1}{p}-1} L u$, it follows that $(u, v)$ is a strong solution of (1.4). We 
iterate this construction by applying Lemmas A.3 and A.4 hereafter yielding that $u \in W^{2, s}\left(\mathbb{R}^{N}\right), v \in W^{2, t}\left(\mathbb{R}^{N}\right)$ for all $\max \left\{1, p^{-1}\right\}<s<\infty, \max \left\{1, q^{-1}\right\}<t<$ $\infty$.

Lemma A.3. Assume (H1) and $p q>1$, and let $(u, v)$ be a strong solution of (1.4). Assume that, for some $n \in \mathbb{N}, u \in W^{2, s}\left(\mathbb{R}^{N}\right)$ for all

$$
\frac{p+1}{p} \geq s \begin{cases}\geq \frac{p+1}{p} \frac{1}{(p q)^{n}} & \text { if } \quad \frac{p+1}{p} \frac{1}{q} \frac{1}{(p q)^{n-1}}>1 \text { and } \frac{p+1}{p} \frac{1}{(p q)^{n}}>1 \\ >\max \left\{1, p^{-1}\right\} & \text { if } \quad \frac{p+1}{p} \frac{1}{q} \frac{1}{(p q)^{n-1}} \leq 1 \text { or } \frac{p+1}{p} \frac{1}{(p q)^{n}} \leq 1 .\end{cases}
$$

Then $v \in W^{2, t}\left(\mathbb{R}^{N}\right)$ for all

$$
\frac{q+1}{q} \geq t \begin{cases}\geq \frac{p+1}{p} \frac{1}{q} \frac{1}{(p q)^{n}} & \text { if } \quad \frac{p+1}{p} \frac{1}{q} \frac{1}{(p q)^{n}}>1 \text { and } \frac{p+1}{p} \frac{1}{(p q)^{n}}>1, \\ >\max \left\{1, q^{-1}\right\} & \text { if } \quad \frac{p+1}{p} \frac{1}{q} \frac{1}{(p q)^{n}} \leq 1 \text { or } \frac{p+1}{p} \frac{1}{(p q)^{n}} \leq 1\end{cases}
$$

and $u \in W^{2, s}\left(\mathbb{R}^{N}\right)$ for all

$$
\frac{p+1}{p} \geq s \begin{cases}\geq \frac{p+1}{p} \frac{1}{(p q)^{n+1}} & \text { if } \quad \frac{p+1}{p} \frac{1}{q} \frac{1}{(p q)^{n}}>1 \text { and } \frac{p+1}{p} \frac{1}{(p q)^{n+1}}>1, \\ >\max \left\{1, p^{-1}\right\} & \text { if } \quad \frac{p+1}{p} \frac{1}{q} \frac{1}{(p q)^{n}} \leq 1 \text { or } \frac{p+1}{p} \frac{1}{(p q)^{n+1}} \leq 1 .\end{cases}
$$

In addition, if one replaces in (A.4), on the left-hand side of $s, \frac{p+1}{p}$ by $\infty$ (with a strict inequality), then one can replace in (A.5), on the left-hand side of $t, \frac{q+1}{q}$ by $\infty$ (with a strict inequality).

Proof. Since $E=W^{2, \frac{p+1}{p}}\left(\mathbb{R}^{N}\right) \hookrightarrow L^{q+1}\left(\mathbb{R}^{N}\right)$, it follows from (1.4) and 21, Theorem 9.15] that $v \in W^{2, t}\left(\mathbb{R}^{N}\right)$ for all

$$
\frac{q+1}{q} \geq t \begin{cases}\geq \frac{p+1}{p} \frac{1}{q} \frac{1}{(p q)^{n}} & \text { if } \quad \frac{p+1}{p} \frac{1}{q} \frac{1}{(p q)^{n}}>1 \text { and } \frac{p+1}{p} \frac{1}{(p q)^{n}}>1 \\ >\max \left\{1, q^{-1}\right\} & \text { if } \quad \frac{p+1}{p} \frac{1}{q} \frac{1}{(p q)^{n}} \leq 1 \text { or } \frac{p+1}{p} \frac{1}{(p q)^{n}} \leq 1 .\end{cases}
$$

Now, since $W^{2, \frac{q+1}{q}}\left(\mathbb{R}^{N}\right) \hookrightarrow L^{p+1}\left(\mathbb{R}^{N}\right)$ it follows from (1.4) and 21, Theorem 9.15] that $u \in W^{2, s}\left(\mathbb{R}^{N}\right)$ for all

$$
\frac{p+1}{p} \geq s \begin{cases}\geq \frac{p+1}{p} \frac{1}{(p q)^{n+1}} & \text { if } \quad \frac{p+1}{p} \frac{1}{q} \frac{1}{(p q)^{n}}>1 \text { and } \frac{p+1}{p} \frac{1}{(p q)^{n+1}}>1 \\ >\max \left\{1, p^{-1}\right\} & \text { if } \quad \frac{p+1}{p} \frac{1}{q} \frac{1}{(p q)^{n}} \leq 1 \text { or } \frac{p+1}{p} \frac{1}{(p q)^{n+1}} \leq 1 .\end{cases}
$$

Lemma A.4. Assume (H1) and $p q>1$, and let $(u, v)$ be a strong solution of (1.4). Suppose that $u \in W^{2, s}\left(\mathbb{R}^{N}\right)$ for all $\frac{p+1}{p} \leq s \leq \frac{r+1}{r}$, for some $0<r \leq p$. In the case when $r(N-2)-2>0$, let $\bar{s}>r$ be such that $\frac{1}{r+1}+\frac{1}{\bar{s}+1}=1-\frac{2}{N}$. Then $v \in W^{2, t}\left(\mathbb{R}^{N}\right)$ for all values of $t$ in the range:

$$
\begin{aligned}
& \text { if } \left.\frac{p+1}{p} \frac{1}{q}>1 \text {, then } \frac{p+1}{p} \frac{1}{q} \leq\right\} t\left\{\leq \frac{\bar{s}+1}{q} \text { if } r(N-2)-2>0\right. \text {, } \\
& \text { if } \left.\frac{p+1}{p} \frac{1}{q} \leq 1 \text {, then } \max \left\{1, q^{-1}\right\}<\right\}<t\left\{\begin{aligned}
& < \\
<\infty & \text { if } r(N-2)-2 \leq 0
\end{aligned}\right.
\end{aligned}
$$

and $u \in W^{2, s}\left(\mathbb{R}^{N}\right)$ for all values of $s$ in the range:

$$
\left\{\begin{array}{l}
s \geq \frac{p+1}{p} \frac{1}{p q}, \text { if } \frac{p+1}{p} \frac{1}{q}>1 \text { and } \frac{p+1}{p} \frac{1}{p q}>1 \\
s>\max \left\{1, p^{-1}\right\}, \text { if } \frac{p+1}{p} \frac{1}{q} \leq 1 \text { or } \frac{p+1}{p} \frac{1}{p q} \leq 1 \\
\text { and } \\
s \leq \frac{(\bar{s}+1) N}{(q N-2(\bar{s}+1))} \frac{1}{p}, \text { if } r(N-2)-2>0 \text { and } q N-2(\bar{s}+1)>0 \\
s<\infty, \text { if } r(N-2)-2 \leq 0 \text { or } q N-2(\bar{s}+1) \leq 0
\end{array}\right.
$$


In addition, in the case when $r(N-2)-2>0$ and $q N-2(\bar{s}+1)>0$, the ratio between $\frac{(\bar{s}+1) N}{(q N-2(\bar{s}+1))} \frac{1}{p}$ and $\frac{r+1}{r}$ is greater than $\gamma:=\frac{\bar{q}+1}{p+1} \frac{\bar{p}+1}{q+1}>1$.

Proof. The left-hand sides of (A.6) and (A.7) follow from (A.1) and (A.2), respectively. If $r(N-2)-2 \leq 0$, then $W^{2, \frac{r+1}{r}}\left(\mathbb{R}^{N}\right) \hookrightarrow L^{t}\left(\mathbb{R}^{N}\right)$ for all $\frac{r+1}{r} \leq t<\infty$ and the right-hand sides of (A.6) and A.7) follow straightforwardly. In the sequel we assume $r(N-2)-2>0$. Then there exists $\bar{s}>r$ such that

$$
\frac{1}{r+1}+\frac{1}{\bar{s}+1}=1-\frac{2}{N}
$$

Since $r \leq p$, one has $\bar{s} \geq \bar{q}$ and then [21, Theorem 9.15] yields that $v \in W^{2, \frac{\bar{s}+1}{q}}\left(\mathbb{R}^{N}\right)$. Again by [21, Theorem 9.15] we conclude that $u \in W^{2, s}\left(\mathbb{R}^{N}\right)$ as on the right-hand side of (A.7).

As for the final conclusion in Lemma A.4. we have that

$$
\begin{aligned}
& \frac{(\bar{s}+1) N}{(q N-2(\bar{s}+1))} \frac{1}{p} \frac{r}{r+1}=\frac{r}{q(r(N-2)-2)-2(r+1)} \frac{N}{p} \\
& \quad \geq \frac{p}{q(p(N-2)-2)-2(p+1)} \frac{N}{p}=\frac{\bar{q}+1}{p+1} \frac{N}{q(N-2)-2(\bar{q}-q)-2}>\gamma>1,
\end{aligned}
$$

where, besides (A.3), we have used the following facts: $\bar{s}+1=\frac{(r+1) N}{r(N-2)-2}$; the function $f(t):=\frac{t}{q(t(N-2)-2)-2(t+1)}$ is decreasing in the interval $[r, p] ; \frac{\bar{q}+1}{p+1}=\frac{N}{p(N-2)-2}$.

Proof of Corollary A.2. Theorem A.1 combined with some Sobolev embeddings, guarantees that $u, v \in C^{1, \gamma}\left(\mathbb{R}^{N}\right)$ for some $0<\gamma \leq 1$.

Now, we observe that if $r>1$ and $w \in C^{1, \gamma}\left(\mathbb{R}^{N}\right)$, then $|w|^{r-1} w \in C^{1, \alpha}\left(\mathbb{R}^{N}\right)$, with

$$
\alpha=\min \{\gamma, f(r)\}, \quad f(r):=\left\{\begin{array}{l}
1 \text { if } r \in \mathbb{N}, \\
r-[r] \text { if } r \notin \mathbb{N} .
\end{array}\right.
$$

In particular, $|w|^{r-1} w \in C^{0,1}\left(\mathbb{R}^{N}\right)$, while if $0<r \leq 1$ and $w \in C^{0,1}\left(\mathbb{R}^{N}\right)$, then the inequality

$$
\left.|| a\right|^{r-1} a-|b|^{r-1} b\left|\leq 2^{1-r}\right| a-\left.b\right|^{r} \quad \forall a, b \in \mathbb{R}
$$

yields that $|w|^{r-1} w \in C^{0, r}\left(\mathbb{R}^{N}\right)$.

By combining this remark with the fact that $u, v \in C^{1, \gamma}\left(\mathbb{R}^{N}\right)$, and by using Schauder's estimates, the desired regularity for $u$ and $v$ is obtained.

A.2. Additional estimates. This subsection is devoted to a detailed proof of Step 1 of the proof of Lemma 5.8. We split the argument into several steps.

Step 1. We denote $u_{\rho}=u_{1, \rho}+u_{2, \rho}, v_{\rho}=v_{1, \rho}+v_{2, \rho}$. For a large, fixed $\rho>0$, we can write

$$
I\left(s u_{\rho}-t u+\psi, s v_{\rho}-t v-\psi\right)=I\left((s+t) u_{s, t}+\psi,(s+t) v_{s, t}-\psi\right),
$$

with $u_{s, t}:=\frac{s}{s+t} u_{\rho}-\frac{t}{s+t} u$ and $v_{s, t}:=\frac{s}{s+t} v_{\rho}-\frac{t}{s+t} v$. Since for any fixed $\mu>2$,

$$
\inf _{s, t \geq 0}\left\|u_{s, t}+v_{s, t}\right\|_{L^{\mu}\left(\mathbb{R}^{N}\right)}>0,
$$

it can be shown that the above quantity tends to $-\infty$ as $s+t \rightarrow+\infty$, uniformly in $\psi \in H^{1}\left(\mathbb{R}^{N}\right)$ (cf. the proof of Lemma 5.4). As a consequence, the left-hand side in the statement of Lemma 5.8 is attained at some $\left(s_{\rho}, t_{\rho}, \psi_{\rho}\right)$. In fact, we have 
$\liminf \inf _{\rho \rightarrow \infty} \inf _{s, t \geq 0}\left\|u_{s, t}+v_{s, t}\right\|_{L^{\mu}\left(\mathbb{R}^{N}\right)}>0$, and so $s_{\rho}+t_{\rho}$ is bounded uniformly in $\rho$. By arguing as in the proof of Lemma 5.4, we also have that $\psi_{\rho}$ is bounded in $H^{1}\left(\mathbb{R}^{N}\right) \cap L^{\infty}\left(\mathbb{R}^{N}\right)$ independently of $\rho$ and of the truncation procedure in Lemma 4.3. In particular, in the sequel we can work with a fixed truncated problem, so that the property $\left(\mathrm{fg} 2^{\prime}\right)$ in Proposition 4.2 holds.

Step 2. We claim that $s_{\rho} \rightarrow 1$ and $t_{\rho} \rightarrow 1$ as $\rho \rightarrow \infty$. To prove this, we first collect some basic facts from [30, Section 2]. Since $I^{\prime}(u, v)=0$, we have that

$$
I(u, v)=\sup \left\{I(t u+\psi, t v-\psi): t \geq 0, \psi \in H^{1}\left(\mathbb{R}^{N}\right)\right\} .
$$

We recall the reduced $C^{2}$ functional $\widehat{I}: H^{1}\left(\mathbb{R}^{N}\right) \rightarrow \mathbb{R}$ introduced in subsection 5.2.

$$
\widehat{I}(\alpha):=\max _{\psi \in H^{1}\left(\mathbb{R}^{N}\right)} I(\alpha+\psi, \alpha-\psi)=I\left(\alpha+\psi_{\alpha}, \alpha-\psi_{\alpha}\right) .
$$

Then, by letting $\alpha:=(u+v) / 2$, we have

$$
I(u, v)=\widehat{I}(\alpha)=\sup _{t \geq 0} \widehat{I}(t \alpha)>\widehat{I}\left(t_{0} \alpha\right), \quad \forall t_{0} \geq 0, t_{0} \neq 1 .
$$

Finally, we now show that if $\alpha_{n} \rightarrow \alpha_{0}$ weakly in $H^{1}\left(\mathbb{R}^{N}\right)$, then

$$
\widehat{I}\left(\alpha_{n}-\alpha_{0}\right)=\widehat{I}\left(\alpha_{n}\right)-\widehat{I}\left(\alpha_{0}\right)+\mathrm{o}(1) \quad \text { as } n \rightarrow \infty .
$$

Indeed, let $u_{n}:=\alpha_{n}+\psi_{n}, v_{n}:=\alpha_{n}-\psi_{n}, u_{0}:=\alpha_{0}+\psi_{0}, v_{0}:=\alpha_{0}-\psi_{0}$, with $\psi_{n}=\psi_{\alpha_{n}}$ and $\psi_{0}=\psi_{\alpha_{0}}$. Since $u_{n} \rightarrow u_{0}$ weakly in $H^{1}\left(\mathbb{R}^{N}\right)$ and since $|f(s) s| \leq$ $C\left(s^{2}+|s|^{r+1}\right)$ with $r<(N+2) /(N-2)$, it follows easily that

$$
\int_{\mathbb{R}^{N}} F\left(u_{n}-u_{0}\right)=\int_{\mathbb{R}^{N}} F\left(u_{n}\right)-\int_{\mathbb{R}^{N}} F\left(u_{0}\right)+\mathrm{o}(1) .
$$

Proceeding similarly with $v_{n}$ and by using the definition of $\widehat{I}$, we deduce that

$$
\begin{aligned}
\widehat{I}\left(\alpha_{n}\right)-\widehat{I}\left(\alpha_{0}\right) & :=I\left(\alpha_{n}+\psi_{n}, \alpha_{n}-\psi_{n}\right)-I\left(\alpha_{0}+\psi_{0}, \alpha_{0}-\psi_{0}\right) \\
& =I\left(\alpha_{n}-\alpha_{0}+\psi_{n}-\psi_{0}, \alpha_{n}-\alpha_{0}-\psi_{n}+\psi_{0}\right)+\mathrm{o}(1) \\
& \leq \widehat{I}\left(\alpha_{n}-\alpha_{0}\right)+\mathrm{o}(1) .
\end{aligned}
$$

Concerning the reversed inequality, let us fix $\widetilde{\psi}_{n}$ such that

$$
\widehat{I}\left(\alpha_{n}-\alpha_{0}\right)=I\left(\alpha_{n}-\alpha_{0}+\widetilde{\psi}_{n}, \alpha_{n}-\alpha_{0}-\widetilde{\psi}_{n}\right) .
$$

Then $\widetilde{\psi}_{n} \rightarrow 0$ weakly in $H^{1}\left(\mathbb{R}^{N}\right)$ and, as before,

$$
\begin{aligned}
& \int_{\mathbb{R}^{N}} F\left(\alpha_{n}-\alpha_{0}+\widetilde{\psi}_{n}\right)=\int_{\mathbb{R}^{N}} F\left(\left(\alpha_{n}+\widetilde{\psi}_{n}+\psi_{0}\right)-u_{0}\right) \\
& \quad=\int_{\mathbb{R}^{N}} F\left(\alpha_{n}+\widetilde{\psi}_{n}+\psi_{0}\right)-\int_{\mathbb{R}^{N}} F\left(u_{0}\right)+\mathrm{o}(1) .
\end{aligned}
$$

Again by the definition of the functional $\widehat{I}$,

$$
\begin{aligned}
\widehat{I}\left(\alpha_{n}-\alpha_{0}\right) & -\widehat{I}\left(\alpha_{n}\right)+\widehat{I}\left(\alpha_{0}\right) \\
\leq & \widehat{I}\left(\alpha_{n}-\alpha_{0}\right)-I\left(\alpha_{n}+\widetilde{\psi}_{n}+\psi_{0}, \alpha_{n}-\widetilde{\psi}_{n}-\psi_{0}\right)+\widehat{I}\left(\alpha_{0}\right) \\
& =2\left\langle\left(\alpha_{0}-\alpha_{n}\right), \alpha_{0}\right\rangle+2\left\langle\widetilde{\psi}_{n}, \psi_{0}\right\rangle+\mathrm{o}(1)=\mathrm{o}(1),
\end{aligned}
$$

where we have denoted $\langle u, v\rangle:=\int_{\mathbb{R}^{N}}(\langle\nabla u, \nabla v\rangle+u v)$. 
Step 3. Going back to our proof in Step 1, we may assume that $s_{\rho} \rightarrow s_{0}$ and $t_{\rho} \rightarrow t_{0}$ for some $s_{0}, t_{0} \geq 0$. In order to prove our claim that $t_{0}=s_{0}=1$, it is therefore sufficient to prove that

$$
I\left(s_{\rho} u_{\rho}-t_{\rho} u+\psi_{\rho}, s_{\rho} v_{\rho}-t_{\rho} v-\psi_{\rho}\right)=2 \widehat{I}\left(s_{0} \alpha\right)+\widehat{I}\left(t_{0} \alpha\right)+\mathrm{o}(1)
$$

and

$$
I\left(s_{\rho} u_{\rho}-t_{\rho} u+\psi_{\rho}, s_{\rho} v_{\rho}-t_{\rho} v-\psi_{\rho}\right) \geq 3 \widehat{I}(\alpha)+\mathrm{o}(1)
$$

as $\rho \rightarrow \infty$. We prove the first identity. Let $\alpha_{\rho}:=\left(u_{\rho}+v_{\rho}\right) / 2$. By the definition of $\widehat{I}$

$$
I\left(s_{\rho} u_{\rho}-t_{\rho} u+\psi_{\rho}, s_{\rho} v_{\rho}-t_{\rho} v-\psi_{\rho}\right)=\widehat{I}\left(s_{\rho} \alpha_{\rho}-t_{\rho} \alpha\right)
$$

and, of course,

$$
\widehat{I}\left(s_{\rho} \alpha_{\rho}-t_{\rho} \alpha\right)=\widehat{I}\left(s_{0} \alpha_{\rho}-t_{0} \alpha\right)+\mathrm{o}(1) .
$$

Since $s_{0} \alpha_{\rho}-t_{0} \alpha \rightarrow-t_{0} \alpha$ weakly in $H^{1}\left(\mathbb{R}^{N}\right)$, it was proved in Step 2 above that

$$
\widehat{I}\left(s_{0} \alpha_{\rho}-t_{0} \alpha\right)=\widehat{I}\left(s_{0} \alpha_{\rho}\right)+\widehat{I}\left(-t_{0} \alpha\right)+\mathrm{o}(1)=\widehat{I}\left(s_{0} \alpha_{\rho}\right)+\widehat{I}\left(t_{0} \alpha\right)+\mathrm{o}(1) ;
$$

we observe that in the second equality we have used the fact that $I$ is an even functional (thus so is $\widehat{I}$ ). In conclusion,

$$
I\left(s_{\rho} u_{\rho}-t_{\rho} u+\psi_{\rho}, s_{\rho} v_{\rho}-t_{\rho} v-\psi_{\rho}\right)=\widehat{I}\left(s_{0} \alpha_{\rho}\right)+\widehat{I}\left(t_{0} \alpha\right)+\mathrm{o}(1) .
$$

On the other hand, let

$$
\widetilde{u}_{\rho}(x)=u\left(x-\rho y_{1}+\rho y_{2}\right), \quad \widetilde{v}_{\rho}(x)=v\left(x-\rho y_{1}+\rho y_{2}\right), \quad \widetilde{\alpha}_{\rho}(x)=(\widetilde{u} \rho+\widetilde{v} \rho) / 2 .
$$

By using a change of variables, we see that

$$
\widehat{I}\left(s_{0} \widetilde{\alpha}_{\rho}\right)=\widehat{I}\left(s_{0} \alpha\right) \quad \text { and } \quad \widehat{I}\left(s_{0} \alpha_{\rho}\right)=\widehat{I}\left(s_{0} \widetilde{\alpha}_{\rho}+s_{0} \alpha\right) .
$$

Since $s_{0} \widetilde{\alpha}_{\rho}+s_{0} \alpha \rightarrow s_{0} \alpha$ weakly in $H^{1}\left(\mathbb{R}^{N}\right)$,

$$
\widehat{I}\left(s_{0} \widetilde{\alpha}_{\rho}+s_{0} \alpha\right)=\widehat{I}\left(s_{0} \widetilde{\alpha}_{\rho}\right)+\widehat{I}\left(s_{0} \alpha\right)+\mathrm{o}(1) .
$$

By combining (A.11) and (A.12),

$$
\widehat{I}\left(s_{0} \alpha_{\rho}\right)=\widehat{I}\left(s_{0} \widetilde{\alpha}_{\rho}\right)+\widehat{I}\left(s_{0} \alpha\right)+\mathrm{o}(1)=\widehat{I}\left(s_{0} \alpha\right)+\widehat{I}\left(s_{0} \alpha\right)+\mathrm{o}(1),
$$

and (A.8) follows from (A.10) and (A.13). Since $I\left(s_{\rho} u_{\rho}-t_{\rho} u+\psi_{\rho}, s_{\rho} v_{\rho}-t_{\rho} v-\psi_{\rho}\right) \geq$ $\widehat{I}\left(\alpha_{\rho}-\alpha\right)$ by definition of $s_{\rho}, t_{\rho}$ and $\psi_{\rho}$, in a similar way we deduce A.9.

Step 4. Let

$$
\begin{aligned}
\theta(s, t) & :=\max \left\{I\left(s u_{\rho}-t u+\psi, s v_{\rho}-t v-\psi\right): \psi \in H^{1}\left(\mathbb{R}^{N}\right)\right\} \\
& =I\left(s u_{\rho}-t u+\psi_{s, t}, s v_{\rho}-t v-\psi_{s, t}\right) .
\end{aligned}
$$

We aim at proving that

$$
\theta\left(s_{\rho}, t_{\rho}\right) \leq I\left(u_{\rho}-u, v_{\rho}-v\right)+C \widetilde{\nu}(\rho)^{2},
$$

where

$$
\widetilde{\nu}(\rho)=\sup \left\{I^{\prime}\left(u_{\rho}-u, v_{\rho}-v\right)(\varphi, \psi):\|\varphi\|_{H^{1}\left(\mathbb{R}^{N}\right)}+\|\psi\|_{H^{1}\left(\mathbb{R}^{N}\right)} \leq 1\right\} .
$$

In order to prove our claim it is sufficient to establish the following estimates:

(i) $\quad\left\|\psi_{1,1}\right\|_{H^{1}\left(\mathbb{R}^{N}\right)} \leq C \widetilde{\nu}(\rho)$;

(ii) $\limsup _{\rho \rightarrow \infty} \frac{\partial^{2} \theta}{\partial s^{2}}(1,1)<0$; 
(iii) $\limsup _{\rho \rightarrow \infty} \frac{\partial^{2} \theta}{\partial t^{2}}(1,1)<0$;

(iv) $\lim _{\rho \rightarrow \infty} \frac{\partial^{2} \theta}{\partial s \partial t}(1,1)=\lim _{\rho \rightarrow \infty} \frac{\partial^{2} \theta}{\partial t \partial s}(1,1)=0$.

Indeed, since we already know that $s_{\rho} \rightarrow 1$ and $t_{\rho} \rightarrow 1$, it follows immediately from (i)-(iv) that $\left|s_{\rho}-1\right|+\left|t_{\rho}-1\right| \leq C \widetilde{\nu}(\rho)$. The final conclusion can then be derived similarly to [30, page 8].

Step 5 . The function $\psi=\psi_{1,1}$ is defined by the relation $I^{\prime}\left(u_{\rho}-u+\psi, v_{\rho}-v-\right.$ $\psi)(\varphi,-\varphi)=0 \forall \varphi \in H^{1}\left(\mathbb{R}^{N}\right)$; that is, $\psi$ is the unique solution of the equation in $H^{1}\left(\mathbb{R}^{N}\right)$ :

$$
-2 \Delta \psi+2 \psi=f\left(u_{\rho}\right)-f(u)-f\left(u_{\rho}-u+\psi\right)+g\left(v_{\rho}-v-\psi\right)+g(v)-g\left(v_{\rho}\right) .
$$

We multiply by $\psi$ and integrate by parts. Since $f^{\prime}, g^{\prime} \geq 0$, we deduce that

$$
\begin{aligned}
2\|\psi\|_{H^{1}\left(\mathbb{R}^{N}\right)}^{2} & \leq \int_{\mathbb{R}^{N}}\left(f\left(u_{\rho}\right)-f(u)-f\left(u_{\rho}-u\right)+g\left(v_{\rho}-v\right)+g(v)-g\left(v_{\rho}\right)\right) \psi \\
& =I^{\prime}\left(u_{\rho}-u, v_{\rho}-v\right)(\psi,-\psi),
\end{aligned}
$$

and so $\|\psi\|_{H^{1}\left(\mathbb{R}^{N}\right)} \leq \widetilde{\nu}(\rho)$. This is estimate (i) above.

Step 6 . The estimate in (ii) relies heavily on the following. We denote by $\bar{f}$ the even map $\bar{f}(s)=f(s) / s$; we recall from $(f g 1)$ and $\left(f g 2^{\prime}\right)$ that $|\bar{f}(s)| \leq C\left(1+|s|^{r-1}\right)$ with $r>1$ and $(r-1)(N-2)<(N+2)$; however, in general, $\bar{f}$ is not a $C^{1}$ function. We prove that

$$
\int_{\mathbb{R}^{N}} \bar{f}\left(u_{\rho}-u+\psi\right) u_{\rho} \phi=\int_{\mathbb{R}^{N}} \bar{f}\left(u_{\rho}\right) u_{\rho} \phi+\mathrm{o}(1)
$$

as $\rho \rightarrow \infty$, uniformly in $\phi \in H^{1}\left(\mathbb{R}^{N}\right),\|\phi\|_{H^{1}\left(\mathbb{R}^{N}\right)} \leq C$; we use the same notation $\psi=\psi_{1,1}$ as in Step 5 above. Indeed, we denote $\widetilde{u}_{\rho}:=u-\psi$, so that $\widetilde{u}_{\rho} \rightarrow u$ strongly in $H^{1}\left(\mathbb{R}^{N}\right)$ as proved in Step 5 , and write

$$
\int_{\mathbb{R}^{N}}\left(\bar{f}\left(u_{\rho}-\widetilde{u}_{\rho}\right)-\bar{f}\left(u_{\rho}\right)\right) u_{\rho} \phi=I_{1}+I_{2}+I_{3},
$$

where

$$
\begin{aligned}
& I_{1}=\int_{\mathbb{R}^{N}}\left(f\left(u_{\rho}-u\right)-f\left(u_{\rho}\right)+f(u)\right) \phi ; \\
& I_{2}=\int_{\mathbb{R}^{N}}\left(f\left(u_{\rho}-\widetilde{u}_{\rho}\right)-f\left(u_{\rho}-u\right)\right) \phi ; \\
& I_{3}=\int_{\mathbb{R}^{N}}\left(\bar{f}\left(u_{\rho}-\widetilde{u}_{\rho}\right) \widetilde{u}_{\rho}-f(u)\right) \phi .
\end{aligned}
$$

Concerning the integral term $I_{1}$, we can apply the Cauchy-Schwarz inequality together with Lemma 5.2 to deduce that $I_{1} \rightarrow 0$ as $\rho \rightarrow \infty$, uniformly in bounded $\phi \in H^{1}\left(\mathbb{R}^{N}\right)$. The second integral term is handled trivially, since $f$ is $C^{1}$. As for $I_{3}$, for every $R>0$ let us denote $I_{3}^{R}=\int_{|x|<R}\left(\bar{f}\left(u_{\rho}-\widetilde{u}_{\rho}\right) \widetilde{u}_{\rho}-f(u)\right) \phi$. By the Hölder inequality we have

$$
\left|I_{3}^{R}\right|^{(r+1) / r} \leq C \int_{|x|<R}\left|\bar{f}\left(u_{\rho}-\widetilde{u}_{\rho}\right) \widetilde{u}_{\rho}-f(u)\right|^{(r+1) / r} .
$$


Using the Lebesgue dominated convergence theorem we see that $\left|I_{3}^{R}\right| \rightarrow 0$ as $\rho \rightarrow \infty$, for every $R>0$. On the other hand,

$$
\left|I_{3}-I_{3}^{R}\right| \leq C \int_{|x|>R}\left(|\phi|+|\phi|\left|u_{\rho}-\widetilde{u}_{\rho}\right|^{r-1}\right)\left|\widetilde{u}_{\rho}\right|+C \int_{|x|>R}\left(|\phi|+|\phi||u|^{r-1}\right)|u| \rightarrow 0
$$

as $R \rightarrow \infty$, uniformly in $\rho$ and in bounded $\phi \in H^{1}\left(\mathbb{R}^{N}\right)$. This shows that $I_{3} \rightarrow 0$ as $\rho \rightarrow \infty$, uniformly in bounded $\phi \in H^{1}\left(\mathbb{R}^{N}\right)$, and establishes our claim.

Step 7. We prove the estimate in (ii). By a direct computation, it amounts to proving that

$$
\limsup _{\rho \rightarrow \infty} I^{\prime \prime}\left(u_{\rho}-u+\psi, v_{\rho}-v-\psi\right)\left(u_{\rho}+\phi, v_{\rho}-\phi\right)\left(u_{\rho}+\phi, v_{\rho}-\phi\right)<0,
$$

uniformly in $\phi \in H^{1}\left(\mathbb{R}^{N}\right)$; we use the same notation $\psi=\psi_{1,1}$ as before. The expression on the left-hand side above is given by

$$
-\|\phi\|_{H^{1}\left(\mathbb{R}^{N}\right)}^{2}+\int_{\mathbb{R}^{N}} f\left(u_{\rho}\right) u_{\rho}+2 \int_{\mathbb{R}^{N}} f\left(u_{\rho}\right) \phi-\int_{\mathbb{R}^{N}} f^{\prime}\left(u_{\rho}-u+\psi\right)\left(u_{\rho}+\phi\right)^{2},
$$

plus a similar term related to the function $g$. Since $f^{\prime}(s) \geq\left(1+\delta^{\prime}\right) \bar{f}(s) \geq 0$ (cf. $(f g 3))$, this expression is bounded from above by

$$
\begin{aligned}
-\|\phi\|_{H^{1}\left(\mathbb{R}^{N}\right)}^{2} & +\int_{\mathbb{R}^{N}} f\left(u_{\rho}\right) u_{\rho}+2 \int_{\mathbb{R}^{N}} f\left(u_{\rho}\right) \phi \\
& -\int_{\mathbb{R}^{N}} \bar{f}\left(u_{\rho}-u+\psi\right)\left(u_{\rho}^{2}+2 u_{\rho} \phi\right)-\delta^{\prime} \int_{\mathbb{R}^{N}} \bar{f}\left(u_{\rho}-u+\psi\right)\left(u_{\rho}+\phi\right)^{2} .
\end{aligned}
$$

Thanks to the conclusion in Step 6, we can write this as

$$
-\|\phi\|_{H^{1}\left(\mathbb{R}^{N}\right)}^{2}-\delta^{\prime} \int_{\mathbb{R}^{N}} \bar{f}\left(u_{\rho}-u+\psi\right)\left(u_{\rho}+\phi\right)^{2}+\mathrm{o}(1) .
$$

In particular, we may already assume that $\|\phi\|_{H^{1}\left(\mathbb{R}^{N}\right)}=\mathrm{o}(1)$ as $\rho \rightarrow \infty$. But, in this case, thanks again to Step 6 , if $\rho$ is sufficiently large,

$$
\begin{aligned}
\delta^{\prime} \int_{\mathbb{R}^{N}} \bar{f}\left(u_{\rho}-u+\psi\right)\left(u_{\rho}+\phi\right)^{2} & \geq \delta^{\prime} \int_{\mathbb{R}^{N}} \bar{f}\left(u_{\rho}-u+\psi\right)\left(u_{\rho}^{2}+2 u_{\rho} \phi\right) \\
& =\mathrm{o}(1)+\delta^{\prime} \int_{\mathbb{R}^{N}} \bar{f}\left(u_{\rho}-u+\psi\right) u_{\rho}^{2} \\
& =\mathrm{o}(1)+\delta^{\prime} \int_{\mathbb{R}^{N}} f\left(u_{\rho}\right) u_{\rho},
\end{aligned}
$$

with

$\int_{\mathbb{R}^{N}} f\left(u_{\rho}\right) u_{\rho}=\int_{\mathbb{R}^{N}} f\left(u_{1, \rho}+u_{2, \rho}\right)\left(u_{1, \rho}+u_{2, \rho}\right) \geq \int_{\mathbb{R}^{N}} f\left(u_{1, \rho}\right) u_{1, \rho}=\int_{\mathbb{R}^{N}} f(u) u>0$.

Step 8 . The estimate in (iii) can be proved in a similar way as for (ii). One deduces now that

$$
\limsup _{\rho \rightarrow \infty} I^{\prime \prime}\left(u_{\rho}-u+\psi, v_{\rho}-v-\psi\right)(u+\phi, v-\phi)(u+\phi, v-\phi)<0,
$$

uniformly in $\phi \in H^{1}\left(\mathbb{R}^{N}\right)$, by showing that the following expression is negative for large values of $\rho$ :

$$
-\|\phi\|_{H^{1}\left(\mathbb{R}^{N}\right)}^{2}+\int_{\mathbb{R}^{N}} f(u) u+2 \int_{\mathbb{R}^{N}} f(u) \phi-\int_{\mathbb{R}^{N}} f^{\prime}\left(u_{\rho}-u+\psi\right)(u+\phi)^{2} .
$$


This, in turn, follows from the fact that, similarly to Step 6,

$$
\int_{\mathbb{R}^{N}} \bar{f}\left(u_{\rho}-u+\psi\right) u \phi=\int_{\mathbb{R}^{N}} \bar{f}(u) u \phi+o(1),
$$

as $\rho \rightarrow \infty$, uniformly in $\phi \in H^{1}\left(\mathbb{R}^{N}\right),\|\phi\|_{H^{1}\left(\mathbb{R}^{N}\right)} \leq C$.

Step 9. We prove the estimate in (iv). We must prove that

$$
\lim _{\rho \rightarrow \infty} I^{\prime \prime}\left(u_{\rho}-u+\psi, v_{\rho}-v-\psi\right)\left(u_{\rho}+\phi, v_{\rho}-\phi\right)(u, v)=0,
$$

where $\phi=\phi_{\rho}$ is the unique solution of the following equation in $H^{1}\left(\mathbb{R}^{N}\right)$ :

$$
\begin{aligned}
&-2 \Delta \phi+2 \phi+\left(f^{\prime}\left(u_{\rho}-u+\psi\right)+g^{\prime}\left(v_{\rho}-v-\psi\right)\right) \phi \\
& \quad f\left(u_{\rho}\right)-f^{\prime}\left(u_{\rho}-u+\psi\right) u_{\rho}+g^{\prime}\left(v_{\rho}-v-\psi\right) v_{\rho}-g\left(v_{\rho}\right) .
\end{aligned}
$$

We point out that $\phi \rightarrow 0$ weakly in $H^{1}\left(\mathbb{R}^{N}\right)$ (but not strongly). In particular, the above limit is given by

$$
\mathrm{o}(1)-\int_{\mathbb{R}^{N}} f^{\prime}\left(u_{\rho}-u+\psi\right)\left(u_{\rho}+\phi\right) u-\int_{\mathbb{R}^{N}} g^{\prime}\left(v_{\rho}-v-\psi\right)\left(v_{\rho}-\phi\right) v .
$$

Since $\psi \rightarrow 0$ strongly in $H^{1}\left(\mathbb{R}^{N}\right)$ and $u_{\rho}+\phi \rightarrow 0$ weakly in $H^{1}\left(\mathbb{R}^{N}\right)$, we have that

$$
\int_{\mathbb{R}^{N}} f^{\prime}\left(u_{\rho}-u+\psi\right)\left(u_{\rho}+\phi\right) u+\int_{\mathbb{R}^{N}} g^{\prime}\left(v_{\rho}-v-\psi\right)\left(v_{\rho}-\phi\right) v=\mathrm{o}(1) .
$$

This establishes (iv) and completes the argument.

\section{ACKNOWLEDGMENT}

We thank the referee for valuable comments that helped us to improve the first version of this manuscript.

The first author is thankful for the warm hospitality at IMECC-UNICAMP where part of this work was done.

The second author is thankful for the warm hospitality at the Laboratoire Jacques-Louis Lions, at the Département de Mathématique of ULB and at IMECCUNICAMP, where part of this work was done.

\section{REFERENCES}

1. C.O. Alves, P.C. Carrião, O.H. Miyagaki, On the existence of positive solutions of a perturbed Hamiltonian system in $\mathbb{R}^{N}$. J. Math. Anal. Appl. 276 (2002), no. 2, 673-690. MR1944783 (2003m:35051)

2. A. Alvino, P.-L. Lions, S. Matarasso, G. Trombetti, Comparison results for solutions of elliptic problems via symmetrization. Ann. Inst. H. Poincaré Anal. Non Linéaire 16 (1999), 167-188. MR 1674768 (2000b:35042)

3. A. Alvino, P.-L. Lions, G. Trombetti, A remark on comparison results via symmetrization. Proc. Roy. Soc. Edinburgh Sect. A 102 (1986), 37-48. MR837159 (88c:35007)

4. V. Benci, G. Cerami, Positive solutions of semilinear elliptic problems in exterior domains. Arch. Rat. Mech. Anal. 99 (1987), 283-300. MR898712 (88f:35014)

5. D. Bonheure, M. Ramos, Multiple critical points of perturbed symmetric strongly indefinite functionals. Ann. Inst. H. Poincaré Anal. Non Linéaire 26 (2009), 675-688. See also Erratum Ann. Inst. H. Poincaré Anal. Non Linéaire 26 (2009), 1049-1054. MR2504048 (2010f:35070) MR 2526415 (2010g:35077)

6. J. Busca, B. Sirakov, Symmetry results for semilinear elliptic systems in the whole space. J. Differential Equations 163 (2000), 41-56. MR1755067 (2001m:35100)

7. G. Cerami, M. Clapp, Sign changing solutions of semilinear elliptic problems in exterior domains. Calc. Var. Partial Differential Equations 31 (2008), 1-25. MR2332418(2008k:35138) 
8. G. Chiti, Rearrangements of functions and convergence in Orlicz spaces. Applicable Anal. 9 (1979), 23-27. MR536688 (80e:46020)

9. P. Clément, D.G. de Figueiredo, E. Mitidieri, Positive solutions of semilinear elliptic systems. Comm. Partial Differential Equations 17 (1992), 923-940. MR1177298 (93i:35054)

10. P. Clément, E. Mitidieri, On a class of nonlinear elliptic systems. Nonlinear evolution equations and their applications (Japanese) (Kyoto, 1996). Research Institute for Mathematical Sciences No. 1009 (1997), 132-140. MR.1630387 (99c:35055)

11. P. Clément, P. Felmer, E. Mitidieri, Homoclinic orbits for a class of infinite-dimensional Hamiltonian systems. Ann. Scuola Norm. Sup. Pisa Cl. Sci. (4) 24 (1997), no. 2, 367-393. MR.1487960 (99d:58036)

12. P. Clément, R. C. A. M. Van der Vorst, On a semilinear elliptic system. Differential Integral Equations 8 (1995), no. 6, 1317-1329. MR1329843 (96e:35043)

13. R. Dalmasso, Existence and uniqueness of positive solutions of semilinear elliptic systems. Nonlinear Analysis 39 (2000), 559-568. MR1727272 (2001d:35062)

14. R. Dalmasso, Existence and uniqueness of positive radial solutions for the Lane-Emden system. Nonlinear Analysis 57 (2004), 341-348. MR2064095 (2005b:35093)

15. D.G. de Figueiredo, Semilinear elliptic systems: Existence, multiplicity, symmetry of solutions. Handbook of Differential Equations: Stationary Partial Differential Equations, Volume 5, ed. M. Chipot, Elsevier (2008), 1-48. MR2497896 (2010j:35126)

16. D.G. de Figueiredo, P.L. Felmer, On superquadratic elliptic systems. Trans. Amer. Math. Soc. 343 (1994), 99-116. MR1214781 (94g:35072)

17. D.G. de Figueiredo, J. Yang, Decay, symmetry and existence of solutions of semilinear elliptic systems. Nonlinear Anal. TMA 33 (1998), 211-234. MR1617988 (99d:35047)

18. J. M. do Ó, P. Ubilla, A multiplicity result for a class of superquadratic Hamiltonian systems. Electron. J. Differential Equations 15 (2003), 14 pp. MR1958050 (2003k:35054)

19. E.M. dos Santos, Multiplicity of solutions for a fourth-order quasilinear nonhomogeneous equation. J. Math. Anal. Appl. 342 (2008), 277-297. MR2440796 (2010b:35091)

20. A. Ferrero, F. Gazzola, T. Weth, Positivity, symmetry and uniqueness for minimizers of second-order Sobolev inequalities. Ann. Mat. Pura Appl. (4) 186 (2007), 565-578. MR.2317778 (2009f:35057)

21. D. Gilbarg, N.S. Trudinger, "Elliptic partial differential equations of second order". Springer, 1977. MR0473443 (57:13109)

22. J. Hulshof, R.C.A.M. van der Vorst, Differential systems with strongly indefinite variational structure. J. Funct. Anal. 114 (1993), 32-58. MR.1220982 (94g:35073)

23. J. Hulshof, R.C.A.M. van der Vorst, Asymptotic behaviour of ground states. Proc. Amer. Math. Soc. 124 (1996), 2423-2431. MR1363170 (96m:35082)

24. P.L. Lions, The concentration-compactness principle in the calculus of variations, part 1. Rev. Mat. Iberoam. 1 (1985), 145-201. MR834360 (87c:49007)

25. E. Mitidieri, A Rellich type identity and applications. Comm. Partial Differential Equations 18 (1993), 125-151. MR:1211727 (94c:26016)

26. E. Mitidieri, Nonexistence of positive solutions of semilinear elliptic systems in $R^{N}$. Differential Integral Equations 9 (1996), no. 3, 465-479. MR.1371702 (96m:35087)

27. L.A. Peletier, R.C.A.M. van der Vorst, Existence and nonexistence of positive solutions of nonlinear elliptic systems and the biharmonic equation. Differential Integral Equations 5 (1992), 747-767. MR1167492 (93c:35039)

28. A. Pistoia, M. Ramos, Locating the peaks of the least energy solutions to an elliptic system with Dirichlet boundary conditions. NoDEA-Nonlinear Differential Equations Appl. 15 (2008), 1-23. MR.2408342 (2009b:35081)

29. M. Ramos, S. Soares, On the concentration of solutions of singularly perturbed Hamiltonian systems in $R^{N}$. Port. Math. (N.S.) 63 (2006), 157-171. MR2229874(2007f:35060)

30. M. Ramos, H. Tavares, Solutions with multiple spike patterns for an elliptic system. Calc. Var. Partial Differential Equations 31 (2008), 1-25. MR2342612 (2009c:35089)

31. M. Ramos, H. Tavares, W. Zou, A Bahri-Lions theorem revisited. Adv. Math. 222 (2009), 2173-2195. MR2562780

32. B. Ruf, Superlinear elliptic equations and systems, Handbook of Differential Equations: Stationary Partial Differential Equations, Volume 5, ed. M. Chipot, Elsevier (2008), 211-276. MR2497908 (2010d:35107) 
33. B. Sirakov, On the existence of solutions of Hamiltonian elliptic systems in $\mathbb{R}^{N}$. Adv. Differential Equations 5 (2000), 1445-1464. MR1785681 (2001g:35083)

34. G. Talenti, Elliptic equations and rearrangements. Ann. Scuola Norm. Sup. Pisa Cl. Sci. (4) (1976), 697-718. MR0601601 (58:29170)

35. W. C. Troy, Symmetry properties in systems of semilinear elliptic equations. J. Differential Equations 42 (1981), no. 3, 400-413. MR639230 (83b:35051)

36. R.C.A.M. van der Vorst, Best constant for the embedding of the space $H^{2} \cap H_{0}^{1}(\Omega)$ into $L^{2 N /(N-4)}(\Omega)$. Differential Integral Equations 6 (1993), no. 2, 259-276. MR.1195382 (94b:46053)

37. R.C.A.M. van der Vorst, Variational identities and applications to differential systems. Arch. Rational Mech. Anal. 116 (1991), 375-398. MR1132768 (93d:35043)

38. X. J. Wang, Sharp constant in a Sobolev inequality. Nonlinear Anal. 20 (1993), 261-268. MR.1202203 (94g:35035)

39. M. Willem, "Minimax theorems". Progress in Nonlinear Differential Equations and their Applications 24, Birkhäuser, Boston-Basel-Berlin, 1996. MR1400007 (97h:58037)

40. M. Willem, "Principes d'Analyse Fonctionnelle". Cassini, Paris, 2007. MR 2567317 (2010g:46001)

Département de Mathématique, Université libre de Bruxelles, CP 214, Boulevard du Triomphe, B-1050 Bruxelles, Belgium

E-mail address: denis.bonheure@ulb.ac.be

Instituto de Ciências Matemáticas e de Computaçäo, Universidade de São Paulo, Caixa Postal 668, CeP 13560-970, SÃo Carlos - SP, Brazil

E-mail address: ederson@icmc.usp.br

Faculty of Science, CMaf, University of Lisbon, Av. Prof. Gama Pinto 2, 1649-003 Lisboa, Portugal

E-mail address: mramos@ptmat.fc.ul.pt 